\title{
High-density Circumstellar Interaction in the Luminous Type lin Sn 2010jl: The First 1100 Days
}

\section{Citation}

Fransson, Claes, Mattias Ergon, Peter J. Challis, Roger A. Chevalier, Kevin France, Robert P. Kirshner, G. H. Marion, et al. 2014. "HIGH-DENSITY CIRCUMSTELLAR INTERACTION IN THE LUMINOUS TYPE IIn SN 2010jl: THE FIRST 1100 DAYS." The Astrophysical Journal 797 (2): 118. https://doi.org/10.1088/0004-637x/797/2/118.

\section{Permanent link}

http://nrs.harvard.edu/urn-3:HUL.InstRepos:41399808

\section{Terms of Use}

This article was downloaded from Harvard University's DASH repository, and is made available under the terms and conditions applicable to Other Posted Material, as set forth at http:// nrs.harvard.edu/urn-3:HUL.InstRepos:dash.current.terms-of-use\#LAA

\section{Share Your Story}

The Harvard community has made this article openly available. Please share how this access benefits you. Submit a story.

Accessibility 


\title{
HIGH-DENSITY CIRCUMSTELLAR INTERACTION IN THE LUMINOUS TYPE IIn SN 2010j1: THE FIRST 1100 DAYS
}

\author{
Clates Fransson $^{1}$, Mattias Ergon $^{1}$, Peter J. Challis ${ }^{2}$, Roger A. Chevalier ${ }^{3}$, Kevin France $^{4}$, Robert P. Kirshner ${ }^{2}$, \\ G. H. Marion ${ }^{2}$, Dan Milisavljevic ${ }^{2}$, Nathan Smith ${ }^{5}$, Filomena Bufano ${ }^{6}$, Andrew S. Friedman ${ }^{2}$, Tuomas Kangas ${ }^{7,8}$, \\ Josefin Larsson ${ }^{9}$, Seppo Mattila ${ }^{10}$, Stefano Benetti ${ }^{11}$, Ryan Chornock ${ }^{2}$, Ian Czekala ${ }^{2}$, \\ Alicia Soderberg ${ }^{2}$, And Jesper Sollerman ${ }^{1}$ \\ ${ }^{1}$ Oskar Klein Centre, Department of Astronomy, Stockholm University, AlbaNova, SE-106 91 Stockholm, Sweden \\ ${ }^{2}$ Harvard-Smithsonian Center for Astrophysics, 60 Garden Street, Cambridge, MA 02138, USA \\ ${ }^{3}$ Department of Astronomy, University of Virginia, P.O. Box 400325, Charlottesville, VA 22904, USA \\ ${ }^{4}$ CASA, University of Colorado, 593UCB Boulder, CO 80309-0593, USA \\ ${ }^{5}$ Steward Observatory, University of Arizona, 933 North Cherry Avenue, Tucson, AZ 85721, USA \\ ${ }^{6}$ Departamento de Ciencias Fisicas, Universidad Andres Bello, Avda. Republica 252, Santiago, Chile \\ ${ }^{7}$ Tuorla Observatory, University of Turku, Väisäläntie 20 FI-21500 Piikkiö, Finland \\ ${ }^{8}$ Nordic Optical Telescope (NOT), Apartado 474, E-38700 Santa Cruz de La Palma, Spain \\ ${ }^{9}$ KTH, Department of Physics, and the Oskar Klein Centre, AlbaNova, SE-106 91 Stockholm, Sweden \\ ${ }^{10}$ Finnish Centre for Astronomy with ESO (FINCA), University of Turku, Väisäläntie 20 FI-21500 Piikkiö, Finland \\ ${ }^{11}$ INAF-Osservatorio Astronomico di Padova, Vicolo dellOsservatorio 5, I-35122 Padova, Italy \\ Received 2014 January 10; accepted 2014 October 7; published 2014 December 8
}

\begin{abstract}
Hubble Space Telescope and ground-based observations of the Type IIn supernova (SN) 2010j1 are analyzed, including photometry and spectroscopy in the ultraviolet, optical, and near-IR bands, 26-1128 days after first detection. At maximum, the bolometric luminosity was $\sim 3 \times 10^{43} \mathrm{erg} \mathrm{s}^{-1}$ and even at 850 days exceeds $10^{42} \mathrm{erg} \mathrm{s}^{-1}$. A near-IR excess, dominating after 400 days, probably originates in dust in the circumstellar medium (CSM). The total radiated energy is $\gtrsim 6.5 \times 10^{50} \mathrm{erg}$, excluding the dust component. The spectral lines can be separated into one broad component that is due to electron scattering and one narrow with expansion velocity $\sim 100 \mathrm{~km} \mathrm{~s}^{-1}$ from the CSM. The broad component is initially symmetric around zero velocity but becomes blueshifted after $\sim 50$ days, while remaining symmetric about a shifted centroid velocity. Dust absorption in the ejecta is unlikely to explain the line shifts, and we attribute the shift instead to acceleration by the SN radiation. From the optical lines and the $\mathrm{X}$-ray and dust properties, there is strong evidence for large-scale asymmetries in the CSM. The ultraviolet lines indicate $\mathrm{CNO}$ processing in the progenitor, while the optical shows a number of narrow coronal lines excited by the X-rays. The bolometric light curve is consistent with a radiative shock in an $r^{-2}$ CSM with a mass-loss rate of $\dot{M} \sim 0.1 \quad M_{\odot} \mathrm{yr}^{-1}$. The total mass lost is $\gtrsim 3 M_{\odot}$. These properties are consistent with the SN expanding into a CSM characteristic of a luminous blue variable progenitor with a bipolar geometry. The apparent absence of nuclear processing is attributed to a CSM that is still opaque to electron scattering.
\end{abstract}

Key words: circumstellar matter - stars: mass-loss - supernovae: general - supernovae: individual (SN 2010jl)

Online-only material: color figures

\section{INTRODUCTION}

The progenitors for the narrow-lined Type II supernovae (SN IIn) remain a mystery, and this mystery is even deeper for the rare class of very luminous Type IIn with $M \lesssim-20$. Although significant evidence points toward an origin from luminous blue variable (LBV) stars (e.g., Smith 2008; Smith et al. 2008a, 2010a), suggestions for the energy source of the events span a wide range, from pair instabilities (Smith et al. 2007; Gal-Yam et al. 2009), magnetar power (Kasen \& Bildsten 2010; Nicholl et al. 2013), or extremely energetic core-collapse SNe (Umeda \& Nomoto 2008), to ongoing shock interactions or shock breakout from an extended progenitor (Falk \& Arnett 1977; Grasberg \& Nadyozhin 1987; Smith \& McCray 2007; Chevalier \& Irwin 2011; Ginzburg \& Balberg 2012).

Before SN 2010jl, the most convincing direct detection of a Type IIn progenitor was SN $2005 \mathrm{gl}$ at a distance of $66 \mathrm{Mpc}$, which was a moderately luminous SN IIn that transitioned into a more normal SN II. Preexplosion images of SN 2005gl showed a star at the position of the SN with a luminosity consistent with an LBV (Gal-Yam \& Leonard 2009). Recently it has been suggested that SN 1961V was in fact a core-collapse SN IIn, rather than an Eta Carinae-like eruption, in part because the peak absolute magnitude was -18 and because there is no surviving star detected in Spitzer images (Kochanek et al. 2011; Smith et al. 2011b). The progenitor of SN $1961 \mathrm{~V}$ was clearly detected for decades before 1961 as a very luminous blue star, consistent with an extremely massive LBV. Even more recently, SN 2009ip (Mauerhan et al. 2013; Pastorello et al. 2013; Fraser et al. 2013) and SN 2010mc (Ofek et al. 2013) have become interesting cases of a "supernova impostor" becoming a Type IIn SN. As we will show, these objects are similar in some ways, but differ from SN 2010jl in the essential feature of total energy release.

From a Spitzer/Infrared Array Camera survey of Type IIn $\mathrm{SNe}$, Fox et al. (2011) find mid-IR emission from $\sim 15 \%$ of the $\mathrm{SNe}$. The most likely origin is emission from preexisting dust heated by the optical emission from circumstellar interaction between the SN and the circumstellar medium (CSM) of the progenitor. Estimates of the dust mass are in the range $10^{-5}-10^{-1} M_{\odot}$. With a dust-to-gas ratio of $1: 100$, this is consistent with the total mass lost in giant LBV eruptions (Smith \& Owocki 2006).

While medium-luminosity Type IIn SNe, like SN $1988 Z$ and SN 1995N, often are strong radio and X-ray emitters, the 
high-luminosity IIn SNe are generally very weak. This may be a result of the extended massive envelopes thought to be responsible for the high luminosity of these $\mathrm{SNe}$, as discussed by Chevalier \& Irwin (2012).

In this paper we discuss Hubble Space Telescope (HST) and ground-based observations of the Type IIn SN 2010j1. SN 2010j1 was discovered on 2010 November 3.52 by Newton \& Puckett (2010). The first detection of SN 2010jl was, however, from prediscovery images on 2010 October 9.6 by Stoll et al. (2011). Based on the presence of narrow Balmer lines, it was classified as a Type IIn SN by Benetti et al. (2010).

From archival HST images, Smith et al. (2011a) identify a possible progenitor. Although the exact nature of this star is uncertain, several facts indicate a progenitor with mass $\gtrsim 30 M_{\odot}$, but it is difficult to distinguish between a single massive star or a young cluster. Stoll et al. (2011) find that the host is a lowmetallicity galaxy, as found for other luminous Type IIn host galaxies, which typically have metallicities of $\lesssim 0.4 Z_{\odot}$ (Neill et al. 2011; Chatzopoulos et al. 2011).

The peak $V$ and $I$ magnitudes were 13.7 and 13.0, respectively corresponding to absolute magnitudes $M_{V} \approx-19.9$ and $M_{I} \approx$ -20.5 (Stoll et al. 2011). This is considerably more luminous than the average Type IIn SN, $M_{V}=-18.4$ (Kiewe et al. 2012), and places it among the very luminous Type IIn SNe and close to the superluminous $\mathrm{SNe}$, defined as $M \lesssim-21$ (Gal-Yam 2012). The light curve showed a slow evolution with a decline by $\sim 1$ mag during the first 200 days (Andrews et al. 2011).

Smith et al. (2012b) report optical spectroscopy of the SN from 2 to 236 days after discovery. The spectrum shows a number of strong emission lines, with a narrow and a broad component. The $\mathrm{H} \alpha$ line is well fitted with a Lorentzian profile with a FWHM of $\sim 1800 \mathrm{~km} \mathrm{~s}^{-1}$ that extends to $\sim 6000 \mathrm{~km} \mathrm{~s}^{-1}$. The narrow $\mathrm{H} \alpha$ line was found to be double peaked and can be approximated by a Gaussian emission component with $\mathrm{FWHM}=120 \mathrm{~km} \mathrm{~s}^{-1}$. The shift of the line peak of the broad $\mathrm{H} \alpha$ line was seen as evidence for dust formation in the postshock gas. However, Smith et al. also discussed other scenarios based on a combination of electron scattering and continuum absorption. In this paper we show that electron scattering can explain all aspects of the line shapes.

Zhang et al. (2012) presented an extensive data set, including UBVRI photometry and low-resolution spectroscopy during the first $\sim 525$ days after first detection. Although the light curves and the optical low-resolution spectra agree well up to $\sim 200$ days, there are some important differences in the later observations and interpretation between Zhang et al. and this paper (see Section 3.2).

From Spitzer 3.6 and $4.5 \mu \mathrm{m}$ and $J H K$ photometry at an age of 90-108 days, Andrews et al. (2011) find evidence for preexisting dust with a mass of $0.03-0.35 M_{\odot}$ at a distance of $\sim 6 \times 10^{17} \mathrm{~cm}$ from the SN. Here, we complement these observations with more extensive coverage of the very late phases. An important clue to the geometry is that Patat et al. (2011) find strong, wavelength-independent polarization at a level of $\sim 2 \%$, which indicates an asphericity with axial ratio $\sim 0.7$.

SN 2010j1 was discovered as an X-ray source by Immler et al. (2010). Subsequent Chandra observations by Chandra et al. (2012b) on 2010 December 7-8, and 2011 October 17, i.e., 59 days and 373 days after first detection, indicate a hard spectrum with $k T \gtrsim 10 \mathrm{keV}$ and a very large column density at the first epoch, $N_{\mathrm{H}} \sim 10^{24} \mathrm{~cm}^{-2}$, which decreased to $N_{\mathrm{H}} \sim 3 \times 10^{23} \mathrm{~cm}^{-2}$ at 373 days. This absorption is most likely intrinsic to the SN or its CSM. NUSTAR and XMM observations by Ofek et al. (2014) in 2012 October to November (days 728-754) were able to provide a tighter constraint on the X-ray temperature of $\sim 12 \mathrm{keV}$. In addition, they also present UV and $\mathrm{X}$-ray observations with the $S W I F T$ satellite. No radio detections have been reported.

Ofek et al. (2014) also discussed the optical light curve and found a mass-loss rate of $\sim 0.8 M_{\odot} \mathrm{yr}^{-1}$, based on their assumed wind velocity of $300 \mathrm{~km} \mathrm{~s}^{-1}$ and a total mass lost of $\gtrsim 10 M_{\odot}$. Because of their limited optical photometry, they assumed a constant bolometric correction to the photometry. They also obtained spectroscopy at only a few epochs, and these data are most important at very late phases. While most of the qualitative conclusions in their paper are similar to ours, there are some important differences, which we discuss in the paper. They do not analyze the emission line spectrum in any detail, partly because of the lower spectral resolution of their data, which prevents separation of the narrow component from the broad.

Recently, Borish et al. (2014) discuss near-IR spectroscopic observations where they find evidence for high-velocity gas from the He I $\lambda 10,830$ line with velocities up to $5500 \mathrm{~km} \mathrm{~s}^{-1}$. This gas may be related to the X-ray-emitting gas, which originates from shocks with similar velocities.

Based on a single spectrum at 513 days, Maeda et al. (2013) proposed that the line shifts of the Balmer lines, discussed by Smith et al. (2012b) and in this paper, result from dust formation in the ejecta. This is also the subject of a paper by Gall et al. (2014), which makes a detailed analysis of the dust properties based on this interpretation. In this paper, based on extensive observations, we show that this interpretation, however, has severe problems.

In this paper we discuss an extensive set of optical, UV, and IR photometric and spectroscopic observations of SN 2010jl, together with interpretation of these observations. Compared to other papers, our data set includes broader wavelength coverage, more extensive time coverage, and higher spectral resolution. We present a unique set of UV observations obtained with HST. The X-ray observations and information about the progenitor from the literature enable us to provide a more complete picture of the event. In particular, we provide strong constraints on the structure of the progenitor and its environment.

Our paper is organized as follows. In Section 2 we report the observations, and we give our results in Section 3. In Section 4 we discuss these in relation to the properties of the CSM, dynamics, energetics, and different progenitor scenarios. We also put SN 2010j1 in the context of other IIn SNe. Our main conclusions are summarized in Section 5.

Based on the first detection, we adopt 2010 October 9 (Julian Date $2,455,479)$ as the explosion date of the $\mathrm{SN}$. With a recession velocity of the host galaxy of $3207 \pm 30 \mathrm{~km} \mathrm{~s}^{-1}$ (Stoll et al. 2011), we adopt a distance to UGC 5189A of $49 \pm 4 \mathrm{Mpc}$ in agreement with Smith et al. (2011a).

\section{OBSERVATIONS}

\subsection{Photometry}

Most of the optical imaging was obtained with the $1.2 \mathrm{~m}$ telescope at the F. L. Whipple Observatory (FLWO) using the KeplerCam instrument. KeplerCam data were reduced using the IRAF and IDL procedures described in Hicken et al. (2007). A few additional late-time epochs were obtained with the $6.5 \mathrm{~m}$ Baade telescope at the Magellan Observatory using the IMACS instrument and the $2.5 \mathrm{~m}$ Nordic Optical Telescope (NOT) using the ALFOSC instrument. These were reduced with IRAF 
using standard methods. High-quality NOT imaging was used to construct $B-, V-, r$-, and $i$-band templates by point-spread function (PSF) subtraction of the SN using the IRAFDAOPHOT package. These templates were then subtracted from the FLWO, Magellan Observatory, and NOT imaging using the HOTPANTS package. Template subtraction is necessary to obtain accurate photometry after $\sim 100$ days because the $\mathrm{SN}$ is located close to the center of the galaxy.

The optical photometry was calibrated to the JohnsonCousins (JC) and Sloan Digital Sky Survey (SDSS) standard systems using local reference stars in the $\mathrm{SN}$ field, in turn calibrated using standard field observations on five photometric nights. Because the SN spectral energy distribution (SED), in particular at late phases, is line dominated, we have applied the technique of $S$ corrections (Stritzinger et al. 2002) to transform from the natural system of each instrument to the standard systems. This was done for all bands except the $u$ band. Color terms and filter response functions were adopted from Hicken et al. (2012) for the FLWO and from Ergon et al. (2014) for the NOT. Filter response functions for the JC and SDSS standard systems were adopted from Bessell \& Murphy (2012) and Doi et al. (2010).

Most of the near-infrared (NIR) imaging was obtained with the $1.3 \mathrm{~m}$ Peters Automated Infrared Imaging Telescope (PAIRITEL) at the FLWO. The data were processed into mosaics using the PAIRITEL Mosaic Pipeline version 3.6 implemented in Python. Details of PAIRITEL observations and reduction of SN data can be found in Friedman et al. (2014). Two additional late-time epochs were obtained with NOT using the NOTCAM instrument. This high-quality imaging was used to construct $J$-, $H$-, and $K$-band templates by PSF subtraction of the SN using the IRAF DAOPHOT package. These templates were then subtracted from the PAIRITEL imaging using the HOTPANTS package. As in the optical, template subtraction is necessary to obtain accurate photometry after $\sim 100$ days and is particularly important for the late $J$-band photometry.

The NIR photometry was calibrated to the Two Micron All Sky Survey (2MASS) standard system using reference stars from the 2MASS Point Source catalog (Skrutskie et al. 2006) within the SN field. Because the PAIRITEL was one of the two telescopes used for the 2MASS survey, the natural system photometry is already on the 2MASS standard system, and no transformation is needed. The NOT photometry was transformed to the 2MASS standard system using linear color terms from Ergon et al. (2014). The absence of strong lines in the X-shooter NIR spectrum indicates that this is sufficient and that $S$ corrections are not needed.

As a check we can compare with the $J, H$, and $K_{s}$ magnitudes from Andrews et al. (2011) at 104 days. Our $J$ and $H$ magnitudes agree within $\sim 0.1 \mathrm{mag}$ with those of Andrews et al. However, our $K_{s}$ magnitude close to this epoch is $\sim 12.7$, while that of Andrews et al. is $\sim 13.75$. Judging from the SED plot in Andrews et al., it, however, seems that there is a typographical error and that the magnitude should be $12.75 \pm 0.1$. In this case there is excellent agreement.

In Tables 1 and 2 we give the photometry for all epochs, including errors.

\subsection{Spectroscopy}

\subsubsection{HST-COS and STIS Observations and Data Reduction}

HST observations with the Space Telescope Imaging Spectrograph (STIS) of SN2010jl were obtained at four epochs, 23, 33, 107, and 573 days after first detection. The observations were carried out as part of program GO-12242, UV Studies of a Core Collapse Supernova.The G230LB and G430L gratings were used at each epoch with the $52 \times 0$ '.2 slit. The data were reduced using the standard HST Space Telescope Science Data Analysis System routines to bias subtract, flat field, extract, wavelength calibrate, and flux calibrate each SN spectrum. The spectral resolution of these gratings corresponds to $500-600 \mathrm{~km} \mathrm{~s}^{-1}$ at the short wavelength limit of the gratings and $250-300 \mathrm{~km} \mathrm{~s}^{-1}$ at the long limit. Table 3 summarizes the STIS observations including exposure time and spectral resolution.

The SN was also observed with the medium-resolution, farUV modes of HST-COS (G130M and G160M) at 44 days, 107 days, and 621 days. A description of the COS instrument and on-orbit performance characteristics can be found in Osterman et al. (2011) and Green et al. (2012). All observations were centered on SN 2010j1 (R.A. $=09^{\mathrm{h}} 42^{\mathrm{m}} 53^{\mathrm{s}} .33$, Dec. $=$ $+09^{\circ} 29^{\prime} 41^{\prime \prime}$.8; J2000), and COS performed near-UV imaging target acquisitions with the PSA/MIRRORB mode. The MIRRORB configuration introduces optical distortions into the target acquisition image, but a first-order analysis indicates that the observations were centered on the point-like SN. It is important to realize that COS has only limited spatial resolution, which means that objects as far as $2^{\prime \prime}$ from the center contribute to the spectrum. This is important for objects with nearby $\mathrm{H}$ II regions. Our last COS spectrum of SN 2010jl, at 621 days, has strong galaxy contamination from this source.

The G130M data were processed with the COS calibration pipeline $\operatorname{CALCOS}^{12} \mathrm{v} 2.12$, and combined with the custom IDL coaddition procedure described by Danforth et al. (2010) and Shull et al. (2010). The coaddition routine interpolates all detector segments and grating settings onto a common wavelength grid and makes a correction for the detector QEenhancement grid. No correction for the detector hex pattern is performed. The data cover the 1155-1773 $\AA$ bandpass, with two breaks at the COS detector segment gaps, $\left[\lambda_{\text {gap }}, \Delta \lambda_{\text {gap }}\right]=$ [1302 $\AA, 16 \AA]$ and [1592 $\mathrm{A}, 20 \AA$ ], for the G130M and G160M modes, respectively. The resolving power of the mediumresolution COS modes is $R \equiv \lambda / \Delta \lambda \approx 18,000\left(\Delta v=17 \mathrm{~km} \mathrm{~s}^{-1}\right)$.

\subsubsection{Optical Spectroscopy}

Complementing the HST observations, an extensive spectroscopic campaign involving several ground-based telescopes was launched, and a log of all spectroscopic observations is available in Table 4.

Optical spectroscopy of SN 2010jl was obtained on 16 epochs with the $2.5 \mathrm{~m}$ NOT at La Palma, Spain, with the ALFOSC spectrograph. To optimize the resolution for this narrow-line $\mathrm{SN}$, we used two setups. Grism 17 covers basically only the $\mathrm{H} \alpha$ region, and grism 16 was used for the bluer part, covering the rest of the Balmer series and connecting with the HST near-UV (NUV) data. All observations were performed at the parallactic angle, and the air mass was always less than 1.4 at the start of the observations. To further enhance the resolution, we used narrow slits of width 0.9 arcsec for grism 17 and $0.5-0.75$ arcsec for grism 16. The spectra were reduced in a standard manner using IRAF scripts as implemented in the QUBA pipeline. Wavelength calibrations were determined from exposures of $\mathrm{HeNe}$ arc lamps and were checked against bright night sky emission lines. Flux calibration was performed by means of spectrophotometric standard stars.

\footnotetext{
12 We refer the reader to the Cycle 18 COS Instrument Handbook for more details:

http://www.stsci.edu/hst/cos/documents/handbooks/current/cos_cover.html.
} 
Table 1

Optical Photometry

\begin{tabular}{|c|c|c|c|c|c|c|c|}
\hline$\overline{\mathrm{JD}}$ & Epoch $^{\mathrm{a}}$ & $\overline{u^{\prime}}$ & $B$ & $\bar{V}$ & $r^{\prime}$ & $\overline{i^{\prime}}$ & $\begin{array}{c}\text { Telescope } \\
\text { (Instrument) }\end{array}$ \\
\hline 55508.98 & 29.98 & $13.85(0.12)$ & $14.08(0.03)$ & $13.79(0.03)$ & $13.49(0.02)$ & $13.54(0.03)$ & FLWO (KeplerCam) \\
\hline 55510.98 & 31.98 & $13.91(0.12)$ & $14.10(0.03)$ & $13.80(0.03)$ & $13.49(0.02)$ & $13.55(0.03)$ & FLWO (KeplerCam) \\
\hline 55513.96 & 34.96 & $13.94(0.12)$ & $14.13(0.03)$ & $13.84(0.03)$ & $13.52(0.02)$ & $13.58(0.03)$ & FLWO (KeplerCam) \\
\hline 55516.00 & 37.00 & $14.00(0.12)$ & $14.16(0.03)$ & $13.85(0.03)$ & $13.53(0.02)$ & $13.60(0.03)$ & FLWO (KeplerCam) \\
\hline 55522.91 & 43.91 & & & $13.88(0.03)$ & $13.55(0.02)$ & $13.63(0.03)$ & FLWO (KeplerCam) \\
\hline 55527.02 & 48.02 & $14.12(0.12)$ & $14.30(0.03)$ & $13.95(0.03)$ & $13.64(0.02)$ & $13.68(0.03)$ & FLWO (KeplerCam) \\
\hline 55529.95 & 50.95 & $14.19(0.12)$ & $14.35(0.03)$ & $14.02(0.03)$ & $13.66(0.02)$ & $13.74(0.03)$ & FLWO (KeplerCam) \\
\hline 55530.93 & 51.93 & $14.24(0.12)$ & $14.31(0.03)$ & $13.99(0.03)$ & $13.61(0.02)$ & $13.74(0.03)$ & FLWO (KeplerCam) \\
\hline 55533.98 & 54.98 & $14.22(0.12)$ & $14.38(0.03)$ & $14.07(0.03)$ & $13.71(0.02)$ & $13.79(0.03)$ & FLWO (KeplerCam) \\
\hline 55537.01 & 58.01 & $14.25(0.12)$ & $14.40(0.03)$ & $14.11(0.03)$ & $13.71(0.02)$ & $13.83(0.03)$ & FLWO (KeplerCam) \\
\hline 55540.95 & 61.95 & $14.30(0.12)$ & $14.39(0.03)$ & $14.09(0.03)$ & $13.69(0.02)$ & $13.82(0.03)$ & FLWO (KeplerCam) \\
\hline 55558.99 & 79.99 & $14.57(0.12)$ & $14.62(0.03)$ & $14.32(0.03)$ & $13.87(0.02)$ & $14.03(0.03)$ & FLWO (KeplerCam) \\
\hline 55564.02 & 85.02 & $14.54(0.12)$ & $14.77(0.03)$ & $14.46(0.03)$ & $13.89(0.02)$ & $14.13(0.03)$ & FLWO (KeplerCam) \\
\hline 55565.02 & 86.02 & $14.52(0.12)$ & $14.84(0.03)$ & $14.47(0.03)$ & $13.89(0.02)$ & $14.14(0.03)$ & FLWO (KeplerCam) \\
\hline 55566.03 & 87.03 & $14.61(0.12)$ & $14.82(0.03)$ & $14.51(0.03)$ & $13.91(0.02)$ & $14.18(0.03)$ & FLWO (KeplerCam) \\
\hline 55566.91 & 87.91 & $14.61(0.12)$ & $14.80(0.03)$ & $14.48(0.03)$ & $13.88(0.02)$ & $14.15(0.03)$ & FLWO (KeplerCam) \\
\hline 55568.98 & 89.98 & $14.64(0.12)$ & & $14.51(0.03)$ & $13.90(0.02)$ & $14.20(0.03)$ & FLWO (KeplerCam) \\
\hline 55570.00 & 91.00 & $14.62(0.12)$ & $14.81(0.03)$ & $14.50(0.03)$ & $13.91(0.02)$ & $14.20(0.03)$ & FLWO (KeplerCam) \\
\hline 55571.98 & 92.98 & $14.62(0.12)$ & $14.86(0.03)$ & $14.51(0.03)$ & $13.91(0.02)$ & $14.21(0.03)$ & FLWO (KeplerCam) \\
\hline 55572.86 & 93.86 & $14.63(0.12)$ & $14.84(0.03)$ & $14.54(0.03)$ & $13.92(0.02)$ & $14.21(0.03)$ & FLWO (KeplerCam) \\
\hline 55575.89 & 96.89 & $14.62(0.12)$ & $14.91(0.03)$ & $14.55(0.03)$ & $13.94(0.02)$ & $14.24(0.03)$ & FLWO (KeplerCam) \\
\hline 55576.76 & 97.76 & & & $14.65(0.03)$ & $13.94(0.02)$ & $14.28(0.03)$ & FLWO (KeplerCam) \\
\hline 55578.00 & 99.00 & $14.76(0.12)$ & $14.92(0.03)$ & $14.57(0.03)$ & $13.93(0.02)$ & $14.26(0.03)$ & FLWO (KeplerCam) \\
\hline 55580.88 & 101.88 & $14.66(0.12)$ & $\ldots$ & $14.62(0.03)$ & $13.94(0.02)$ & $14.27(0.03)$ & FLWO (KeplerCam) \\
\hline 55587.89 & 108.89 & $14.80(0.12)$ & & $14.62(0.03)$ & $13.95(0.02)$ & $14.34(0.03)$ & FLWO (KeplerCam) \\
\hline 55588.88 & 109.88 & & $14.94(0.03)$ & $14.63(0.03)$ & $13.96(0.02)$ & $14.33(0.03)$ & FLWO (KeplerCam) \\
\hline 55589.87 & 110.87 & $14.72(0.12)$ & $14.95(0.03)$ & $14.63(0.03)$ & $13.98(0.02)$ & $14.34(0.03)$ & FLWO (KeplerCam) \\
\hline 55594.91 & 115.91 & $14.71(0.12)$ & $15.00(0.03)$ & $14.70(0.03)$ & $13.97(0.02)$ & $14.37(0.03)$ & FLWO (KeplerCam) \\
\hline 55597.77 & 118.77 & & $14.98(0.03)$ & $14.69(0.03)$ & $13.99(0.02)$ & $14.39(0.03)$ & FLWO (KeplerCam) \\
\hline 55600.86 & 121.86 & $14.94(0.12)$ & $15.00(0.03)$ & $14.71(0.03)$ & $13.98(0.02)$ & $14.40(0.03)$ & FLWO (KeplerCam) \\
\hline 55602.70 & 123.70 & $14.76(0.12)$ & & $14.71(0.03)$ & $13.99(0.02)$ & $14.41(0.03)$ & FLWO (KeplerCam) \\
\hline 55605.94 & 126.94 & $14.79(0.12)$ & $15.09(0.03)$ & $14.75(0.03)$ & $14.01(0.02)$ & $14.44(0.03)$ & FLWO (KeplerCam) \\
\hline 55606.82 & 127.82 & $14.85(0.12)$ & & & & & FLWO (KeplerCam) \\
\hline 55607.93 & 128.93 & $14.88(0.12)$ & $15.08(0.03)$ & $14.77(0.03)$ & $14.02(0.02)$ & $14.44(0.03)$ & FLWO (KeplerCam) \\
\hline 55608.86 & 129.86 & $14.83(0.12)$ & $\ldots$ & $14.75(0.03)$ & $14.00(0.02)$ & $14.45(0.03)$ & FLWO (KeplerCam) \\
\hline 55615.95 & 136.95 & $14.81(0.12)$ & & $14.79(0.03)$ & $14.00(0.02)$ & $14.51(0.03)$ & FLWO (KeplerCam) \\
\hline 55623.65 & 144.65 & $14.82(0.12)$ & $15.13(0.03)$ & $14.81(0.03)$ & $14.02(0.02)$ & $14.53(0.03)$ & FLWO (KeplerCam) \\
\hline 55628.78 & 149.78 & $14.87(0.12)$ & $15.12(0.03)$ & $14.83(0.03)$ & & $14.56(0.03)$ & FLWO (KeplerCam) \\
\hline 55631.66 & 152.66 & $14.98(0.12)$ & $15.14(0.03)$ & $14.84(0.03)$ & $14.02(0.02)$ & $14.55(0.03)$ & FLWO (KeplerCam) \\
\hline 55647.73 & 168.73 & $15.02(0.12)$ & $15.17(0.03)$ & $14.90(0.03)$ & $14.02(0.02)$ & $14.63(0.03)$ & FLWO (KeplerCam) \\
\hline 55667.74 & 188.74 & $\ldots$ & $\ldots$ & $14.96(0.03)$ & $14.03(0.02)$ & $14.69(0.03)$ & FLWO (KeplerCam) \\
\hline 55668.81 & 189.81 & $\ldots$ & $\ldots$ & $14.98(0.03)$ & $14.03(0.02)$ & $14.69(0.03)$ & FLWO (KeplerCam) \\
\hline 55669.74 & 190.74 & $\ldots$ & & $14.97(0.03)$ & $14.03(0.02)$ & $14.69(0.03)$ & FLWO (KeplerCam) \\
\hline 55673.70 & 194.70 & $\ldots$ & $15.21(0.03)$ & $14.97(0.03)$ & $14.04(0.02)$ & $14.69(0.03)$ & FLWO (KeplerCam) \\
\hline 55682.68 & 203.68 & $\ldots$ & $15.23(0.03)$ & $15.00(0.03)$ & $14.04(0.02)$ & $14.72(0.03)$ & FLWO (KeplerCam) \\
\hline 55686.68 & 207.68 & $\ldots$ & $15.22(0.03)$ & & $14.03(0.02)$ & $14.73(0.03)$ & FLWO (KeplerCam) \\
\hline 55688.67 & 209.67 & $\ldots$ & $15.24(0.03)$ & $14.98(0.03)$ & $14.02(0.02)$ & $14.72(0.03)$ & FLWO (KeplerCam) \\
\hline 55691.70 & 212.70 & $\ldots$ & $15.21(0.03)$ & $15.02(0.03)$ & $14.04(0.02)$ & $14.76(0.03)$ & FLWO (KeplerCam) \\
\hline 55696.63 & 217.63 & $\ldots$ & $\ldots$ & $15.01(0.03)$ & $14.04(0.02)$ & $14.74(0.03)$ & FLWO (KeplerCam) \\
\hline 55698.67 & 219.67 & $\ldots$ & $\ldots$ & $15.04(0.03)$ & $14.04(0.02)$ & $14.76(0.03)$ & FLWO (KeplerCam) \\
\hline 55704.68 & 225.68 & $\ldots$ & & $15.01(0.03)$ & $14.04(0.02)$ & $14.78(0.03)$ & FLWO (KeplerCam) \\
\hline 55717.68 & 238.68 & $\ldots$ & $15.27(0.03)$ & $15.05(0.03)$ & $14.05(0.02)$ & $14.83(0.03)$ & FLWO (KeplerCam) \\
\hline 55855.99 & 376.99 & $\ldots$ & $16.38(0.03)$ & & $14.72(0.02)$ & $15.72(0.03)$ & FLWO (KeplerCam) \\
\hline 55866.97 & 387.97 & $\ldots$ & $16.39(0.03)$ & $16.15(0.03)$ & $14.79(0.02)$ & $15.79(0.03)$ & FLWO (KeplerCam) \\
\hline 55888.01 & 409.01 & $\ldots$ & $16.71(0.03)$ & $16.40(0.03)$ & $15.01(0.02)$ & $15.98(0.03)$ & FLWO (KeplerCam) \\
\hline 55888.98 & 409.98 & $\ldots$ & $16.70(0.03)$ & $16.43(0.03)$ & $15.02(0.02)$ & $15.98(0.03)$ & FLWO (KeplerCam) \\
\hline 55916.98 & 437.98 & $\ldots$ & $17.08(0.03)$ & $16.77(0.03)$ & $15.25(0.02)$ & $16.27(0.03)$ & FLWO (KeplerCam) \\
\hline 55918.82 & 439.82 & $\ldots$ & $17.20(0.03)$ & $16.82(0.03)$ & $15.29(0.02)$ & $16.27(0.03)$ & FLWO (KeplerCam) \\
\hline 55948.84 & 469.84 & $\ldots$ & $17.47(0.03)$ & $17.06(0.03)$ & $15.52(0.02)$ & $16.52(0.03)$ & FLWO (KeplerCam) \\
\hline 55952.80 & 473.80 & $\ldots$ & $17.41(0.03)$ & $17.03(0.03)$ & $15.50(0.02)$ & $16.57(0.03)$ & FLWO (KeplerCam) \\
\hline 55972.87 & 493.87 & $\ldots$ & $17.54(0.03)$ & $17.33(0.03)$ & $15.62(0.02)$ & $16.73(0.03)$ & FLWO (KeplerCam) \\
\hline 55978.77 & 499.77 & $\ldots$ & $17.62(0.03)$ & $17.26(0.03)$ & $15.65(0.02)$ & $16.71(0.03)$ & FLWO (KeplerCam) \\
\hline 56009.73 & 530.73 & $\ldots$ & $17.80(0.03)$ & $17.62(0.03)$ & $15.81(0.02)$ & $16.97(0.03)$ & FLWO (KeplerCam) \\
\hline 56037.66 & 558.66 & $\ldots$ & $18.16(0.03)$ & $17.83(0.03)$ & $15.95(0.02)$ & $17.23(0.03)$ & FLWO (KeplerCam) \\
\hline 56040.65 & 561.65 & $\ldots$ & $18.01(0.03)$ & $17.84(0.03)$ & $15.99(0.02)$ & & FLWO (KeplerCam) \\
\hline 56060.63 & 581.63 & $\ldots$ & $18.36(0.03)$ & $17.89(0.03)$ & $16.06(0.02)$ & $17.33(0.03)$ & FLWO (KeplerCam) \\
\hline 56065.63 & 586.63 & $\ldots$ & $18.29(0.03)$ & $18.02(0.03)$ & $16.12(0.02)$ & $17.48(0.03)$ & FLWO (KeplerCam) \\
\hline 56241.73 & 762.73 & $\ldots$ & $19.43(0.03)$ & $19.19(0.03)$ & $17.04(0.02)$ & $18.76(0.03)$ & NOT (ALFOSC) \\
\hline 56248.83 & 769.83 & $\ldots$ & $19.61(0.03)$ & $19.31(0.03)$ & $17.10(0.02)$ & $18.52(0.03)$ & MAG (IMACS) \\
\hline 56327.54 & 848.54 & $\ldots$ & $19.91(0.03)$ & $19.73(0.03)$ & $17.59(0.02)$ & & NOT (ALFOSC) \\
\hline 56396.48 & 917.48 & $19.16(0.12)$ & $20.43(0.03)$ & $20.40(0.03)$ & $18.19(0.02)$ & $20.14(0.03)$ & NOT (ALFOSC) \\
\hline
\end{tabular}

Note. ${ }^{a}$ Relative to first detection date, JD 2,455,479.0. 
Table 2

Near-infrared Photometry

\begin{tabular}{|c|c|c|c|c|c|}
\hline JD & Epoch & $J$ & $H$ & $K$ & Telescope (Instrument) \\
\hline 55505.94 & 26.94 & $12.84(0.06)$ & $12.56(0.07)$ & & PAIRITEL (2MASS) \\
\hline 55507.99 & 28.99 & $\ldots$ & $\ldots$ & $12.32(0.08)$ & PAIRITEL (2MASS) \\
\hline 55508.98 & 29.98 & $\ldots$ & $12.57(0.07)$ & $\ldots$ & PAIRITEL (2MASS) \\
\hline 55511.96 & 32.96 & $12.88(0.06)$ & $\ldots$ & $\ldots$ & PAIRITEL (2MASS) \\
\hline 55514.90 & 35.90 & $12.89(0.06)$ & $12.56(0.07)$ & $\ldots$ & PAIRITEL (2MASS) \\
\hline 55517.97 & 38.97 & $\ldots$ & $12.68(0.07)$ & $\ldots$ & PAIRITEL (2MASS) \\
\hline 55518.95 & 39.95 & $12.88(0.06)$ & $\ldots$ & $12.36(0.08)$ & PAIRITEL (2MASS) \\
\hline 55524.00 & 45.00 & $12.92(0.06)$ & $12.61(0.07)$ & $\ldots$ & PAIRITEL (2MASS) \\
\hline 55525.03 & 46.03 & $12.87(0.06)$ & $\ldots$ & $\ldots$ & PAIRITEL (2MASS) \\
\hline 55533.85 & 54.85 & $13.03(0.06)$ & $\ldots$ & $\ldots$ & PAIRITEL (2MASS) \\
\hline 55536.95 & 57.95 & $13.04(0.06)$ & $\ldots$ & $12.34(0.08)$ & PAIRITEL (2MASS) \\
\hline 55537.95 & 58.95 & $13.04(0.06)$ & $12.69(0.07)$ & $12.41(0.08)$ & PAIRITEL (2MASS) \\
\hline 55540.87 & 61.87 & $13.05(0.06)$ & $12.82(0.07)$ & $12.43(0.08)$ & PAIRITEL (2MASS) \\
\hline 55569.92 & 90.92 & $13.27(0.06)$ & $13.04(0.07)$ & $12.54(0.08)$ & PAIRITEL (2MASS) \\
\hline 55572.84 & 93.84 & $13.26(0.06)$ & $\ldots$ & $\ldots$ & PAIRITEL (2MASS) \\
\hline 55575.83 & 96.83 & $13.28(0.06)$ & $13.07(0.07)$ & $12.69(0.08)$ & PAIRITEL (2MASS) \\
\hline 55578.79 & 99.79 & $13.30(0.06)$ & $13.03(0.07)$ & $12.66(0.08)$ & PAIRITEL (2MASS) \\
\hline 55599.77 & 120.77 & $13.42(0.06)$ & $13.19(0.07)$ & $12.76(0.08)$ & PAIRITEL (2MASS) \\
\hline 55602.70 & 123.70 & $13.44(0.06)$ & $13.13(0.07)$ & $12.81(0.08)$ & PAIRITEL (2MASS) \\
\hline 55607.80 & 128.80 & $13.47(0.06)$ & $\ldots$ & $\ldots$ & PAIRITEL (2MASS) \\
\hline 55617.74 & 138.74 & & $13.25(0.07)$ & $12.86(0.08)$ & PAIRITEL (2MASS) \\
\hline 55620.76 & 141.76 & $13.53(0.06)$ & $\ldots$ & $12.86(0.08)$ & PAIRITEL (2MASS) \\
\hline 55623.77 & 144.77 & $\ldots$ & $13.29(0.07)$ & $\ldots$ & PAIRITEL (2MASS) \\
\hline 55629.73 & 150.73 & $13.55(0.06)$ & $\ldots$ & $12.84(0.08)$ & PAIRITEL (2MASS) \\
\hline 55640.73 & 161.73 & $13.63(0.06)$ & $13.31(0.07)$ & $\ldots$ & PAIRITEL (2MASS) \\
\hline 55643.73 & 164.73 & $13.59(0.06)$ & $\ldots$ & $12.91(0.08)$ & PAIRITEL (2MASS) \\
\hline 55644.71 & 165.71 & $13.57(0.06)$ & $13.32(0.07)$ & & PAIRITEL (2MASS) \\
\hline 55647.79 & 168.79 & $13.61(0.06)$ & $\ldots$ & $12.99(0.08)$ & PAIRITEL (2MASS) \\
\hline 55651.72 & 172.72 & $13.63(0.06)$ & $13.36(0.07)$ & $12.96(0.08)$ & PAIRITEL (2MASS) \\
\hline 55670.66 & 191.66 & $13.61(0.06)$ & $\ldots$ & $\ldots$ & PAIRITEL (2MASS) \\
\hline 55676.68 & 197.68 & $13.65(0.06)$ & $13.49(0.07)$ & $13.06(0.08)$ & PAIRITEL (2MASS) \\
\hline 55688.63 & 209.63 & $13.63(0.06)$ & $13.51(0.07)$ & $12.95(0.08)$ & PAIRITEL (2MASS) \\
\hline 55705.68 & 226.68 & $13.72(0.06)$ & $13.51(0.07)$ & $\ldots$ & PAIRITEL (2MASS) \\
\hline 55708.67 & 229.67 & $13.66(0.06)$ & $13.52(0.07)$ & $\ldots$ & PAIRITEL (2MASS) \\
\hline 55947.89 & 468.89 & $13.76(0.06)$ & $12.76(0.07)$ & $11.88(0.08)$ & PAIRITEL (2MASS) \\
\hline 55949.87 & 470.87 & $\ldots$ & $\ldots$ & $11.79(0.08)$ & PAIRITEL (2MASS) \\
\hline 55999.65 & 520.65 & $14.01(0.06)$ & $\ldots$ & $11.86(0.08)$ & PAIRITEL (2MASS) \\
\hline 56002.59 & 523.59 & $\ldots$ & $12.81(0.07)$ & $\ldots$ & PAIRITEL (2MASS) \\
\hline 56010.63 & 531.63 & $\ldots$ & $12.80(0.07)$ & $11.96(0.08)$ & PAIRITEL (2MASS) \\
\hline 56011.63 & 532.63 & $14.06(0.06)$ & $\ldots$ & $\ldots$ & PAIRITEL (2MASS) \\
\hline 56013.71 & 534.71 & $\ldots$ & $12.84(0.07)$ & $11.94(0.08)$ & PAIRITEL (2MASS) \\
\hline 56014.63 & 535.63 & $14.06(0.06)$ & $\ldots$ & $\ldots$ & PAIRITEL (2MASS) \\
\hline 56015.69 & 536.69 & $\ldots$ & $12.82(0.07)$ & $11.86(0.08)$ & PAIRITEL (2MASS) \\
\hline 56017.69 & 538.69 & $14.06(0.06)$ & $12.84(0.07)$ & . & PAIRITEL (2MASS) \\
\hline 56019.70 & 540.70 & $\ldots$ & $\ldots$ & $11.88(0.08)$ & PAIRITEL (2MASS) \\
\hline 56025.70 & 546.70 & $14.14(0.06)$ & $\ldots$ & $\ldots$ & PAIRITEL (2MASS) \\
\hline 56026.68 & 547.68 & $14.15(0.06)$ & $12.88(0.07)$ & $\ldots$ & PAIRITEL (2MASS) \\
\hline 56027.69 & 548.69 & $14.10(0.06)$ & $\ldots$ & $\ldots$ & PAIRITEL (2MASS) \\
\hline 56028.68 & 549.68 & $14.16(0.06)$ & $\ldots$ & $\ldots$ & PAIRITEL (2MASS) \\
\hline 56029.68 & 550.68 & $14.16(0.06)$ & $\ldots$ & $11.95(0.08)$ & PAIRITEL (2MASS) \\
\hline 56031.68 & 552.68 & $14.16(0.06)$ & $\ldots$ & $12.03(0.08)$ & PAIRITEL (2MASS) \\
\hline 56035.61 & 556.61 & $\ldots$ & $12.88(0.07)$ & $12.02(0.08)$ & PAIRITEL (2MASS) \\
\hline 56036.68 & 557.68 & $14.17(0.06)$ & $\ldots$ & $\ldots$ & PAIRITEL (2MASS) \\
\hline 56055.71 & 576.71 & $14.22(0.06)$ & $\ldots$ & $11.98(0.08)$ & PAIRITEL (2MASS) \\
\hline 56058.71 & 579.71 & $\ldots$ & $12.97(0.07)$ & $\ldots$ & PAIRITEL (2MASS) \\
\hline 56060.68 & 581.68 & $14.26(0.06)$ & $\ldots$ & $\ldots$ & PAIRITEL (2MASS) \\
\hline 56061.67 & 582.67 & $14.30(0.06)$ & $12.99(0.07)$ & $\ldots$ & PAIRITEL (2MASS) \\
\hline 56066.65 & 587.65 & $14.23(0.06)$ & $\ldots$ & $\ldots$ & PAIRITEL (2MASS) \\
\hline 56217.02 & 738.02 & $15.03(0.06)$ & $\ldots$ & $\ldots$ & PAIRITEL (2MASS) \\
\hline 56226.02 & 747.02 & $14.99(0.06)$ & $13.50(0.07)$ & $12.37(0.08)$ & PAIRITEL (2MASS) \\
\hline 56228.02 & 749.02 & $15.09(0.06)$ & $\ldots$ & $\ldots$ & PAIRITEL (2MASS) \\
\hline 56229.00 & 750.00 & $\ldots$ & $13.58(0.07)$ & $\ldots$ & PAIRITEL (2MASS) \\
\hline 56231.97 & 752.97 & $15.12(0.06)$ & $\ldots$ & $12.29(0.08)$ & PAIRITEL (2MASS) \\
\hline 56344.39 & 865.39 & $15.80(0.06)$ & $13.77(0.07)$ & $12.60(0.08)$ & NOT (NOTCAM) \\
\hline 56374.44 & 895.44 & $15.90(0.06)$ & $14.02(0.07)$ & $12.73(0.08)$ & NOT (NOTCAM) \\
\hline 56621.69 & 1142.7 & $17.28(0.25)$ & $14.40(0.11)$ & $12.99(0.12)$ & NOT (NOTCAM) \\
\hline
\end{tabular}


Table 3

Log of HST Observations for SN 2010j1

\begin{tabular}{|c|c|c|c|c|c|c|c|}
\hline Date & $\begin{array}{c}\text { J.D. } \\
2,450,000+\end{array}$ & $\begin{array}{l}\text { Epoch } \\
(\text { days })^{\mathrm{a}}\end{array}$ & Instrument & Grism/Grating & $\begin{array}{l}\text { Wavelength } \\
\text { Range (§) }\end{array}$ & $\begin{array}{c}\text { Spectral } \\
\text { Resolution }\end{array}$ & $\begin{array}{c}\text { Exposure } \\
\mathrm{s}\end{array}$ \\
\hline 2010 Nov 11 & 5512.37 & 33.9 & STIS & G230LB & $1685-3065$ & $615-1135$ & 3550 \\
\hline \multirow[t]{3}{*}{2010 Nov 22} & 5522.69 & 44.2 & COS & G130M & $1150-1450$ & $16,000-21,000$ & 1000 \\
\hline & 5522.70 & & $\mathrm{COS}$ & G160M & $1405-1775$ & $16,000-21,000$ & 1000 \\
\hline & 5522.56 & & STIS & G230LB & $1685-3065$ & $615-1135$ & 3575 \\
\hline \multirow[t]{4}{*}{2011 Jan 23} & 5585.33 & 106.8 & COS & G130M & $1150-1450$ & $16,000-21,000$ & 2380 \\
\hline & 5585.39 & & $\mathrm{COS}$ & G160M & $1405-1775$ & $16,000-21,000$ & 2950 \\
\hline & 5585.13 & & STIS & G230LB & $1685-3065$ & 615-1135 & 2300 \\
\hline & 5585.27 & & STIS & G430L & $2900-5700$ & $530-1040$ & 1200 \\
\hline \multirow[t]{2}{*}{2012 May 3} & 6051.45 & 573.0 & STIS & G230L & $1568-3184$ & $615-1135$ & 4400 \\
\hline & 6051.39 & & STIS & G430L & $2900-5700$ & $530-1040$ & 600 \\
\hline 2012 Jun 20 & 6098.75 & 620.6 & COS & G130M & $1150-1450$ & $16,000-21,000$ & 7400 \\
\hline
\end{tabular}

Note. ${ }^{\text {a }}$ Relative to first detection date, 2010 October 9, JD 2,455,479.

A large number of optical spectra (3400-7300 $)$ were also obtained at the FLWO $1.5 \mathrm{~m}$ Tillinghast telescope using the FAST spectrograph (Fabricant et al. 1998) from day 28 to day 767. FLWO/FAST data are reduced using a combination of standard IRAF and custom IDL procedures (Matheson et al. 2005). High-resolution spectra were also obtained with TRES (Tillinghast Reflector Echelle Spectrograph), which is a fiberfed echelle spectrograph on the $1.5 \mathrm{~m}$ Tillinghast telescope at FLWO.

Moderate-resolution optical spectra were obtained with the $2.4 \mathrm{~m}$ Hiltner telescope at MDM Observatory, on Kitt Peak, Arizona, on day 597. The Ohio State Multi-Object Spectrograph (OSMOS; Martini et al. 2011) was used with the VPH grism and 1".4 inner slit in combination with the MDM4K CCD detector. These spectra were reduced and calibrated by employing standard techniques in IRAF. Cosmic rays and obvious cosmetic defects have been removed. Wavelengths were checked against night-sky emission lines, and flux calibrations were applied using observations of Stone (1977) and Massey \& Gronwall (1990) standard stars.

The $6.5 \mathrm{~m}$ MMT spectra were all taken by the Blue Channel spectrograph. All observations used the 1".0 slit oriented at the parallactic angle. The reduction procedure was the same as for the FLWO/FAST spectra.

One medium-resolution spectrum with X-shooter at the Very Large Telescope (VLT) at ESO on day 461 (2012 January 14) was obtained, including both the optical and NIR ranges. The slit used was 1".0 in the UV and $0^{\prime \prime} \cdot 9$ in the optical and NIR. The resolutions were 4350, 7450, and 5300 in the UV, optical, and NIR, respectively, corresponding to 69,40 , and $56 \mathrm{~km} \mathrm{~s}^{-1}$, respectively. The $\mathrm{X}$-shooter spectrum was prereduced using version 1.1.0 of the dedicated ESO pipeline (Goldoni et al. 2006), with calibration frames (biases, darks, flat fields, and arc lamps) taken during daytime. Spectrum extraction and flux calibration were done using standard IRAF tasks. For the latter we used a spectrophotometric standard taken from the ESO list (http://www.eso.org/sci/facilities/paranal/instruments/xshooter/ tools/specphot_list.html) and observed during the same night. Telluric bands were removed using a telluric standard spectrum taken at the same air mass as the SN.

Absolute fluxes of all of our spectra were determined from $r^{\prime}$ band photometry. With the strong $\mathrm{H} \alpha$ line from the supernova, this is the band least affected by the galaxy background. For the last epoch MMT spectrum at 1128 days, we do not have any simultaneous optical photometry, and we estimate that the absolute flux is only accurate to $\sim 25 \%$.

\section{RESULTS}

\subsection{Reddening}

From Schlegel et al. (1998), Smith et al. (2011a) estimate a Milky Way reddening corresponding to $E_{(\mathrm{B}-\mathrm{V})}=0.027 \mathrm{mag}$. Based on the weak Na I lines, Smith et al. (2011a) assume negligible reddening from the host galaxy. From the damping wings of the $\operatorname{Ly} \alpha$ absorption in our COS spectra, we can make an independent estimate of this for both the host and for the Milky Way.

We discuss the COS spectrum in detail later. Here we use the damping wings of the Ly $\alpha$ absorption to derive a value for the column density, $N_{H}$ (e.g., Draine 2011, Chapter 9.4). The Milky Way absorption is affected by the Si III $\lambda 1206.5$ absorption on the red side, so we use the blue side for the fit. For the host galaxy we instead use the red side to avoid the slight overlap with the Milky Way absorption. From fits of these, shown in Figure 1, we derive a value of $N_{\mathrm{H}}=(1.05 \pm 0.3) \times 10^{20} \mathrm{~cm}^{-2}$ for $b=10 \mathrm{~km} \mathrm{~s}^{-1}$ for the host galaxy, and the corresponding value is $\mathrm{N}(\mathrm{HI})_{\mathrm{MW}}=1.75( \pm 0.25) \times 10^{20} \mathrm{~cm}^{-2}$ from the Milky Way. There is some uncertainty on the Milky Way column density because the Si III $1206.5 \AA$ absorption line from the host galaxy falls on the red edge of the Milky Way Ly $\alpha$ profile.

Using the $N_{\mathrm{HI}}$ versus $E_{(\mathrm{B}-\mathrm{V})}$ relation in Bohlin et al. (1978), and assuming the same $N\left(\mathrm{H}_{2}\right) / N(\mathrm{H} \mathrm{I})$ ratio for the host galaxy of $2010 \mathrm{jl}$ as in the Milky Way, we find $E_{(\mathrm{B}-\mathrm{V})}=0.036 \mathrm{mag}$ for the Milky Way and $E_{(\mathrm{B}-\mathrm{V})}=0.022 \mathrm{mag}$ for the host galaxy. The value for the Milky Way agrees well with that derived from the far-IR (FIR) emission, but we also note that there is a nonnegligible absorption from the host galaxy. For the remainder of the paper, where needed, we will adopt a value of $E_{(\mathrm{B}-\mathrm{V})}=0.058 \mathrm{mag}$ for the total reddening.

\subsection{Light Curves and Total Energy Output}

Figure 2 shows the light curve from our photometry, complemented with early measurements from Stoll et al. (2011). The $B, V$, and $i^{\prime}$ bands all show nearly the same slow decline by 
Table 4

Journal of Spectroscopic Observations

\begin{tabular}{|c|c|c|c|c|c|c|c|}
\hline UT Date & J.D.M & $\begin{array}{c}\text { Epoch }^{\mathrm{a}} \\
\text { (days) }\end{array}$ & $\begin{array}{c}\text { Range } \\
(\AA)\end{array}$ & $\begin{array}{c}\text { FWHM Res. } \\
(\AA)\end{array}$ & $\begin{array}{c}\text { Exposure } \\
\text { (s) }\end{array}$ & Airmass & Instrument \\
\hline 2010 Nov 7 & 5507.53 & 28.5 & $3475-7415$ & 6.2 & 240 & 1.13 & FAST/300GPM \\
\hline 2010 Nov 9 & 5509.71 & 30.7 & $6350-6850$ & 1.2 & $600 \times 4$ & 1.30 & ALFOSC/gr17 \\
\hline 2010 Nov 10 & 5510.72 & 31.7 & $3500-5060$ & 1.9 & $400 \times 3$ & 1.25 & ALFOSC/gr16 \\
\hline 2010 Nov 14 & 5515.4 & 36.4 & $3177-8527$ & 6.5 & 120 & 1.72 & $\mathrm{MMT} / 300 \mathrm{GPM}$ \\
\hline 2010 Nov 15 & 5516.4 & 37.4 & $3188-8534$ & 6.5 & 120 & 1.14 & MMT/300GPM \\
\hline 2010 Nov 16 & 5517.4 & 38.4 & $3186-4516$ & 1.45 & 1200 & 1.40 & MMT/1200GPM \\
\hline 2010 Nov 16 & 5517.5 & 38.5 & $4395-5731$ & 1.45 & 750 & 1.35 & MMT/1200GPM \\
\hline 2010 Nov 16 & 5517.5 & 38.5 & $5587-6931$ & 1.45 & 600 & 1.30 & MMT/1200GPM \\
\hline 2010 Nov 16 & 5517.5 & 38.5 & 3177-8399 & 6.5 & 120 & 1.15 & MMT/300GPM \\
\hline 2010 Nov 18 & 5518.68 & 39.7 & $6350-6850$ & 1.2 & $600 \times 2$ & 1.37 & ALFOSC/gr17 \\
\hline 2010 Nov 18 & 5518.71 & 39.7 & $3500-5060$ & 1.9 & $500 \times 3$ & 1.21 & ALFOSC/gr16 \\
\hline 2010 Nov 24 & 5524.76 & 45.8 & $6350-6850$ & 1.2 & $600 \times 2$ & 1.07 & ALFOSC/gr17 \\
\hline 2010 Nov 24 & 5524.72 & 45.7 & $3500-5060$ & 2.9 & $500 \times 3$ & 1.13 & ALFOSC/gr16 \\
\hline 2010 Nov 28 & 5529.4 & 50.4 & $5653-7538$ & 2.0 & 900 & 1.4 & $\mathrm{MMT} / 832 \mathrm{GPM}$ \\
\hline 2010 Dec 9 & 5540.40 & 61.4 & $5575-6908$ & 1.45 & 450 & 1.1 & MMT/1200GPM \\
\hline 2010 Dec 11 & 5541.50 & 62.5 & $3475-7415$ & 6.2 & 900 & 1.08 & FAST/300GPM \\
\hline 2010 Dec 12 & 5542.47 & 63.5 & $3475-7415$ & 6.2 & 1020 & 1.09 & FAST/300GPM \\
\hline 2010 Dec 16 & 5546.41 & 67.4 & $3850-9000$ & 0.56 & 3600 & 1.14 & TRES \\
\hline 2010 Dec 19 & 5549.47 & 70.5 & $3850-9000$ & 0.56 & 3000 & 1.08 & TRES \\
\hline 2010 Dec 28 & 5558.37 & 79.4 & $3850-9000$ & 0.56 & 3600 & 1.17 & TRES \\
\hline 2010 Dec 28 & 5558.63 & 79.6 & $6350-6850$ & 1.2 & $600 \times 3$ & 1.13 & ALFOSC/gr17 \\
\hline 2010 Dec 28 & 5558.65 & 79.7 & $3500-5060$ & 2.9 & $600 \times 3$ & 1.08 & ALFOSC/gr16 \\
\hline 2011 Jan 2 & 5563.36 & 84.4 & $3475-7415$ & 6.2 & 900 & 1.19 & FAST/300GPM \\
\hline 2011 Jan 5 & 5566.43 & 87.4 & $3475-7415$ & 6.2 & 1020 & 1.08 & FAST/300GPM \\
\hline 2011 Jan 8 & 5569.39 & 90.4 & $3475-7415$ & 6.2 & 900 & 1.09 & FAST/300GPM \\
\hline 2011 Jan 13 & 5574.45 & 95.5 & $3850-9000$ & 0.56 & 3600 & 1.15 & TRES \\
\hline 2011 Jan 28 & 5589.29 & 110.3 & $3475-7415$ & 6.2 & 300 & 1.20 & FAST/300GPM \\
\hline 2011 Jan 31 & 5592.55 & 113.6 & $6350-6850$ & 1.2 & $600 \times 4$ & 1.10 & ALFOSC/gr17 \\
\hline 2011 Jan 31 & 5592.59 & 113.6 & $3500-5060$ & 1.9 & $600 \times 2$ & 1.06 & ALFOSC/gr16 \\
\hline 2011 Feb 5 & 5597.41 & 118.4 & $3475-7415$ & 6.2 & 1200 & 1.18 & FAST/300GPM \\
\hline 2011 Mar 1 & 5621.5 & 142.5 & $6350-6850$ & 1.2 & $1200 \times 3$ & 1.18 & ALFOSC/gr17 \\
\hline 2011 Mar 3 & 5623.23 & 144.2 & $3475-7415$ & 6.2 & 1544 & 1.10 & FAST/300GPM \\
\hline 2011 Mar 9 & 5629.21 & 150.2 & $3475-7415$ & 6.2 & 900 & 1.11 & FAST/300GPM \\
\hline 2011 Mar 10 & 5630.56 & 151.6 & $3500-5060$ & 1.9 & $900 \times 1$ & 1.17 & ALFOSC/gr16 \\
\hline 2011 Mar 16 & 5636.23 & 157.2 & $3475-7415$ & 6.2 & 900 & 1.08 & FAST/300GPM \\
\hline 2011 Mar 29 & 5650.41 & 171.4 & $6350-6850$ & 1.2 & $900 \times 3$ & 1.07 & ALFOSC/gr17 \\
\hline 2011 Apr 3 & 5654.18 & 175.2 & $3475-7415$ & 6.2 & 819 & 1.08 & FAST/300GPM \\
\hline 2011 Apr 8 & 5660.41 & 181.4 & $3500-5060$ & 1.9 & $900 \times 3$ & 1.06 & ALFOSC/gr16 \\
\hline 2011 May 19 & 5701.40 & 222.4 & $6350-6850$ & 1.2 & $900 \times 3$ & 1.31 & ALFOSC/gr17 \\
\hline 2011 May 21 & 5703.39 & 224.4 & $3500-5060$ & 1.9 & $900 \times 2$ & 1.29 & ALFOSC/gr16 \\
\hline 2011 Oct 28 & 5862.52 & 383.5 & $3475-7415$ & 6.2 & 1010 & 1.28 & FAST/300GPM \\
\hline 2011 Oct 30 & 5864.48 & 385.5 & $3475-7415$ & 6.2 & 1200 & 1.44 & FAST/300GPM \\
\hline 2011 Nov 3 & 5868.49 & 389.5 & $3475-7415$ & 6.2 & 1800 & 1.33 & FAST/300GPM \\
\hline 2011 Nov 28 & 5894.5 & 415.5 & $5653-7538$ & $0.71 / \mathrm{px}$ & 900 & 1.2 & $\mathrm{MMT} / 832 \mathrm{GPM}$ \\
\hline 2011 Dec 24 & 5919.51 & 440.5 & $3475-7415$ & 6.2 & 1800 & 1.15 & FAST/300GPM \\
\hline 2011 Dec 27 & 5922.36 & 443.4 & $3475-7415$ & 6.2 & 1800 & 1.26 & FAST/300GPM \\
\hline 2011 Dec 31 & 5926.31 & 447.3 & $3475-7415$ & 6.2 & 1800 & 1.47 & FAST/300GPM \\
\hline 2012 Jan 14 & 5940.23 & 461.2 & 3000-10200 & 0.1 & 5376 & $1.22-1.41$ & X-SHOOTER \\
\hline 2012 Jan 14 & 5940.23 & 461.2 & $10200-24800$ & 0.3 & 5928 & $1.22-1.41$ & X-SHOOTER \\
\hline 2012 Jan 18 & 5944.32 & 465.3 & $3475-7415$ & 6.2 & 1800 & 1.16 & FAST/300GPM \\
\hline 2012 Mar 24 & 6010.15 & 531.2 & $3475-7415$ & 6.2 & 1800 & 1.14 & FAST/300GPM \\
\hline 2012 May 12 & 6059.15 & 580.2 & $3475-7415$ & 6.2 & 1800 & 1.22 & FAST/300GPM \\
\hline 2012 May 15 & 6062.18 & 583.2 & $3475-7415$ & 6.2 & 1800 & 1.43 & FAST/300GPM \\
\hline 2012 May 29 & 6076.18 & 597.2 & $4000-6900$ & 3.5 & $900 \times 3$ & 1.9 & MDM/OSMOS \\
\hline 2012 Jun 15 & 6093.16 & 614.2 & $3475-7415$ & 6.2 & 1200 & 2.40 & FAST/300GPM \\
\hline 2012 Oct 20 & 6221.00 & 742.0 & $3475-7415$ & 6.2 & 1800 & 1.38 & FAST/300GPM \\
\hline 2012 Nov 12 & 6244.00 & 765.0 & $3475-7415$ & 6.2 & 1200 & 1.10 & FAST/300GPM \\
\hline 2012 Nov 14 & 6246.00 & 767.0 & $3475-7415$ & 6.2 & 1800 & 1.20 & FAST/300GPM \\
\hline 2012 Dec 21 & 6282.8 & 803.8 & $5587-6931$ & 1.45 & 1200 & 1.1 & $\mathrm{MMT} / 1200 \mathrm{GPM}$ \\
\hline 2013 Feb 2 & 6325.8 & 846.8 & $3177-8527$ & 6.5 & 1800 & 1.2 & MMT/300GPM \\
\hline 2013 Feb 3 & 6326.8 & 847.8 & $5587-6931$ & 1.45 & 1800 & 1.3 & $\mathrm{MMT} / 1200 \mathrm{GPM}$ \\
\hline 2013 Nov 10 & 6606.7 & 1127.7 & $6400-6900$ & 1.45 & 1800 & 1.3 & $\mathrm{MMT} / 1200 \mathrm{GPM}$ \\
\hline
\end{tabular}

Note. ${ }^{\text {a }}$ Relative to first detection date, JD 2,455,479.0. 


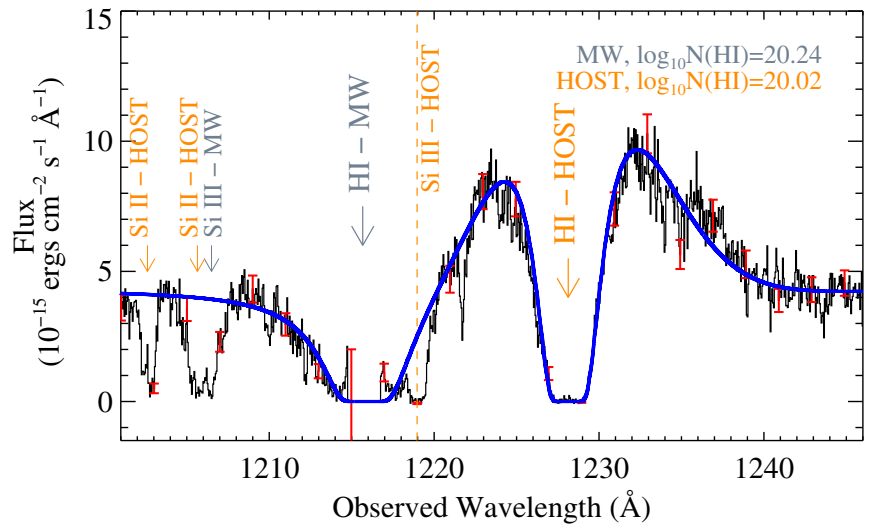

Figure 1. Fit to the Ly $\alpha$ absorptions on 2011 January 23 from the Milky Way and from the host galaxy. The blue line shows the fit, the black line shows the observations, and the red error bars the one-sigma error.

(A color version of this figure is available in the online journal.)

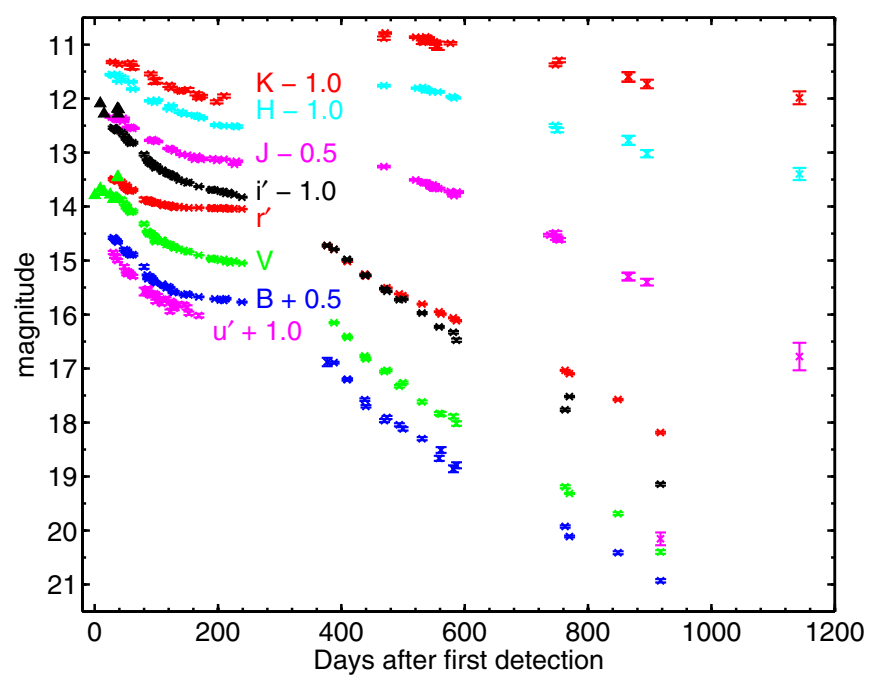

Figure 2. Light curves of SN 2010j1 in different bands. Triangles are early, prediscovery $I$ - and $V$-band observations from Stoll et al. (2011).

(A color version of this figure is available in the online journal.)

$\sim 0.8 \mathrm{mag} / 100$ days during the first $\sim 175$ days. After this the light curves become almost constant up to $\sim 240$ days. However, the $r^{\prime}$ band already shows signs of flattening at age 100 days. We discuss this band, dominated by $\mathrm{H} \alpha$, in more detail in Section 3.4. Being most sensitive to the decreasing temperature, the $u^{\prime}$ band shows the steepest decline.

After the first gap produced by conjunction with the Sun, all optical bands show a considerably faster decline at $\gtrsim 300$ days. In the period 400-850 days, the decline in the optical bands is roughly linear. The decline rate is $8.0 \times 10^{-3} \mathrm{mag} \mathrm{day}^{-1}$ in $i^{\prime}$, $4.9 \times 10^{-3} \mathrm{mag} \mathrm{day}^{-1}$ in $r^{\prime}, 8.0 \times 10^{-3} \mathrm{mag} \mathrm{day}^{-1}$ in $\mathrm{V}$, and $7.1 \times 10^{-3}$ mag day $^{-1}$ in $\mathrm{B}$. We attribute the slow $r$-band decline to emission in $\mathrm{H} \alpha$ : as seen in the spectra, $\mathrm{H} \alpha$ is stronger with respect to the continuum as time goes by (Section 3.3).

Compared to the $R$-band photometry of Ofek et al. (2014), we agree within less than 0.1 mag over the whole period. Up to day 200 our optical photometry also agrees within $\sim 0.1-0.2$ mag in the $r^{\prime}, B$, and $V$ bands with the light curves by Zhang et al. (2012), in spite of the difference in filter profiles between our $r^{\prime}$ band and their $R$ band. This is probably because the $r^{\prime}$ and $R$ bands are both dominated by $\mathrm{H} \alpha$. Our $i^{\prime}$ magnitudes are $\sim 0.5$ mag fainter than the $I$ magnitudes by Zhang et al. and

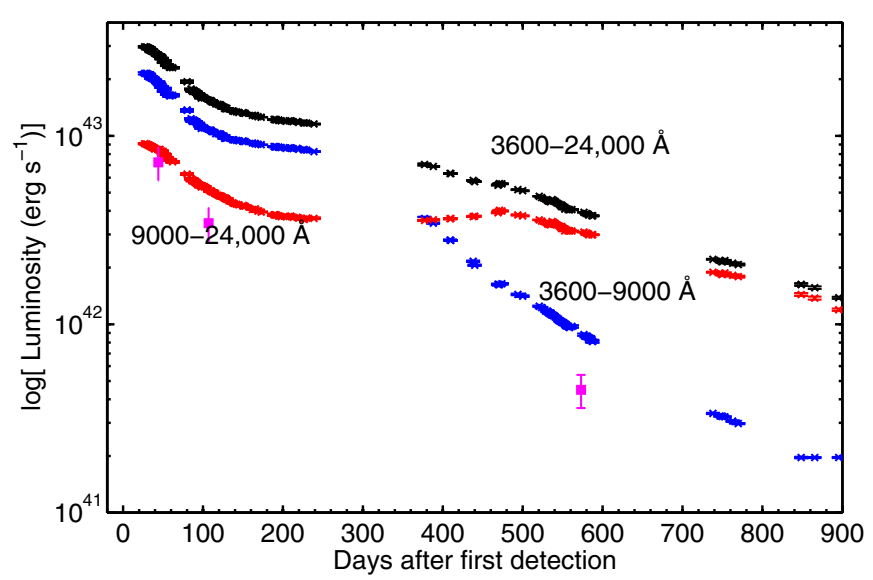

Figure 3. Pseudobolometric light curves of SN 2010j1. The contributions from the 3600-9000 $\AA$ and 9000-24,000 Å ranges are shown separately. Also shown as magenta squares are the UV luminosities at the time of our HST observations. The day 44 and day 107 luminosities include the 1100-3600 ̊ range, but day 573 only includes the STIS 1700-3600 Å range.

(A color version of this figure is available in the online journal.)

$\sim 0.3$ mag fainter in $u^{\prime}$ compared to their $U$ band. Most likely this difference can be explained by the different filter responses between the SDSS $i^{\prime}$ and $u^{\prime}$ bands and the standard $I$ and $U$ bands.

At epochs later than 350 days, our magnitudes are 0.5-1.5 mag fainter in all bands, a difference that increases with time. The origin of this is not clear, but we note that Zhang et al. do not give any errors in their table of the photometry for these epochs. Also, our photometry showed a similar flattening of the light curve until we subtracted the background from the host galaxy.

The NIR light curves show a similar decline to the optical up to $\sim 200$ days. After this, the light curves flatten considerably in $J$ and $H$, and the $K$ band shows an increase compared to the flux at earlier times. The difference between the NIR and the optical behavior is real and has important consequences for our physical picture for SN 2010jl. As we discuss in Section 4.2, we interpret this emission as coming from dust in the CSM of the progenitor.

The pseudobolometric light curve for the flux in the wavelength range 3500-25,000 was calculated using the method described in Ergon et al. (2014). In the optical region where we have a well-sampled spectral sequence, we made use of both photometry and spectroscopy to accurately calculate the bolometric luminosity, whereas in the NIR region we used photometry to interpolate the shape of the SED. The SED has then been dereddened using $E_{(\mathrm{B}-\mathrm{V})}=0.036 \mathrm{mag}$ for the Milky Way and $E_{(\mathrm{B}-\mathrm{V})}=0.022$ mag for the host galaxy, as derived in Section 3.1. For the Milky Way reddening, we use the extinction law by Cardelli et al. (1989), and being a star-forming galaxy with Wolf-Rayet (WR) features (Shirazi \& Brinchmann 2012), we use the starburst extinction from Calzetti et al. (1994) for the host galaxy.

As is clear from Figure 2, the contribution from the NIR bands becomes increasingly important. The luminosity in the NIR region (9000-24,000 $\AA$ ) is shown in Figure 3 as red points, whereas the total (3500-25,000 $\mathrm{A}$ ) luminosity is shown as black points. While the optical bolometric contribution starts to drop at $\sim 350$ days, the NIR part has a long plateau from 200 to $\sim 450$ days. At age 100 days, the IR represents $33 \%$ of the flux, equaling the optical output at day 400; by age 
500 days, it is $73 \%$, and by day $770,85 \%$. This era is dominated by a dust contribution (Section 4.2) and reflects both the instantaneous energy output and a contribution from a light echo. In Section 4.2, we show that the SN itself dominates the NIR at early epochs, but later the NIR comes from an echo.

The pseudobolometric light curve in Figure 3 only includes the optical and NIR bands and ignores both the UV and mid-IR. Especially at the early phases, the comparatively hot spectrum has a large UV contribution. We will discuss the spectra in more detail later; here we only estimate this contribution from an integration of the flux from COS and STIS for days 44, 107, and 573. We do not include the COS spectrum for day 621 because this is severely contaminated by the host galaxy.

On day 44 we find a luminosity in the $1100-3600 \AA$ range equal to $7.2 \times 10^{42} \mathrm{erg} \mathrm{s}^{-1}$, and for day 107 it is $3.4 \times 10^{42} \mathrm{erg} \mathrm{s}^{-1}$. For day 573 we find a luminosity of $4.5 \times 10^{41} \mathrm{erg} \mathrm{s}^{-1}$ in the STIS $1700-3600 \AA$ range. We estimate the uncertainty in these fluxes as $\sim 25 \%$. There is therefore a substantial UV contribution to the bolometric luminosity, especially at late epochs, up to $\sim 50 \%$ of the optical luminosity at the last epoch. There may also be a substantial EUV and X-ray luminosity that is not included in our observations.

Up to day $\sim 200$, our total bolometric luminosity agrees with that of Zhang et al. (2012). Zhang et al. have, however, no NIR (or UV) observations. These account for an increasing fraction of the luminosity as the event ages, so their luminosities are increasingly incomplete. In addition, the background contribution from the host is very important after day $\sim 350$, as was discussed above. At early epochs there is some discrepancy between our bolometric luminosities and those of Zhang et al. It is not clear what produces this difference: it may be due to Zhang et al. using photometry alone, while we use a combination of photometry and spectroscopy to combine the bands.

Assuming that the luminosity is the same as at our first determinations at day 27 (see below), we find a total integrated energy from day 0 to day 920 in the $3600-9000 \AA$ range of $3.3 \times 10^{50} \mathrm{erg}$ and in the $9000-24,000 \AA$ range of $2.6 \times 10^{50} \mathrm{erg}$. The total in the $3600-24,000 \AA$ range is therefore $5.8 \times 10^{50} \mathrm{erg}$. The correct way to use the IR measurements depends on the interpretation (Section 4.2) and on the relative contributions between the photosphere emission and dust: NIR light from an echo reflects the optical-UV output, while NIR light from shock-heated dust should be added to the energy budget.

To disentangle the dust and direct photospheric luminosity from the SN, we have made fits to the SED from the photometry for most epochs. We have here included the Spitzer 3.6 and $4.5 \mu \mathrm{m}$ fluxes from Andrews et al. (2011) at day 87 (JD 2,455,565) and from Fox et al. (2013) for day 254 (JD 2,455,733), as well as unpublished Spitzer observations from the Spitzer archive from days 465, 621, and 844. For these epochs we find fluxes of 8.55, 8.63, and $7.73 \mathrm{mJy}$ at $3.6 \mu \mathrm{m}$ and $8.30,8.66$, and $8.24 \mathrm{mJy}$ at $4.5 \mu \mathrm{m}$.

In Figure 4 we show the SEDs for these dates, as well as for one epoch (750 days) where we only have optical and NIR data. These SEDs are modeled with two blackbodies with different temperatures, and we minimize the chi square with the photospheric effective temperature and radius $T_{\text {eff }}$ and $R_{\text {phot }}$ and the corresponding parameters for the dust shell, $T_{\text {dust }}$ and $R_{\text {dust }}$, as free parameters. We further discuss the motivation for this choice of spectrum in Section 4.2. In these fits we exclude the $r$ band because it is dominated by the $\mathrm{H} \alpha$ line. For the Spitzer photometry at days 254,621 , and 844 , where no other

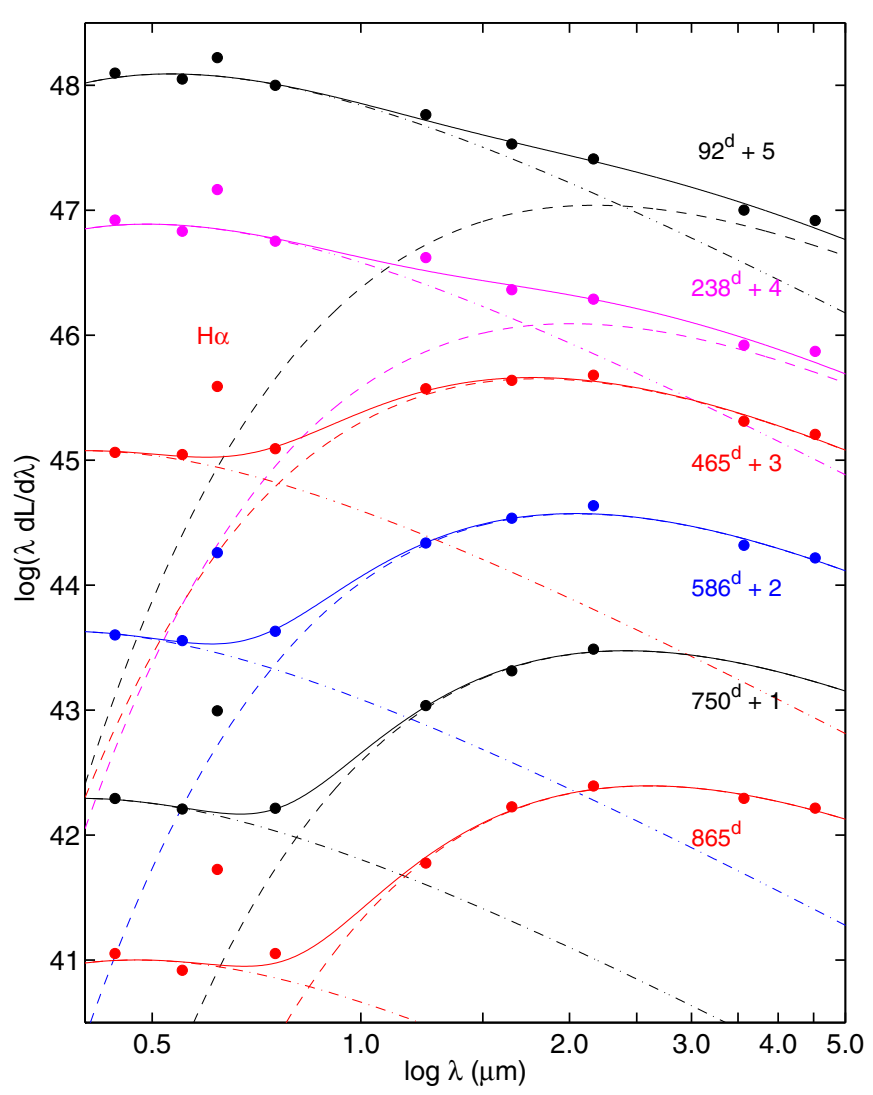

Figure 4. Spectral energy distributions at different epochs, together with blackbody fits from a dust component and a photospheric component. Because of the dominance of the $\mathrm{H} \alpha$ line in the $r$ band, this band is not included in the fits. For clarity, each spectrum has been shifted by one decade in luminosity relative to the previous.

(A color version of this figure is available in the online journal.)

Table 5

Parameters for the SED Fits Assuming Two Blackbody Components

\begin{tabular}{lcccc}
\hline \hline $\begin{array}{l}\text { Epoch } \\
\text { (days) }\end{array}$ & $\begin{array}{l}T_{\text {eff }} \\
(\mathrm{K})\end{array}$ & $\begin{array}{c}R_{\text {phot }} \\
\left(10^{15} \mathrm{~cm}\right)\end{array}$ & $\begin{array}{c}T_{\text {dust }} \\
(\mathrm{K})\end{array}$ & $\begin{array}{c}R_{\text {dust }} \\
\left(10^{16} \mathrm{~cm}\right)\end{array}$ \\
\hline 92 & 6900 & 3.20 & 1685 & 1.60 \\
238 & 7450 & 2.18 & 1830 & 1.44 \\
465 & 9200 & 0.56 & 2040 & 2.21 \\
586 & 9900 & 0.29 & 1790 & 2.61 \\
750 & 9300 & 0.22 & 1520 & 3.24 \\
880 & 7750 & 0.23 & 1410 & 3.43 \\
\hline
\end{tabular}

photometry exists, we interpolate the Spitzer fluxes and the NIR and optical photometry to days 238,586 , and 865 , respectively. Given the slow evolution of the Spitzer fluxes at these epochs, this should only introduce a minor error.

From these models we find that up to day $\sim 400$ the photospheric contribution also dominates the NIR, and at later stages the dust component takes over. The best-fit parameters for these models are given in Table 5 for the different dates, and in Figure 5 we show the blackbody temperature, radius, and total luminosity of the dust and photospheric components from these fits, together with the standard deviations. The large errors in $T_{\text {dust }}$ and $R_{\text {dust }}$ at the first two epochs are a result of the dominance of the photospheric component also for the NIR, and the errors in $T_{\text {eff }}$ are mainly a result of the relatively few bands (mainly $B, V$, and $I$ ) where there is a large contribution from this component. 


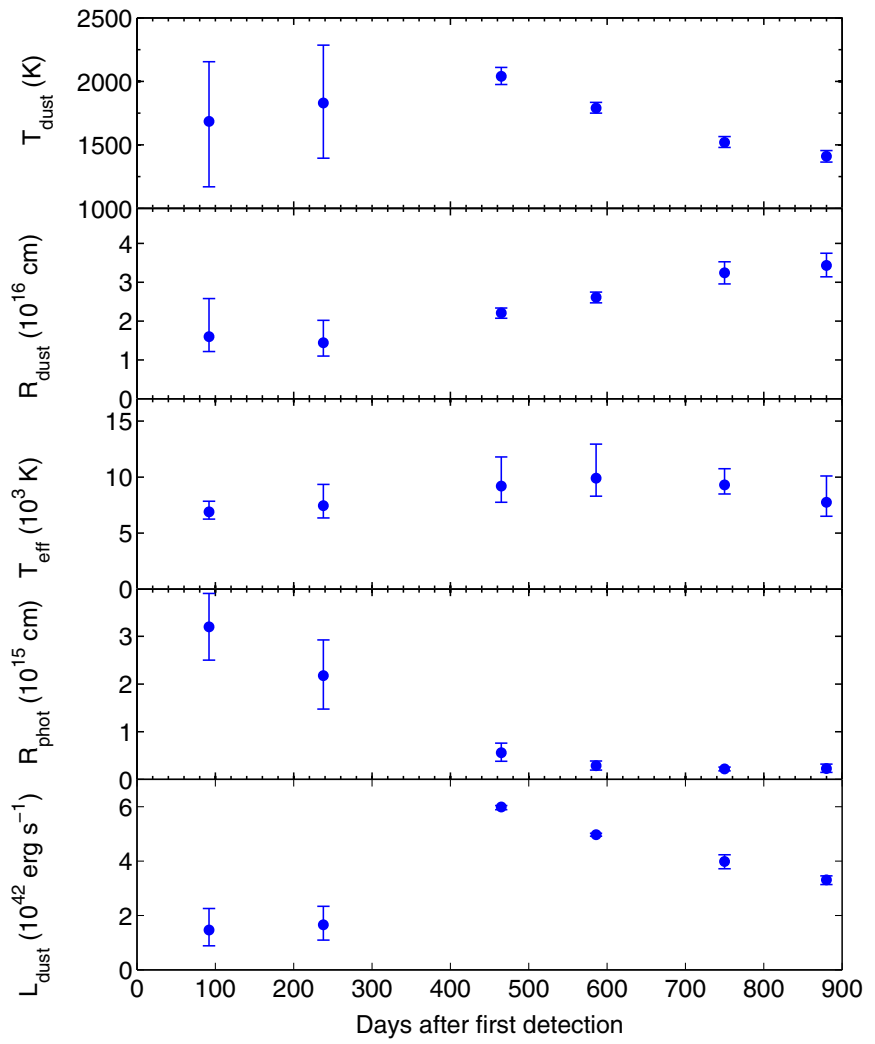

Figure 5. Blackbody temperature, radius, and luminosity for the dust component and radius and effective temperature for the $\mathrm{SN}$ component for the epochs in Figure 4.

(A color version of this figure is available in the online journal.)

At epochs earlier than $\sim 400$ days, the dust temperature is constant within errors at $\sim 1850 \pm 200 \mathrm{~K}$ and then slowly decays to $\sim 1400 \mathrm{~K}$ at 850 days. The blackbody radius is $\sim(1-2) \times 10^{16} \mathrm{~cm}$ for the first $\sim 300$ days and then slowly increases to $\sim 3 \times 10^{16} \mathrm{~cm}$ at the last observation. The dust luminosities we obtain for the first epochs are lower than the NIR luminosities in Figure 3. The reason for this, as can be seen in Figure 4, is that the photospheric contribution dominates the $J, H$, and $K$ bands for these epochs. At epochs later than the day 465 observation, the opposite is true, which is a result of including the total dust emission from the blackbody fit and not only the NIR bands.

Already at 90 days, Andrews et al. (2011) found from NIR and Spitzer observations an IR excess due to warm dust, but with a lower temperature of $\sim 750 \mathrm{~K}$ than we find. Andrews et al., however, only include the Spitzer fluxes to the dust component, while we also include the $J, H$, and $K$ bands in this component, which explains our higher dust temperatures. We note that Andrews et al. (2011) underestimate the $K$-band flux in their SED fit.

Using the SED fitting, we can improve on the bolometric light curve by separating the $\mathrm{SN}$ and dust contributions of the IR flux to the bolometric luminosity and add this to the $B$ Vri contribution in Figure 3. Based on the UV flux at the epochs with $H S T$ observations, we multiply this by a factor of 1.25 (Section 3.2). In this way we arrive at the bolometric light curve from the SN ejecta alone in Figure 6, now shown in a log-log plot. From this we see that the bolometric light curve from the ejecta can be accurately characterized by a power-law decay from $\sim 20$ to 320 days, given by $L(t) \sim$

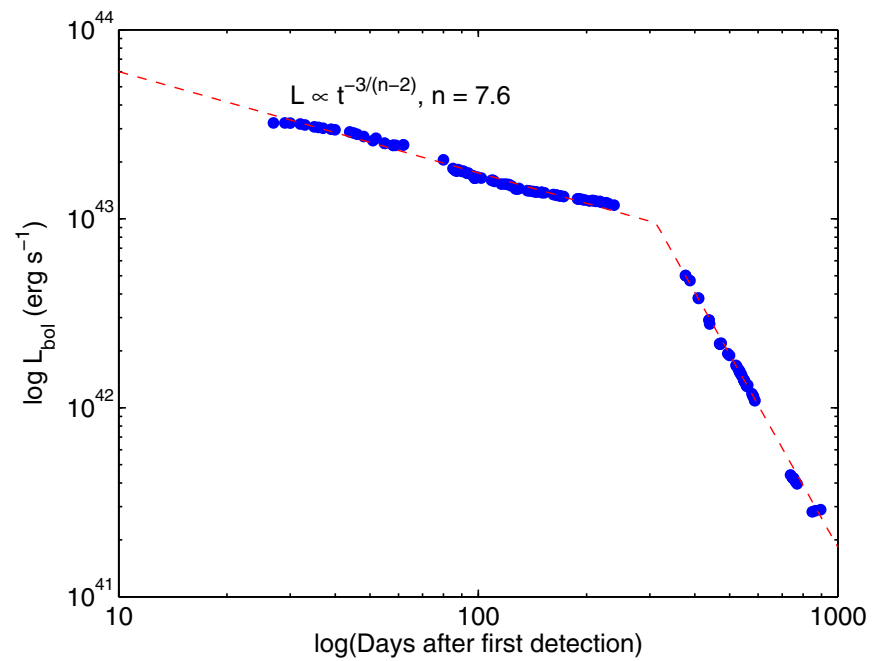

Figure 6. Bolometric light curve for the SN ejecta, excluding the dust echo. The dashed lines show power-law fits to the early and late light curves used to construct the density distribution of the explosion. Note the pronounced break in the light curve at $\sim 320$ days. The dashed lines give power-law fits to the luminosity before and after the break (see Section 4.5 for a discussion).

(A color version of this figure is available in the online journal.)

$1.75 \times 10^{43}(t / 100 \text { days })^{-0.536} \mathrm{erg} \mathrm{s}^{-1}$ and a final steep decay $L(t)=8.71 \times 10^{42}(t / 320 \text { days })^{-3.39} \mathrm{erg} \mathrm{s}^{-1}$ after day 320 .

Ofek et al. (2014) estimate the bolometric light curve by assuming a constant bolometric correction of -0.27 mag to the $R$-band photometry. With this assumption, they find a flatter light curve with $L(t) \propto t^{-0.36}$ for the same explosion date as we use here. The reason for this difference is that the $R$-band decays slower than most of the other bands, as can be seen from Figure 2. The bolometric light curve will therefore be steeper than the $R$-band light curve.

The slope depends on the assumed shock breakout date. Ofek et al. (2014) discuss this based on the light curve and find a likely range of 15-25 days before I-band maximum, corresponding to JD $2,455,4692,455,479$. Using $2,455,469$ instead of our $2,455,479$ would change the best-fit luminosity decline to $L(t) \sim 1.9 \times 10^{43}(t / 100 \text { days })^{-0.61} \mathrm{erg} \mathrm{s}^{-1}$.

To estimate the total energy output from the $\mathrm{SN}$, we assume that the bolometric luminosity before our first epoch at 26 days was constant at the level at 26 days, which is supported by the early observations by Stoll et al. (2011), shown in Figure 2. The total energy from the SN (excluding the echo) is then $6.5 \times 10^{50} \mathrm{erg}$. In addition, there is a contribution from the EUV as well as X-rays and mid-IR (Section 4.7). Even ignoring these, we note that the total radiated energy is a large fraction of the energy of a "normal" core-collapse SN (see Section 4.7).

\subsection{Spectroscopic Evolution}

Figures 7 and 8 show the SN 2010jl spectral sequence for days 29-847 obtained with FAST at FLWO and with grism 16 at NOT, respectively. The former have the advantage of showing the full spectral interval between 3500 and $7200 \AA$, and the NOT spectra below $5100 \AA$ have a higher dispersion, showing the narrow line profiles better.

From our first optical spectra at 29 days to the last at 848 days, we see surprisingly little change in the lines present (Figure 7). The main difference is that the continuum is getting substantially redder with time. This is also apparent from the steep light 


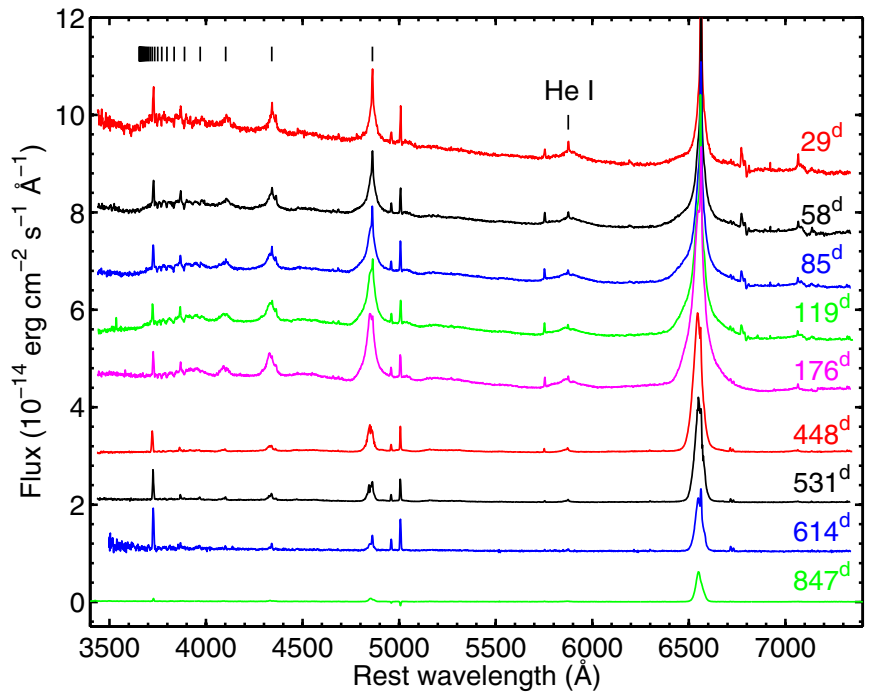

Figure 7. Spectral sequence in the optical from observations with FAST and MMT. Each spectrum has been shifted upward by $10^{-14} \mathrm{erg} \mathrm{s}^{-1} \mathrm{~cm}^{-2} \AA^{-1}$ relative to the one below. The wavelengths of the Balmer lines are shown, as well as the broad $\mathrm{He}$ I $\lambda 5876$ line.

(A color version of this figure is available in the online journal.)

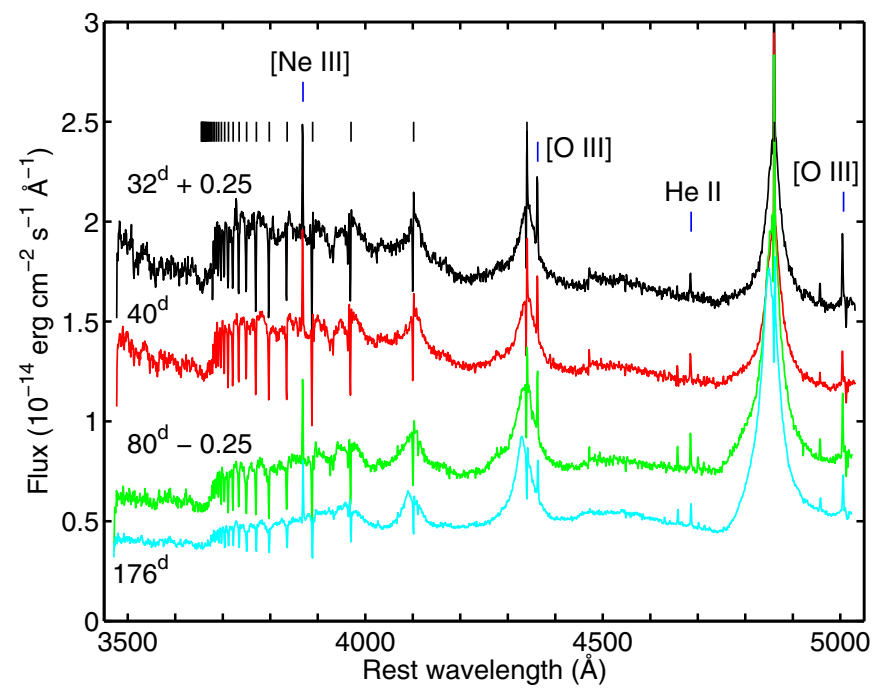

Figure 8. Grism 16 spectral series from NOT showing the Balmer series blueward of $\mathrm{H} \alpha$ as well as the Balmer decrement. Note the narrow lines due to [Ne III] $\lambda 3868.8,[\mathrm{O}$ III] $\lambda \lambda 4959,5007$, and He II $\lambda 4686$, and the unusually strong [O III] $\lambda 4363$ line. The day 32 and day 80 spectra have been shifted upward and downward, respectively, by $2.5 \times 10^{-15} \mathrm{erg} \mathrm{s}^{-1} \mathrm{~cm}^{-2} \AA^{-1}$.

(A color version of this figure is available in the online journal.)

curve of the $u^{\prime}$ band in Figure 2. At the same time, the Balmer discontinuity at $3640 \AA$ becomes weaker.

The most conspicuous features of the spectra are the strong, symmetric Balmer emission lines, from $\mathrm{H} \alpha$ up to $\mathrm{H} \delta$. To the blue of $\mathrm{H} \delta$, the broad emission lines blend into a continuum. In addition to these blended broad emissions in Figure 8, each Balmer line up to at least H16 shows a narrow blueshifted P-Cygni absorption. In addition to the Balmer lines and several He I lines, some of which show prominent P-Cygni profiles (Section 3.4.2), we also see narrow emission lines from [Ne III] $\lambda 3868$, [O III] $\lambda \lambda 4363,4959,5007$, several [Fe III] lines, as well as He II $\lambda 4686$ (for more details see Section 3.4.2). Our high-resolution $\mathrm{X}$-shooter spectrum reveals an additional large number of weaker narrow lines (Section 3.4.3).

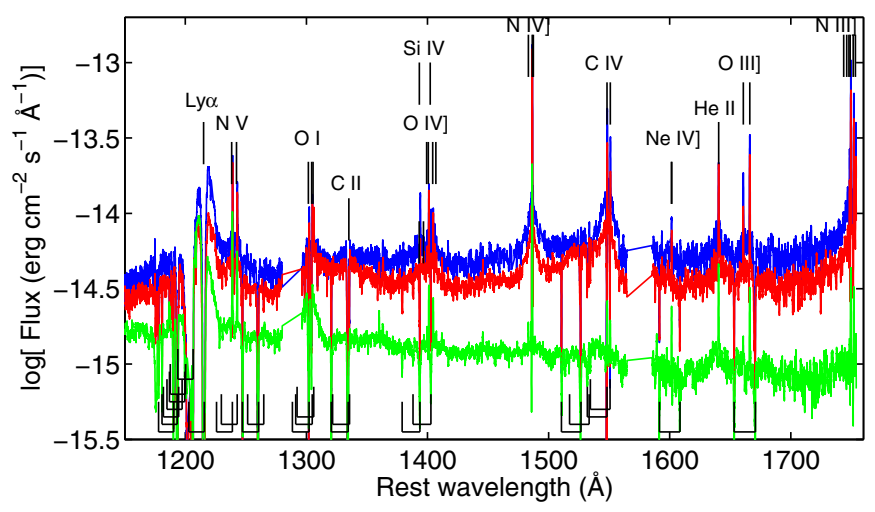

Figure 9. COS spectra from day 44 (blue), day 107 (red), and day 620 (green) with the most important lines marked. We also show the positions of the strongest absorptions from the Milky Way and host galaxy in the lower part of the figure. The continuum in the day 620 spectrum is dominated by the continuum emission from the host-galaxy background.

(A color version of this figure is available in the online journal.)

Figure 9 shows the far-UV spectrum with COS from days 44, 107, and 621, and Figure 10 shows the STIS spectra from days 34, 44, 107, and 573. Again, we see a number of prominent, broad lines with strong, narrow emission components but also in many cases P-Cygni absorptions. The main difference from the optical is that in the UV we find mainly lines of highly ionized elements, like C III-IV, N III-V, O III-IV, and Si III-IV, although there are also neutral and low-ionization lines of $\mathrm{C}$ II, O I, and $\mathrm{Mg}$ II. A comparison of the STIS spectra reveals little evolution of the spectrum between the first two epochs. The COS spectra show a similar slow evolution. At the time of the third epoch, the continuum has faded by a factor of $\sim 2$, while the narrow lines have decreased by a modest amount.

In addition to the lines from the $\mathrm{SN}$, the spectrum also shows a number of interstellar absorption lines from the Milky Way and the host galaxy, shown in the lower part of Figure 9. In wavelength order we identify these as Si II $\lambda \lambda 1190.4,1193.3$, $1194.5,1197.4$, N I $\lambda \lambda 1199.6,1200.2$, Si III $\lambda 1206.5$, N v $\lambda \lambda 1238.8,1242.8$, Si II $\lambda \lambda 1260.4,1264.7$, O I $\lambda \lambda 1302.2,1304.9$, 1306.0, C II $\lambda \lambda 1334.5,1335.7$, Si IV $\lambda \lambda 1393.8,1402.8$, Si II $\lambda \lambda 1526.7,1533.4, \mathrm{C}$ IV $\lambda \lambda 1548.2,1550.8$, Fe II $\lambda 1608.5$, and Al II $\lambda 1670.8$. The lower signal-to-noise $(\mathrm{S} / \mathrm{N})$ and spectral resolution of the STIS spectra make interstellar medium (ISM) line identifications more difficult. We do, however, detect a number of strong lines: Fe II $\lambda \lambda 2344.2,2374.5,2382.8,2586.6$, 2600.2, 2607.9, Mg II $\lambda \lambda 2796.4$, and 2803.5. All of these lines are commonly seen in different directions of the Milky Way as well as in different galaxies.

To show the relative contributions from the UV and optical ranges, we show in Figure 11 the complete UVoptical spectrum at two epochs, one early and one very late. For the first epoch, this is a combination of the COS and STIS spectra from day 44 and an interpolation of the FAST spectra from day 32 and day 58 to this date. For the late spectrum, we exclude the COS spectrum because of the strong contamination of the continuum by the host galaxy by the large COS aperture. The emission lines in this spectrum are well defined and, with a few exceptions, free from contamination.

From Figure 11 we note the increasing importance of the UV at late phases, as was also concluded from Figure 3. This is similar to other Type IIn SNe, like SN 1995N (Fransson et al. 2002) and SN 1998S (Fransson et al. 2005), but is in contrast to Type IIP and Ibc SNe, which rapidly become faint in the UV. We 

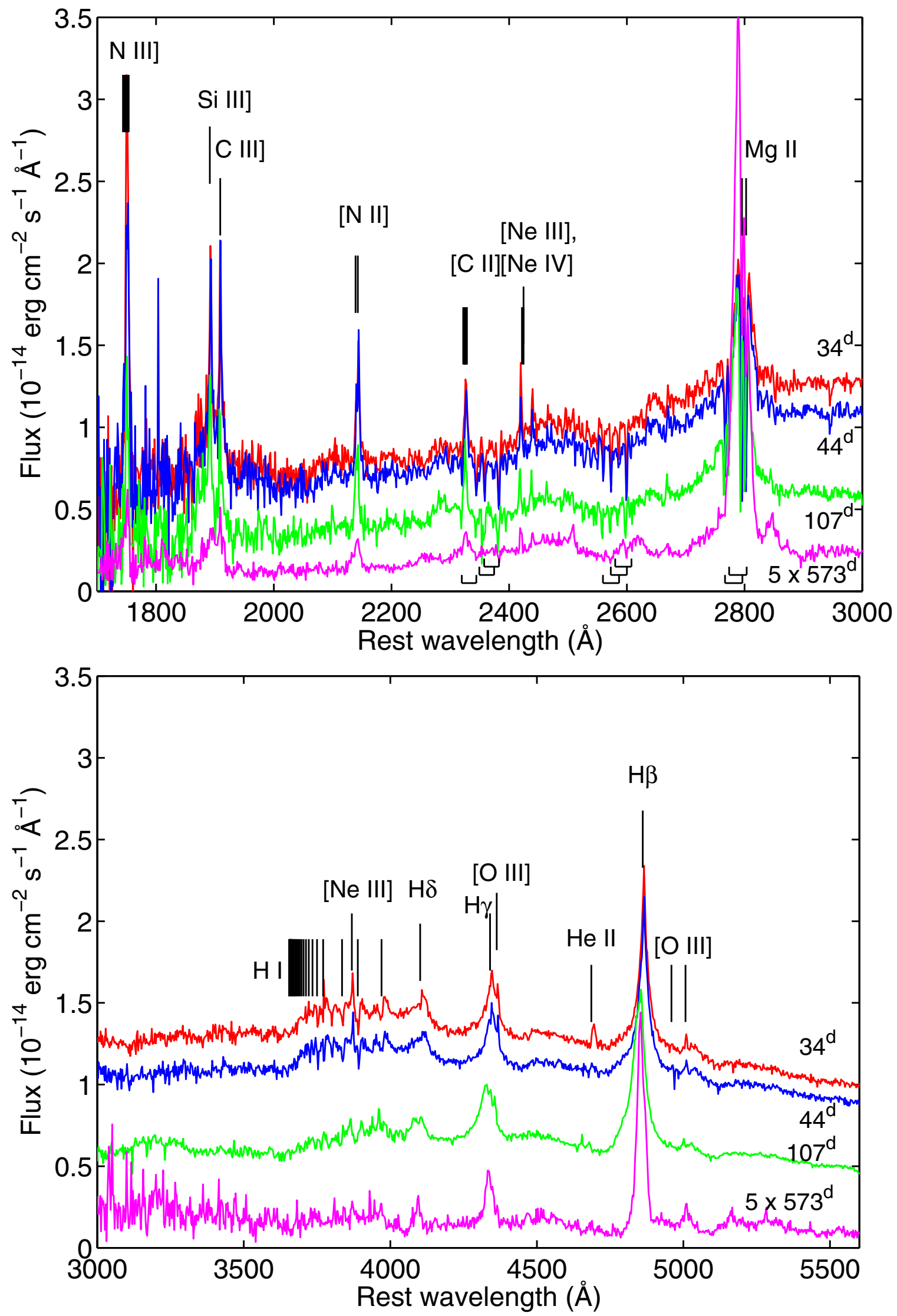

Figure 10. UV and optical STIS spectra from days 34 (red), 44 (blue), 107 (green), and 573 (magenta). The last spectrum has been multiplied by a factor of 5.0 for clarity. Note the strong [N II] and N III] lines in the UV spectrum, as well as the broad, symmetric line profiles for the strongest lines.

(A color version of this figure is available in the online journal.) 


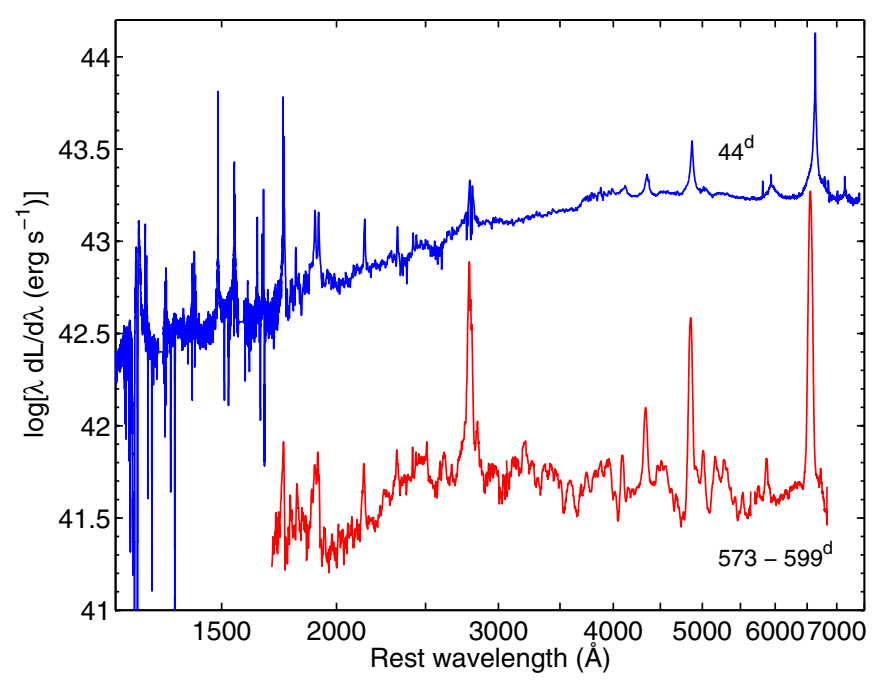

Figure 11. Combined UV and optical spectra from day 44 and days 573-599 from COS, STIS, and ground-based observations. For $\lambda>5700 \AA$ on day 44 , we use an interpolated spectrum from the FAST day 32 and day 58 observations. Both spectra are dereddened. We plot $\log \lambda d L / d \lambda$ against $\log \lambda$, which gives the luminosity distribution per wavelength decade.

(A color version of this figure is available in the online journal.)

also see a clear change in the character of the spectrum, from one dominated by a continuum at early epochs to one dominated by line emission at the last epochs.

\subsection{Line Profiles and Flux Evolution}

The medium-resolution optical spectra in Figure 8, as well as the high S/N COS spectrum in Figure 9, clearly show that there are two distinct velocity components in the strongest emission lines. This is apparent for the $\mathrm{H} \mathrm{I}$ and $\mathrm{Mg}$ II lines but also for highionization UV lines like C IV, N III-V, O III-IV, Si IV, and N V. The broad components extend to $\sim 2400-10,000 \mathrm{~km} \mathrm{~s}^{-1}$, and the narrow components have an order of magnitude lower velocities. The maximum velocity to where the broad component can be traced depends mainly on the flux of the line and the $\mathrm{S} / \mathrm{N}$ of the spectra. For both of these reasons, the $\mathrm{H} \alpha$ line displays the most extended wings. We now discuss the properties of these components in more detail.

\subsubsection{The Broad Component}

In Figure 12 we show the SN 2010jl spectral sequence of the $\mathrm{H} \alpha$ line from day 31 to day 1128 . The broad lines display a smooth, peaked profile, characteristic of electron scattering (Münch 1948; Auer \& van Blerkom 1972; Hillier 1991; Chugai 2001). At early times the lines are symmetric between the red and blue wings, and after $\sim 50$ days they display a pronounced bump to the blue. As we discuss in detail in Section 4.3, we interpret this as a result of a macroscopic velocity. We discuss (and reject) the alternative, in which dust absorption determines the line shape, in Section 4.3.

To calculate the flux of $\mathrm{H} \alpha, \mathrm{H} \beta$, and $\mathrm{H} \gamma$, we determine the continuum level on either side of the line between $-15,000$ and $-12,000 \mathrm{~km} \mathrm{~s}^{-1}$ and between 12,000 and $15,000 \mathrm{~km} \mathrm{~s}^{-1}$. A continuum level is then determined as a linear interpolation between these velocities and subtracted from the total flux. Figure 12 shows that this should be an accurate estimate.

In Figure 13, we show the $\mathrm{H} \alpha$ luminosity together with the $\mathrm{H} \alpha / \mathrm{H} \beta$ and $\mathrm{H} \beta / \mathrm{H} \gamma$ ratios. The dashed lines give polynomial least-squares fits to the data. In addition, we show the continuum

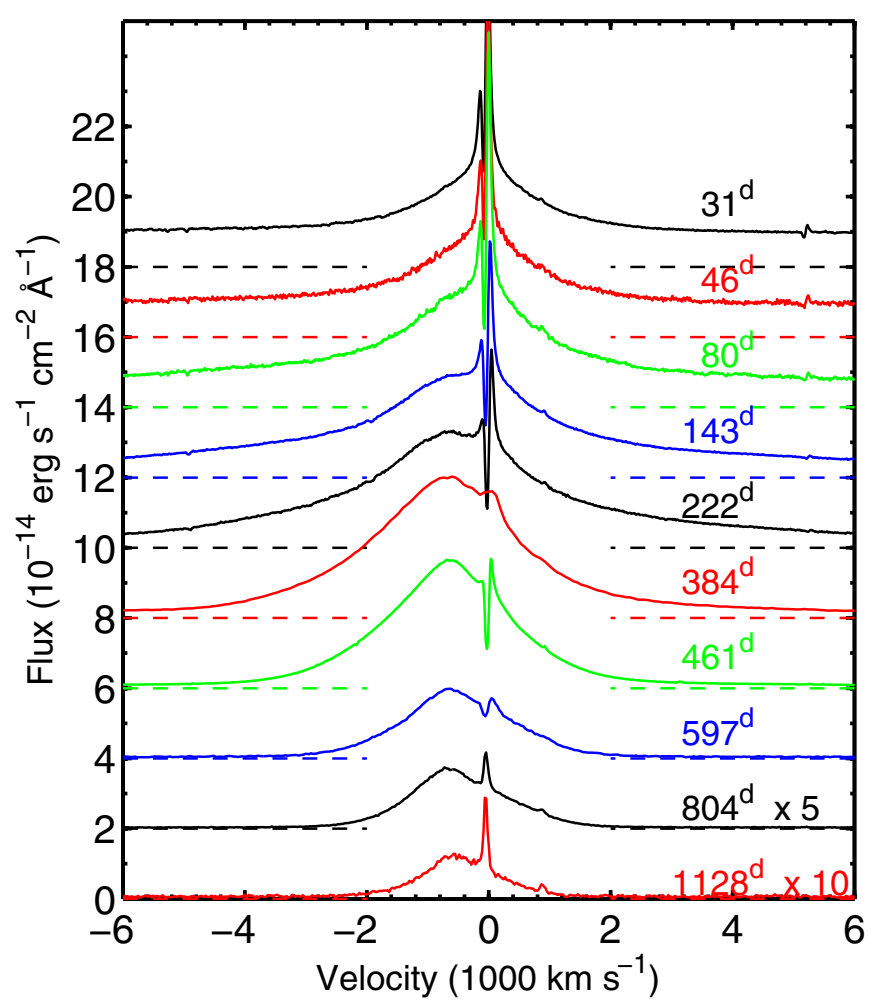

Figure 12. Evolution of the broad component of $\mathrm{H} \alpha$ from day 31 to day 1128 from NOT grism 17 (upper five panels), FAST (day 384), X-shooter (day 461), MDM (day 597), and MMT (days 804 and 1128). Note the evolution from a symmetric profile centered at $v=0$ to the late blueshifted line profile. The narrow P-Cygni component is smoothed out in the lower resolution day 384 FAST spectrum and partly also in the day 597 MDM spectrum. Each spectrum is shifted by $2 \times 10^{-14} \mathrm{erg} \mathrm{s}^{-1} \mathrm{~cm}^{-2} \AA^{-1}$. The dashed lines show the zero level for each date. For clarity, the day 804 spectrum has been multiplied by a factor of five and the day 1128 spectrum by a factor of 10 .

(A color version of this figure is available in the online journal.)

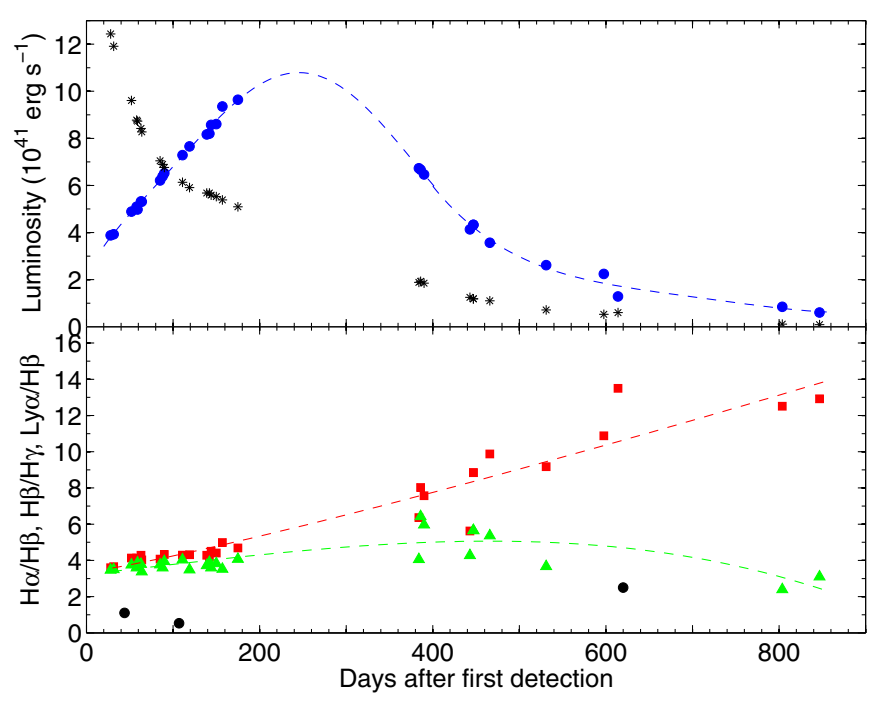

Figure 13. Upper panel: evolution of the continuum-subtracted flux of the broad $\mathrm{H} \alpha$ line within $\pm 10,000 \mathrm{~km} \mathrm{~s}^{-1}$ (blue dots) and the continuum flux in the same region (black asterisks). Lower panel: the $\mathrm{H} \alpha / \mathrm{H} \beta$ ratio (red squares) and the $\mathrm{H} \beta / \mathrm{H} \gamma$ ratio (green triangles) for the same period. The dashed lines are polynomial least-square fits to the respective data points. We have also added the $\mathrm{Ly} \alpha / \mathrm{H} \beta$ ratios for the three epochs where we have COS spectra (black dots). Luminosities and line ratios are corrected for reddening.

(A color version of this figure is available in the online journal.) 
flux in the same velocity interval, $\pm 10,000 \mathrm{~km} \mathrm{~s}^{-1}$, as the lines. From the figure we see that $\mathrm{H} \alpha$ increases by a factor of $\sim 2.5$ from day 29 to day 175 . After the first observational gap between $\sim 200$ days and $\sim 400$ days, the flux has dropped somewhat, and it then drops by another factor of $\sim 10$ from 400 to 850 days. From the polynomial fit it is likely that the maximum $\mathrm{H} \alpha$ luminosity occurred at $\sim 260$ days when the $\mathrm{SN}$ was close to the Sun. The bell-shaped light curve of the H $\alpha$ luminosity is in contrast to the continuum light curve in the same velocity region, shown as black dots, which decreases monotonically, and by a factor of $\sim 100$ from the first observations up to 800 days. The total luminosity (continuum plus $\mathrm{H} \alpha$ ) remains nearly constant up to $\sim 400$ days, after which it too drops. The increasing flux in $\mathrm{H} \alpha$ during the first $\sim 300$ days explains the flatter light curve of the $r^{\prime}$ band shown in Figure 2.

For the first $\sim 500$ days our $\mathrm{H} \alpha$ luminosity agrees well with the total luminosity from the "narrow" and "intermediate"components of Zhang et al. (2012). Their terminology is different from that used in this paper. Their "narrow" and "intermediate"components are both part of our "broad," and their data do not resolve what we refer to as the "narrow"component. Our light curve, however, covers approximately one more year, showing a continued decay of $\mathrm{H} \alpha$.

The $\mathrm{H} \alpha / \mathrm{H} \beta$ flux ratio increases steadily from $\sim 3.6$ initially to $10-15$ at $\sim 800$ days. The $\mathrm{H} \beta / \mathrm{H} \gamma$ ratio similarly increases from $\sim 3.4$ initially to $\sim 5.0$ at $\sim 400$ days, after which it decreases to $\sim 2.8$ at 800 days (Figure 13). For Balmer lines higher than $\mathrm{H} \delta$, the broad components blend together into a quasi-continuum, and the Balmer decrement for these is therefore difficult to determine.

The Ly $\alpha$ line, shown in Figure 14, is severely distorted by interstellar absorption lines both from the host galaxy and from the Milky Way. The broad Ly $\alpha$ absorption from the host galaxy makes it impossible to determine if any narrow emission is present. The red side of the broad component is relatively unaffected by absorptions and extends to $\sim 2460 \mathrm{~km} \mathrm{~s}^{-1}$. The blue side reaches into the damping profile of the host-galaxy absorption. However, a lower limit of $\sim 2300 \mathrm{~km} \mathrm{~s}^{-1}$ to the blue extent of the line can be determined. As is shown by the comparison with the $\mathrm{H} \beta$ line from the same-epoch STIS spectrum, the two lines have similar profiles in the velocity ranges where $\operatorname{Ly} \alpha$ is unaffected by absorptions. The lower flux of the Ly $\alpha$ line between $-2000 \mathrm{~km} \mathrm{~s}^{-1}$ and $-1000 \mathrm{~km} \mathrm{~s}^{-1}$ compared to the $\mathrm{H} \alpha$ and $\mathrm{H} \beta$ lines is caused by the overlapping ISM absorptions, as is the Si III $\lambda 1206.5$ line (Section 3.1). A similar reasoning applies to the other epochs, although we also observe a change in the ratio of the blue and red segments of the line from the first two epochs to the last. This is caused by the blueshift of the line, so more of the red side is absorbed by the host galaxy, and the blue region is increasing for the same reason.

In Section 3.1 we found that the damping wings of the Ly $\alpha$ absorption imply a value of $N_{\mathrm{H}} \sim(1.05 \pm 0.3) \times 10^{20} \mathrm{~cm}^{-2}$ for $b=10 \mathrm{~km} \mathrm{~s}^{-1}$ from the host galaxy. This means that the large column density derived from the Chandra observations, $\sim 10^{24} \mathrm{~cm}^{-2}$ (Chandra et al. 2012b), must be intrinsic to the $\mathrm{SN}$ itself or its CSM.

The ratios between the $\mathrm{Ly} \alpha, \mathrm{H} \beta$, and $\mathrm{H} \alpha$ lines are useful as indicators of the conditions in the broad-line region of active galactic nuclei (AGNs; e.g., Krolik \& McKee 1978; Fraser et al. 2013). Because of the broad absorptions, the total flux of the Ly $\alpha$ line cannot be directly determined. Instead we estimate the line ratio of this line to $\mathrm{H} \alpha$ and $\mathrm{H} \beta$ by first subtracting the
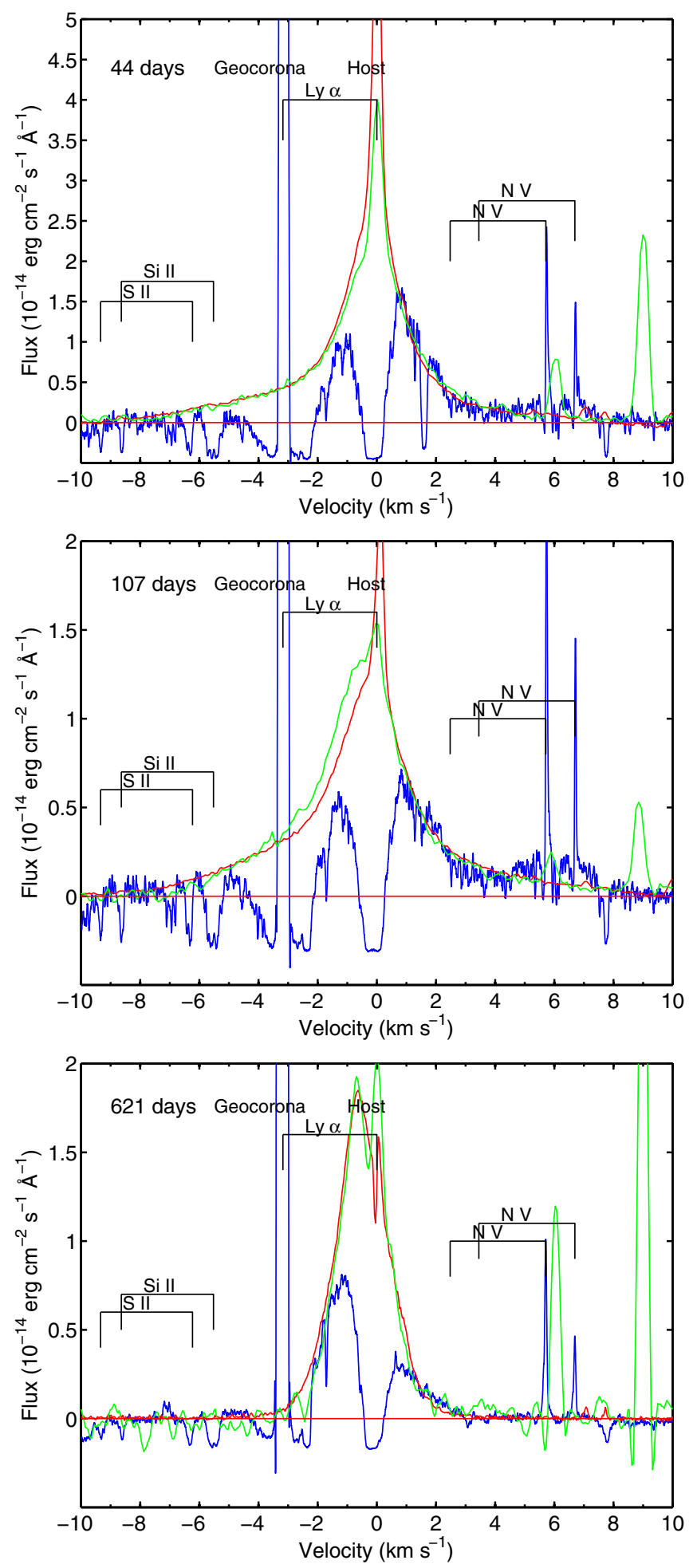

Figure 14. Ly $\alpha$ line profile (blue) from the COS spectra of days 44, 107, and 621 compared to the $\mathrm{H} \alpha$ (red) and $\mathrm{H} \beta$ (green) lines. The scale and continuum level of $\mathrm{H} \alpha$ and $\mathrm{H} \beta$ lines have been adjusted to agree with those of Ly $\alpha$. The positions of the N V emission lines are marked, as well as the strongest interstellar lines from the host galaxy and our Galaxy in the Ly $\alpha$ spectrum.

(A color version of this figure is available in the online journal.)

continuum and then scaling the line profiles so that these fit each other as well as possible for the parts of the lines unaffected by interstellar absorptions (see Figure 14). The scaling factor then gives the line ratio. 
Including reddening with $E_{\mathrm{B}-\mathrm{V}}=0.058$ (Section 3.1), we find for the first $\operatorname{COS}$ observation at day 44 $F(\mathrm{Ly} \alpha) / F(\mathrm{H} \beta)=1.1$ and $F(\mathrm{H} \alpha) / F(\mathrm{H} \beta)=2.8$, for day 107 $F(\mathrm{Ly} \alpha) / F(\mathrm{H} \beta)=0.53$ and $F(\mathrm{H} \alpha) / F(\mathrm{H} \beta)=4.1$, and for day $620 F(\mathrm{Ly} \alpha) / F(\mathrm{H} \beta)=2.5$ and $F(\mathrm{H} \alpha) / F(\mathrm{H} \beta)=9.0$. Because of the difficulty in the calibration of the COS spectra relative to the optical spectra, the uncertainty in the $\operatorname{Ly} \alpha / \mathrm{H} \beta$ ratio is large. We have plotted the $\mathrm{Ly} \alpha / \mathrm{H} \beta$ ratio in Figure 13 as black dots.

Although the uncertainties in the Ly $\alpha$ flux are large, we see from these ratios that the broad-line region has a Balmer decrement and a $\mathrm{Ly} \alpha / \mathrm{H} \alpha$ ratio very different from the recombination values. This is similar to what is found in the dense broad-line regions in AGNs, and it indicates a combination of high optical depths in the lines and high densities. Models show that column densities of $\gtrsim 10^{24} \mathrm{~cm}^{-2}$ in combination with high densities, $\gtrsim 5 \times 10^{8} \mathrm{~cm}^{-3}$, are needed to get these ratios (Krolik \& McKee 1978; Kwan 1984; Fraser et al. 2013). A flat X-ray spectrum also lowers the $\mathrm{Ly} \alpha / \mathrm{H} \alpha$ ratio and increases the $\mathrm{H} \alpha / \mathrm{H} \beta$ ratio (Kwan 1986). There is, however, a substantial degeneracy between the density, column density, and X-ray flux. In Section 4.7 we discuss this further based on real models.

Besides the $\mathrm{H}_{\mathrm{I}}$ lines, $\mathrm{N} v \lambda \lambda 1238.8,1242.8$, O I $\lambda \lambda 1302.2$ 1306.0, O IV] $\lambda \lambda 1397.2-1407.3+$ Si IV $\left.\lambda \lambda 1393.8,1402.8, N_{\text {IV }}\right]$ $\lambda 1486.5$, and C IV $\lambda \lambda 1548.2-1550.8$ also have broad components. This is probably also the case for He II $\lambda 1640.5, \mathrm{O}$ III] $\lambda \lambda 1660.8-1666.2, \mathrm{~N}$ III] $\lambda \lambda 1746.8-1754.0$, and Si III] $\lambda 1892.0 /$ $\mathrm{C}$ III] $\lambda 1908.7$, although the flux of the broad component is comparable to the $\mathrm{S} / \mathrm{N}$ for these lines.

In Figure 15, we compare the broad components from the strong $\mathrm{C}_{\mathrm{IV}}$ and $\mathrm{N}$ IV] lines with the profile of $\mathrm{H} \beta$. Although the $\mathrm{S} / \mathrm{N}$ in the COS observation is lower, we see that the profiles are basically identical, as expected for electron scattering from lines originating at similar depths.

Figure 16 shows the evolution of the most important broad UV lines from the COS and STIS observations. The scattering wings of both the $\mathrm{N}$ IV] and $\mathrm{C}$ IV lines get fainter between the first two observations at 44 and 107 days, opposite to the evolution of the $\mathrm{H} \alpha$ line. At 621 days the wings of the high-ionization lines have completely disappeared, in contrast to the Ly $\alpha$, O I $\lambda \lambda 1302.2-1306.0$, and Mg II lines, which all still have strong wings in the last HST observations. The evolution of the latter lines agrees more with $\mathrm{H} \alpha$.

\subsubsection{The Narrow Component}

As noted by Smith et al. (2011a), many of the optical lines display a narrow component, in most cases with a P-Cygni profile, as shown in Figure 8. In Figure 17 we show the narrow $\mathrm{H} \alpha$ line on a larger velocity scale from NOT/Grism17, TRES, $\mathrm{X}$-shooter, and MDM spectra. The peak of the line is close to zero velocity, and the red wing reaches $\sim 150 \mathrm{~km} \mathrm{~s}^{-1}$, as marked by the vertical lines. The blue wing is more complex, consisting of both an absorption reaching $\sim-105 \mathrm{~km} \mathrm{~s}^{-1}$ and a highvelocity emission reaching $\sim-250 \mathrm{~km} \mathrm{~s}^{-1}$. These velocities do not change appreciably with time, although the emission components get weaker.

The emission wing on the blue side of the P-Cygni absorption is likely to be caused by electron scattering in the CSM of the SN. Examples of this are known for WR stars, where Hillier (1991, see his Figure 8) calculated the P-Cygni profile, including electron scattering in the line-forming zone, and obtained a line profile similar to that seen for $\mathrm{H} \alpha$. The expansion velocity of the
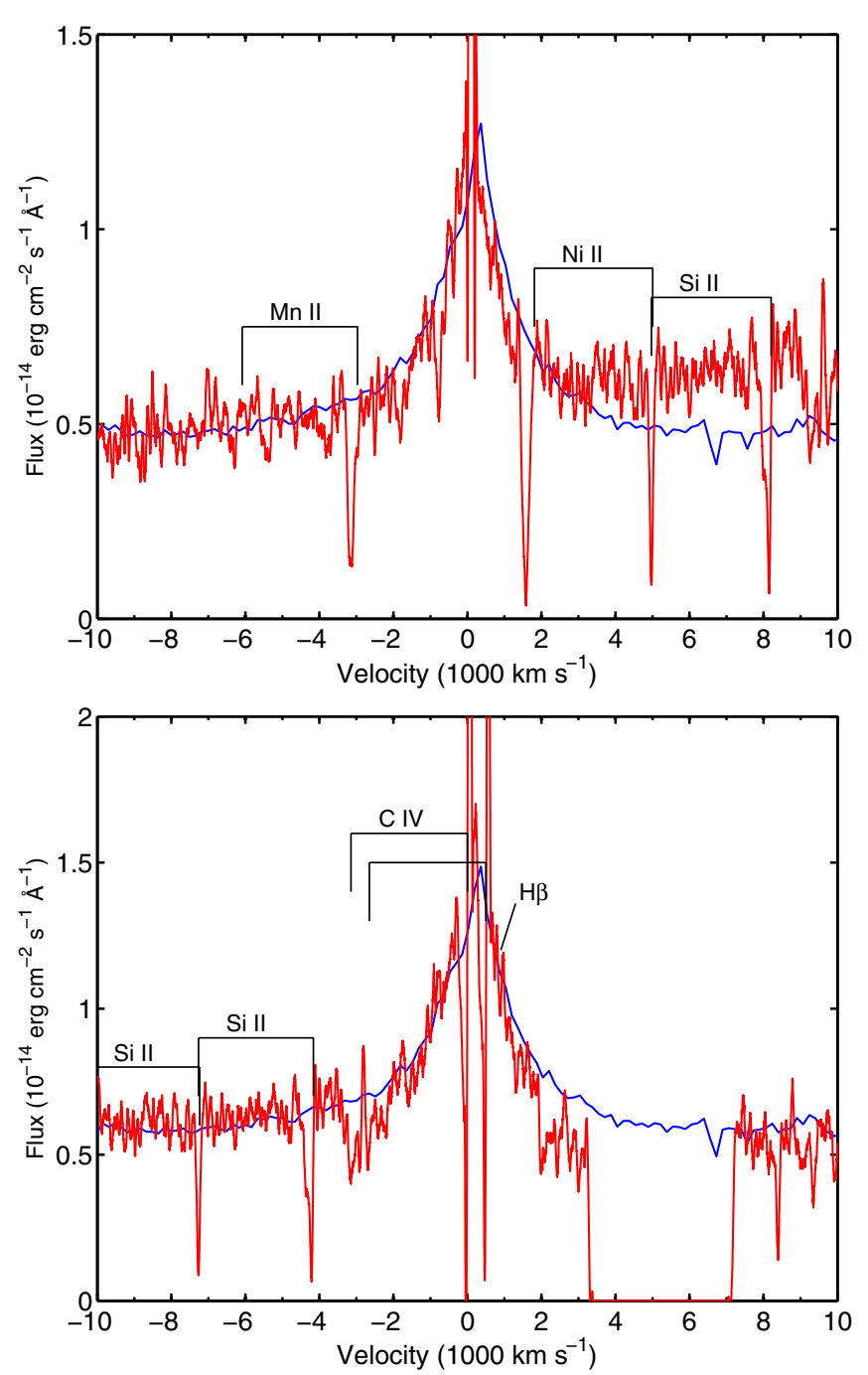

Figure 15. Comparison of the day $44 \mathrm{~N} \mathrm{IV]} \mathrm{(top)} \mathrm{and} \mathrm{C} \mathrm{IV} \mathrm{(bottom)} \mathrm{lines} \mathrm{with}$ the $\mathrm{H} \beta$ line profile (blue). The positions of some of the interstellar absorption lines from the host and our Galaxy are marked.

(A color version of this figure is available in the online journal.)

CSM should therefore be $\sim-105 \mathrm{~km} \mathrm{~s}^{-1}$, rather than the higher velocity characteristic of the blue wing. This also shows that the electron scattering optical depth is not negligible in the CSM of the SN.

In Figure 18 we show the net flux of the narrow $\mathrm{H} \alpha$ line from our highest dispersion spectra. For the narrow component we have interpolated the broad line flux on each side of the line $\left(-250 \mathrm{~km} \mathrm{~s}^{-1}\right.$ and $200 \mathrm{~km} \mathrm{~s}^{-1}$, respectively) and then calculated the net emission above this level. In the day 461 spectrum, the P-Cygni absorption is stronger than the emission, and the luminosity of the line is consequently negative.

The main change with time of the narrow component is a decrease of the flux (and equivalent width) of the line, from a net emission line to a line with zero or even negative equivalent width. When we compare the broad and narrow emission we see that they evolve independently from each other. The narrow component is in fact more correlated to the continuum luminosity than to the broad $\mathrm{H} \alpha$.

Of the He I lines the following lines are detected: $\lambda 3889$ (blended with H 8-2), $\lambda 3965$ (P-Cygni), $\lambda 4471$ (P-Cygni), $\lambda 4713$ (only emission), $\lambda 4922$ (probable), $\lambda 5016$ (P-Cygni), 


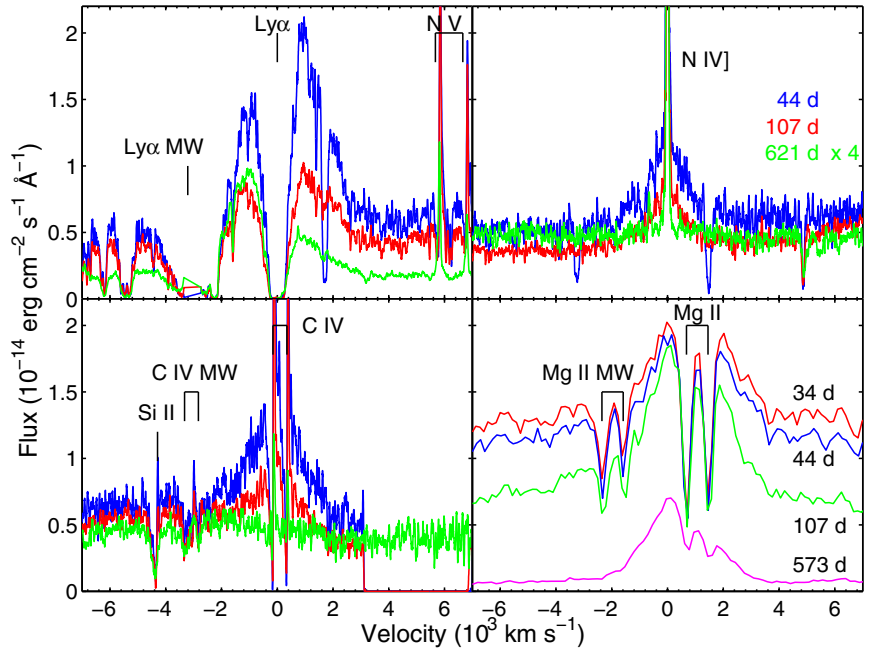

Figure 16. Evolution of the profiles of the Ly $\left.\alpha, \mathrm{N}_{\mathrm{IV}}\right], \mathrm{C}_{\mathrm{IV}}$, and $\mathrm{Mg}$ II lines. The $\mathrm{Ly} \alpha, \mathrm{N}$ IV], and C IV spectra are from $\mathrm{COS}$, and the $\mathrm{Mg}$ II profile is from the lower resolution STIS spectra. Note that for clarity the flux of the COS spectrum for day 621 has been multiplied by a factor of 4.0 .

(A color version of this figure is available in the online journal.)

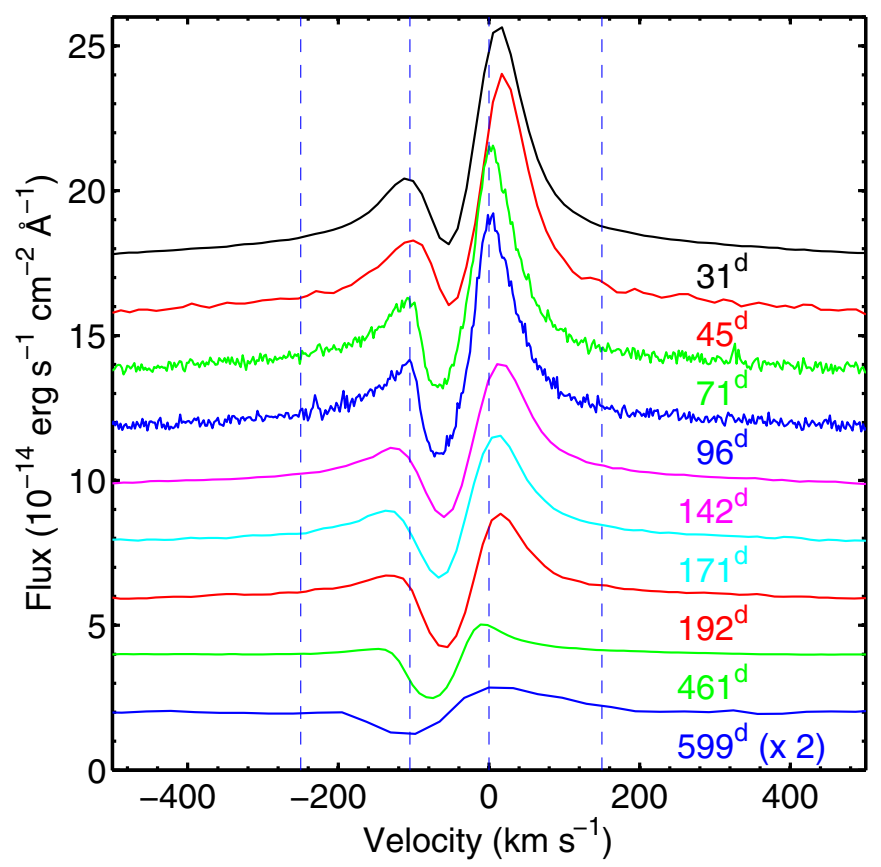

Figure 17. Narrow component of $\mathrm{H} \alpha$ from day 31 to day 599. The continuumlevel has been set as the average flux between 300 and $500 \mathrm{~km} \mathrm{~s}^{-1}$, and each spectrum has then been shifted by $2 \times 10^{-14} \mathrm{erg} \mathrm{s}^{-1} \mathrm{~cm}^{-2} \mathrm{~s}^{-1} \AA^{-1}$ relative to the previous. The vertical dashed lines are at $-250,-105,0$, and $+150 \mathrm{~km} \mathrm{~s}^{-1}$. The day 96 and day 142 spectra are from TRES, the day 461 from X-shooter, and the day 599 spectrum from MDM, and the others come from NOT/grism 17. The flux of the day 599 spectrum has been multiplied by a factor of two.

(A color version of this figure is available in the online journal.)

$\lambda 5876$ (P-Cygni), $\lambda 6678$ (P-Cygni), and $\lambda 7065$ (pure emission). Although some of these are seen in H II regions, the fact that P-Cygni profiles are observed for most of them shows that they originate in the SN environment. Additional He I lines are present in the NIR (Section 3.4.3).

Figure 19 shows a sample of the most important other narrow emission lines in the UV and optical ranges on a velocity

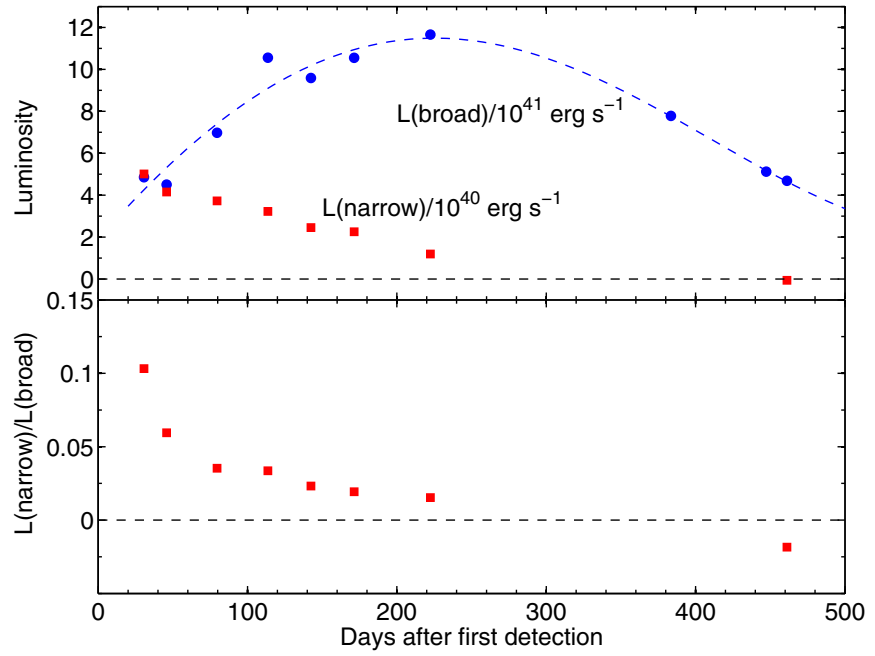

Figure 18. Upper panel: the luminosity of the broad and narrow components of $\mathrm{H} \alpha$ from day 31 to day 461. Only spectra with resolution higher than FWHM $1.2 \AA$ have been included for the narrow component. The day 96 and day 142 spectra are from TRES, the day 461 from X-shooter, and the others come from NOT/grism 17. For the narrow line we give the net luminosity in the P-Cygni line. The last measurement at 461 days has a stronger absorption component than emission component, and the luminosity is therefore negative. Note that the luminosity scale of the narrow and broad lines are different by a factor of 10. Lower panel: ratio of these luminosities.

(A color version of this figure is available in the online journal.)

scale for the day 44 and day 621 spectra. When comparing the line profiles of these lines, one has to take into account the absorption of the UV resonance lines from the host galaxy at redshift $3207 \mathrm{~km} \mathrm{~s}^{-1}$ and the Milky Way. An important case is the $\mathrm{C}$ IV $\lambda \lambda 1548.2,1550.8$ doublet. The velocity separation of the $\lambda 1550.8$ component relative to the $\lambda 1548.2$ component corresponds to $500 \mathrm{~km} \mathrm{~s}^{-1}$. The absorption from the Milky Way does not interfere with the $\lambda 1548.2$ line below $2700 \mathrm{~km} \mathrm{~s}^{-1}$. Absorption from the host may, however, be important. Typical rotational velocities are $100-200 \mathrm{~km} \mathrm{~s}^{-1}$, and this may well interfere with both the absorption and emission components, making a determination of the expansion velocity difficult for these lines.

When we compare the different lines in Figure 19, we see that the absorptions of the OI $\lambda 1302.2$, Si IV $\lambda 1393.8$, and the CIV $\lambda 1548.2$ lines all are considerably wider than the intercombination lines. The strong N IV] $\lambda 1486.5$ line has a FWHM of $\sim 50 \mathrm{~km} \mathrm{~s}^{-1}$ and extends to $\sim 100 \mathrm{~km} \mathrm{~s}^{-1}$ to the red side and $\sim 80 \mathrm{~km} \mathrm{~s}^{-1}$ to the blue. These velocities are similar to the red emission component of the $\mathrm{C}$ IV lines but are considerably less than the $\mathrm{C}$ IV absorption. Similar widths are found for the $\mathrm{O}$ III] $\lambda 1666.2$ and He II $\lambda 1640.4$ lines, although the $\mathrm{S} / \mathrm{N}$ is lower for these lines. In contrast, the velocity of the blue edge of the C IV $\lambda 1548.2$ absorption corresponds to $\sim-280 \mathrm{~km} \mathrm{~s}^{-1}$. The extent of the strong emission component to the red is, however, only $\sim 100 \mathrm{~km} \mathrm{~s}^{-1}$, although it is difficult to determine this accurately.

The higher velocity of the absorption of the C IV $\lambda \lambda 1548.2,1550.8$ lines probably originates in the host-galaxy ISM or possibly in higher velocity CSM gas of low density whose emission is too faint to be seen. This is also consistent with the other resonance lines. For the O I $\lambda 1302.2$ line one can even distinguish a separation between the P-Cygni absorption at $\sim 100 \mathrm{~km} \mathrm{~s}^{-1}$ and the higher absorption component. 

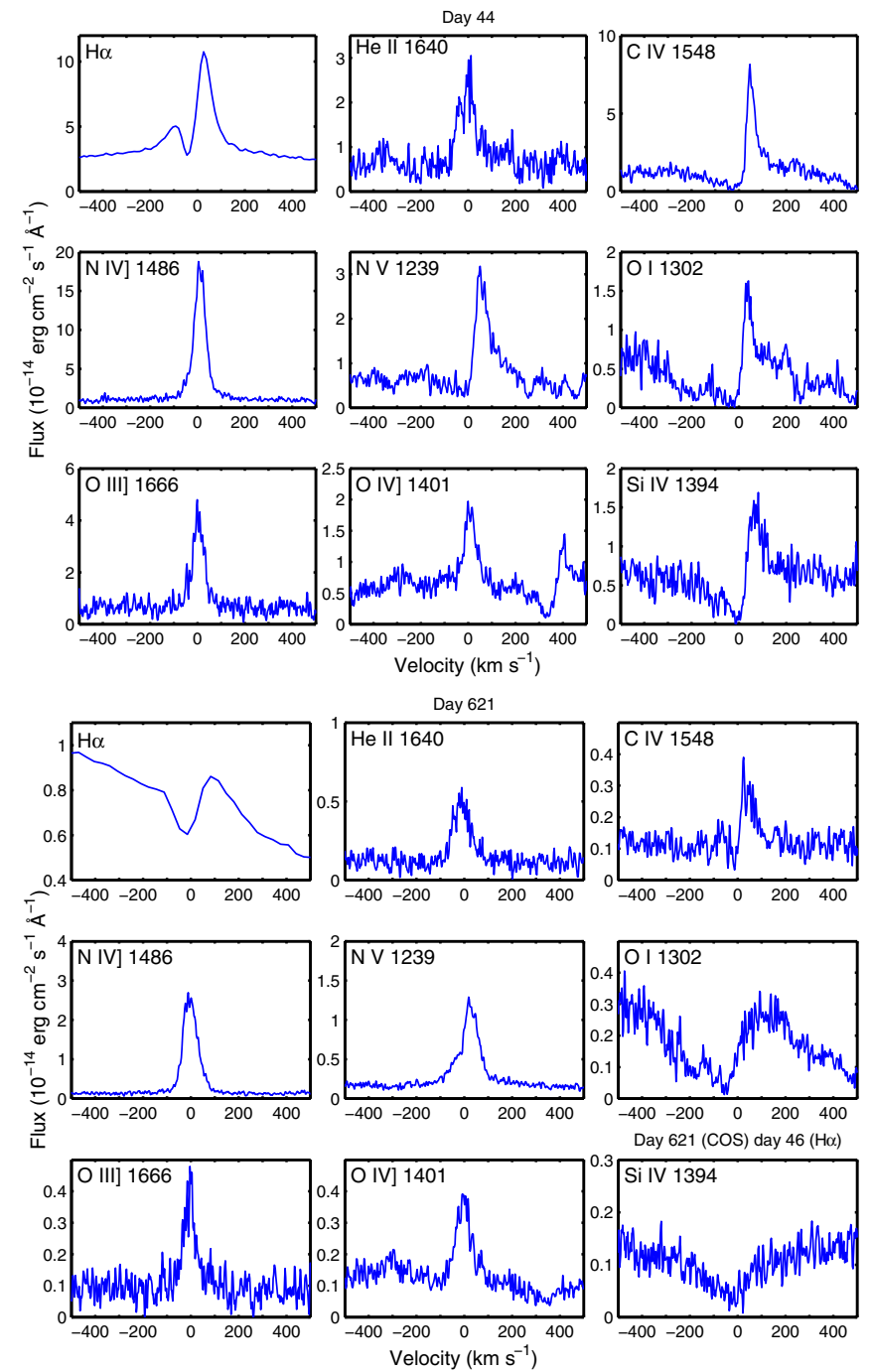

Figure 19. Central, low-velocity region of the line profiles of the $\mathrm{H} \alpha$, $\mathrm{He}$ II, Civ, Niv], N v, O I, O III], O IV], and Si IV lines on a velocity scale from day 44 (upper three rows) and day 621 (lower three rows). The $\mathrm{H} \alpha$ spectra are from days 46 and 597, respectively.

(A color version of this figure is available in the online journal.)

When we compare the line profiles of the day 44 and day 621 spectra, we do not see any clear evolution in velocity widths. The velocities of the high-ionization narrow UV lines are similar to the optical lines, i.e., $\sim 100 \mathrm{~km} \mathrm{~s}^{-1}$.

The fact that the Balmer and the He I lines have P-Cygni absorptions shows that the population of these excited levels is high even at $\sim 600$ days. This in turn argues for a high density, $\gtrsim 10^{8} \mathrm{~cm}^{-3}$, in the line-forming region (Section 4.7). In Section 3.5 we discuss other independent density determinations that indicate high-density circumstellar gas.

Tables 6 and 7 give fluxes of the identified narrow UV lines from the STIS and COS spectra. To measure the fluxes of the different lines, we first determine the continuum level on both sides of the line and fit this to a polynomial, which is subtracted from the total flux in the line. This introduces uncertainties in both the continuum level and the extent of the line, which is most serious for the lower resolution STIS spectrum, which also has a lower $\mathrm{S} / \mathrm{N}$. In addition, the low spectral resolution of STIS also has the consequence that multiplets, like $\mathrm{N}$ III] $\lambda \lambda 1746.8-1754.0$, are not resolved. To complicate things further, several lines, like the N III], Si III], and $\mathrm{C}$ III] lines, sit on top of broad electron-scattering profiles (see below). The higher spectral resolution COS spectra are less sensitive to these effects. Unfortunately, this setting of COS does not cover the $\mathrm{C}$ III] lines.

A further complication, when using these lines for diagnostic purposes, is that all of the resonance lines in the UV have P-Cygni components. The photons, which give rise to the absorption component, scattered out of the line of sight (LOS) of the observer are partly compensated for by those scattered into the LOS from the area of the wind outside of the projected photosphere. The net emission in a line from the CSM comes from the emission component plus the scattered photons. The fraction of the photons from the back of the SN and that are in the LOS of the optically thick ejecta do not contribute to the emission. An upper limit to this contribution is the absorbed flux relative to the continuum in the absorption component. The exact fraction depends on the emissivity as function of the radius from the photosphere. For this reason we list in Table 7 both the flux in the emission component and the flux deficit in the absorption component for the COS spectra.

An inspection of Table 6, as well as Figure 10, shows that between the first two epochs there is relatively little evolution of the flux of the narrow lines in the STIS range, although the continuum has decreased somewhat. At 107 days the fluxes of both continuum and lines have, however, decreased by a factor of $\sim 2$. The same trends can be seen in the COS spectra (Table 7 and Figure 9). The decrease in the narrow line fluxes correlates with the broad component of the high-ionization UV lines (Figure 16). In the last STIS spectrum at 573 days, both lines and continuum have dropped by a factor of $\sim 10$, although the $\mathrm{Mg}$ II $\lambda \lambda 2796,2804$ only drops by a factor of $\sim 3$.

\subsubsection{The $X$-shooter Spectrum at 461 Days}

We also obtained one medium/high-resolution X-shooter spectrum from the VLT for 2012 January 14 (day 461), shown in Figure 20. Because of the higher spectral resolution and NIR coverage, we discuss this spectrum separately.

Starting with the continuum, we note the excess in the NIR with a maximum at $\sim 1.5 \mu \mathrm{m}$. This NIR excess agrees well with the rise in the $J, H$, and $K_{s}$ bands we see in the photometry in Figure 2 later than $\sim 400$ days and is most likely caused by hot dust emission; its origin will be discussed in Section 4.2. We fit this well with a blackbody spectrum at a temperature of $\sim 1870 \mathrm{~K}$. The corresponding continuum in the optical is fitted with a temperature of $\sim 9000 \mathrm{~K}$. The value for the dust temperature differs by $\sim 8 \%$ from that obtained from the photometry in Table 5, $2045 \mathrm{~K}$. This gives an estimate of the errors in this parameter from the errors in the photometry, spectral calibration, and fitting errors. Because of the many lines in the optical region, the error in the photospheric temperature is at least as large as this, although here they happen to be very close.

In the NIR the most interesting lines are the strong broad and narrow components of $\mathrm{He} \mathrm{I} \lambda 10,830$, as well as $\mathrm{Pa} \alpha, \mathrm{Pa} \beta$, and $\mathrm{Pa} \gamma$. The He I $\lambda 10,830$ confirms the identification of the He I $\lambda 5876$ line in the optical. The $\lambda 2.0581 \mu \mathrm{m}$ line is, however, only seen as a narrow absorption.

We identify the broad, asymmetric feature with a peak at $\sim 8435 \AA$ with O I $\lambda 8446$. The extended red wing is a blend of the Ca II triplet $\lambda \lambda 8498.0,8542.1,8662.1$ and higher members 
Table 6

STIS Line Catalog

\begin{tabular}{|c|c|c|c|c|c|c|}
\hline \multirow[t]{2}{*}{ Species } & \multirow{2}{*}{$\begin{array}{c}\lambda_{\mathrm{vac}} \\
(\AA)\end{array}$} & \multicolumn{4}{|c|}{$\begin{array}{c}\text { Line Flux } \\
\left(10^{-14} \mathrm{erg} \mathrm{cm}^{-2} \mathrm{~s}^{-1}\right)\end{array}$} & \multirow[t]{2}{*}{ Notes } \\
\hline & & day 34 & day 44 & day 107 & day 573 & \\
\hline O III] & $1660.81-1666.15$ & 2.8: & 2.4: & & & At wavelength limit \\
\hline N III] & $1746.82-1754.00$ & $18.4 \pm 2.0$ & $13.5 \pm 2.0$ & $5.88 \pm 0.8$ & $0.60 \pm 0.10$ & \\
\hline Si III] & $1882.71-1896.64$ & $8.80 \pm 0.6$ & $9.12 \pm 1.5$ & $7.64 \pm 0.5$ & $0.51 \pm 0.15$ & \\
\hline C III] & $1906.68-1909.60$ & $8.50 \pm 1.2$ & $8.40 \pm 1.2$ & $5.20 \pm 1.0$ & $0.56 \pm 0.15$ & \\
\hline $\mathrm{C}$ II] & $2324.21-2328.83$ & $2.46 \pm 0.3$ & $2.75 \pm 0.2$ & $2.12 \pm 0.2$ & $0.37 \pm 0.10$ & \\
\hline Mg II & 2796.34-2803.52 & $14.5 \pm 0.5$ & $25.6 \pm 1.0$ & $38.4 \pm 2.0$ & $12.8 \pm 2.00$ & Broad component \\
\hline [Ne III] & 3869.85 & $1.44 \pm 0.1$ & $1.37 \pm 0.2$ & $0.77 \pm 0.3$ & $\ldots$ & \\
\hline $\mathrm{HI}_{\mathrm{I}}$ & 4102.89 & $7.73 \pm 0.3$ & $6.72 \pm 3.0$ & $5.43 \pm 1.8$ & $0.57 \pm 0.20$ & Broad component \\
\hline $\mathrm{HI}$ & 4341.68 & $18.6 \pm 0.9$ & $16.2 \pm 2.5$ & $19.1 \pm 1.5$ & $2.39 \pm 0.30$ & Broad component \\
\hline [O III $]$ & 4364.45 & $0.85 \pm 0.2$ & $0.56 \pm 0.3$ & $0.50 \pm 0.15$ & $0.12 \pm 0.03$ & \\
\hline He II & 4687.02 & $1.45 \pm 0.2$ & $0.57 \pm 0.3$ & - & $\ldots$ & Marginal detection \\
\hline [O III $]$ & 5008.24 & $0.79 \pm 0.2$ & $0.70 \pm 0.3$ & $0.30 \pm 0.15$ & $0.23 \pm 0.10$ & \\
\hline
\end{tabular}

Table 7

SN2010j1 COS Line Catalog

\begin{tabular}{|c|c|c|c|c|c|}
\hline \multirow[t]{2}{*}{ Species } & \multirow{2}{*}{$\begin{array}{c}\lambda_{\mathrm{vac}} \\
(\AA)\end{array}$} & \multicolumn{3}{|c|}{$\begin{array}{c}\text { Line Flux } \\
\left(10^{-15} \mathrm{erg} \mathrm{cm}^{-2} \mathrm{~s}^{-1}\right)\end{array}$} & \multirow[t]{2}{*}{ Notes } \\
\hline & & day 44 & day 107 & day 620 & \\
\hline $\mathrm{Nv}$ & 1238.82 & $-0.76 \pm 0.3$ & $-0.44 \pm 0.2$ & $0.08 \pm 0.05$ & P-Cygni abs \\
\hline $\mathrm{Nv}$ & 1238.82 & $7.38 \pm 0.5$ & $6.64 \pm 0.5$ & $4.24 \pm 0.10$ & \\
\hline $\mathrm{Nv}$ & 1242.80 & $-0.68 \pm 0.2$ & $-0.35 \pm 0.2$ & $-0.64 \pm 0.20$ & P-Cygni abs \\
\hline $\mathrm{Nv}$ & 1242.80 & $3.25 \pm 0.5$ & $2.96 \pm 0.4$ & $1.68 \pm 0.08$ & \\
\hline O I & 1302.17 & $-2.40 \pm 1.0$ & $-2.05 \pm 0.9$ & $-2.01 \pm$ & P-Cygni abs \\
\hline O I & 1302.17 & $1.71 \pm 0.6$ & $1.33 \pm 0.6$ & $\ldots$ & \\
\hline OI & 1304.86 & $\ldots$ & $\ldots$ & $-1.42 \pm$ & complex P-Cygni abs \\
\hline $\mathrm{O}_{\mathrm{I}}$ & 1304.86 & $2.64 \pm 0.3$ & $2.05 \pm 0.3$ & $\ldots$ & \\
\hline $\mathrm{O}_{\mathrm{I}}$ & 1306.03 & $1.55 \pm 0.7$ & $1.84 \pm 0.3$ & $\ldots$ & \\
\hline Si II & 1309.28 & $-0.61 \pm 0.3$ & $-0.57 \pm 0.2$ & & P-Cygni abs \\
\hline Si II & 1309.28 & $0.37 \pm 0.5$ & $0.07 \pm 0.4$ & & \\
\hline Si IV & 1393.76 & $-2.21 \pm 0.9$ & $-1.66 \pm 0.5$ & $-1.23 \pm 0.15$ & P-Cygni abs \\
\hline Si IV & 1393.76 & $3.36 \pm 1.6$ & $1.04 \pm 0.4$ & $\ldots$ & \\
\hline O IV] & 1397.20 & $0.53 \pm 0.2$ & $0.39 \pm 0.2$ & $\ldots$ & \\
\hline O IV] & 1399.78 & $0.92 \pm 0.2$ & $0.77 \pm 0.2$ & $0.22 \pm 0.10$ & \\
\hline O IV & 1401.16 & $4.04 \pm 0.2$ & $3.59 \pm 0.3$ & $0.59 \pm 0.20$ & \\
\hline Si IV & 1402.77 & $-1.53 \pm 0.5$ & $-0.99 \pm 0.3$ & $-1.03 \pm$ & P-Cygni abs \\
\hline Si IV & 1402.77 & $2.16 \pm 0.9$ & $0.48 \pm 0.3$ & $\ldots$ & \\
\hline O IV] & 1404.81 & $2.20 \pm 0.5$ & $2.52 \pm 0.3$ & $0.56 \pm 0.15$ & \\
\hline S IV] & 1406.00 & $1.06 \pm 0.2$ & $0.72 \pm 0.2$ & $\ldots$ & \\
\hline O IV] & 1407.38 & $0.40 \pm 0.2$ & $0.57 \pm 0.2$ & $\ldots$ & \\
\hline N IV] & 1483.32 & $\ldots$ & $\cdots$ & $0.25 \pm 0.05$ & \\
\hline N IV] & 1486.50 & $54.7 \pm 1.8$ & $51.7 \pm 1.5$ & $9.61 \pm 0.10$ & \\
\hline CIV & 1548.19 & $-4.25 \pm 1.7$ & $-2.85 \pm 0.5$ & $\ldots$ & P-Cygni abs \\
\hline CIV & 1548.19 & $15.9 \pm 1.0$ & $8.98 \pm 0.8$ & $0.58 \pm 0.07$ & \\
\hline CIV & 1550.77 & $-4.36 \pm 0.4$ & $-2.54 \pm 0.5$ & $\ldots$ & P-Cygni abs \\
\hline CIV & 1550.77 & $8.90 \pm 2.0$ & $4.90 \pm 0.7$ & $0.31 \pm 0.05$ & \\
\hline Ne IV] & $1601.5 ?$ & $\ldots$ & $\ldots$ & $0.65 \pm 0.07$ & \\
\hline He II & 1640.40 & $8.55 \pm 0.3$ & $8.64 \pm 0.3$ & $1.81 \pm 0.10$ & \\
\hline O III] & 1660.81 & $4.96 \pm 0.2$ & $2.88 \pm 0.2$ & $0.53 \pm 0.10$ & \\
\hline O III] & 1666.15 & $12.6 \pm 0.3$ & $9.64 \pm 0.3$ & $1.48 \pm 0.10$ & \\
\hline $\mathrm{N}$ III] & 1746.82 & $1.53 \pm 0.5$ & $0.73 \pm 0.3$ & $\ldots$ & \\
\hline $\mathrm{N}$ III] & 1748.65 & $5.51 \pm 0.7$ & $3.89 \pm 0.3$ & $\ldots$ & \\
\hline N III] & 1749.67 & $42.9 \pm 0.8$ & $27.1 \pm 0.7$ & $1.62 \pm 0.07$ & \\
\hline $\mathrm{N}$ III] & 1752.16 & $20.8 \pm 0.7$ & $15.1 \pm 0.4$ & $0.86 \pm 0.05$ & \\
\hline N III] & 1754.00 & $7.44 \pm 0.7$ & $6.47 \pm 0.3$ & $0.23 \pm 0.05$ & \\
\hline
\end{tabular}

of the Paschen series $(n=12-15)$. The identification with the O I $\lambda 8446$ line is supported by the presence of the O I $1.129 \mu \mathrm{m}$ line. These lines are likely to be a result of $\operatorname{Ly} \beta$ fluorescence with $\mathrm{O}$, where the upper level of the $1.129 \mu \mathrm{m}$ line is pumped by
$\operatorname{Ly} \beta$ and then decays into the $1.129 \mu \mathrm{m}$ and $\lambda 8446$ lines (Grandi 1980). The relative flux of these lines agrees well with what is expected, although blending of the O I $\lambda 8446$ line prevents a detailed comparison. 


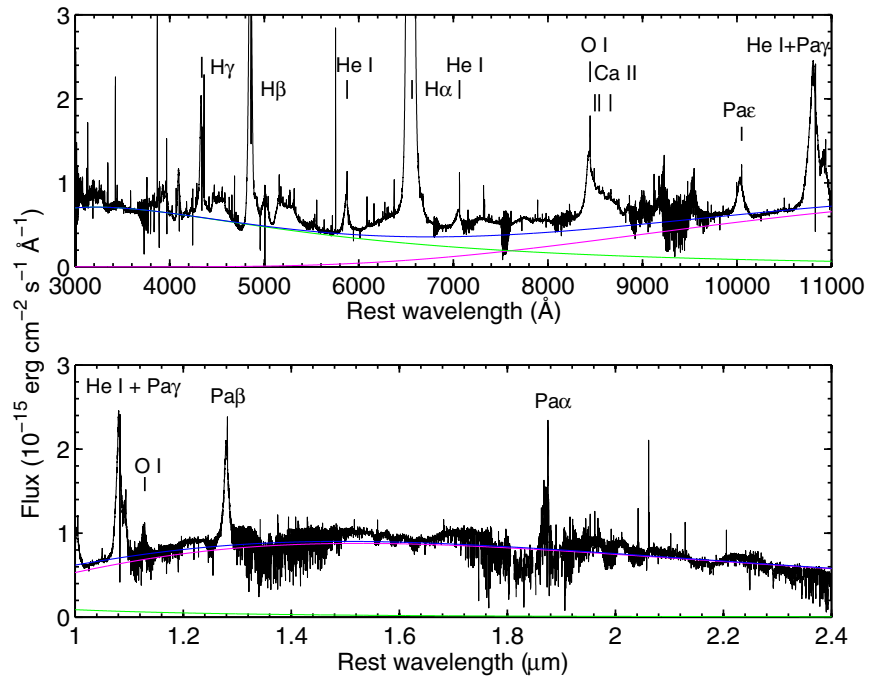

Figure 20. X-shooter spectrum from 2012 January 14 (day 461). The continuum has been fit with a two-component blackbody with temperatures of $9000 \mathrm{~K}$ and $1870 \mathrm{~K}$, respectively. Note the many narrow lines from the circumstellar gas, as well as the strong, broad He I and H I lines in the NIR.

(A color version of this figure is available in the online journal.)

O I $\lambda 8446$ was also seen in the Type IIn SN $1995 \mathrm{G}$ (Pastorello et al. 2002), although in this case no NIR spectrum exists. There is also a line consistent with O I $\lambda 8446$ in the $S N 1995 \mathrm{~N}$ spectrum in Fransson et al. (2002), although this was identified as mainly Fe II $\lambda 8451$. In the case of SN 2010jl there are, however, no lines corresponding to Fe II $\lambda \lambda 9071,9128,9177$, which would be expected to accompany the Fe II $\lambda 8451$ line.

The high resolution in combination with high $\mathrm{S} / \mathrm{N}$ allows us to identify a large number of weak, narrow emission lines not seen in the other lower $\mathrm{S} / \mathrm{N}$ spectra that cast additional light on the CSM. In Figure 21 we show a close-up of the optical range with some of the most important lines marked.

The most interesting parts of these lines are the large range of ionization stages of forbidden lines from [Fe V-XIV].
The [Fe VII] lines serve as diagnostics of the density and the excitation conditions in the CSM and are discussed in Section 3.5.

Of the coronal lines, [Fex] $\lambda 6374.5$ is strong, as is [Fe XI] $\lambda 7891.8$, but [Fe XIV] $\lambda 5302.9$ is only barely seen as a weak line. [Fe XIII] $\lambda 10,746$ is not seen, but it sits on the strong wing of $\mathrm{He} \mathrm{I} \lambda 10,830$. Other high-ionization lines include [Ne v] $\lambda \lambda 3345.8,3425.9,[\mathrm{Ca} \mathrm{v}] \lambda 6086.8,[\mathrm{Ar} \mathrm{x}] \lambda 5533.2$, and a weak [Ar XIV] $\lambda 4412.6$. The presence of these lines confirms the high state of ionization in the CSM of this SN.

In these high $\mathrm{S} / \mathrm{N}$ spectra, the Balmer series can be traced up to $n=33$ ( $\lambda 3659.4)$ with strong absorption components but only weak P-Cygni emission. The Paschen series is seen from $\mathrm{Pa} \alpha$ to $n=17$ ( $\lambda 8467.3)$. In contrast to the Balmer lines, these lines are all in pure emission. $\mathrm{Br} \gamma$ is also seen in emission.

\subsection{Density Diagnostics of the Narrow Lines}

Using fluxes from the previous section, we use the forbidden and intercombination lines in the UV and optical as diagnostics of the density.

The ratios of the $\left.\mathrm{N}_{\mathrm{III}}\right] \lambda \lambda 1746.8,1748.7,1749.7,1752.2$, 1754.0 lines are sensitive to the electron density (Keenan et al. 1994). In particular, the $\lambda \lambda 1752.2 / 1749.7$ ratio is $0.55-0.58$ in the range $10^{5}-10^{8} \mathrm{~cm}^{-3}$. Above this density the ratio decreases to become 0.20 at $10^{10} \mathrm{~cm}^{-3}$. The $\lambda \lambda 1754.0 / 1749.7$ ratio has a similar behavior. Unfortunately, the $\lambda 1754.0$ line is at the very boundary of the wavelength range of the G160M grating, and its flux is uncertain. The $\lambda 1748.7$ and $\lambda 1754.0$ lines have the same upper level with transition rates within a few percent of each other, so we use the $\lambda \lambda 1748.7 / 1749.7$ ratio instead of the $\lambda \lambda 1754.0 / 1749.7$ ratio for the diagnostics.

Figure 22 shows a fit of these lines for different electron densities in the range $10^{7}-10^{10} \mathrm{~cm}^{-3}$ for the day $44 \mathrm{COS}$ spectrum. The fits show that (with the exception of the $\lambda 1754.0$ line) all lines agree with an electron density in the range $10^{5}-10^{8} \mathrm{~cm}^{-3}$. Higher values give too low a value for the $\lambda \lambda 1752.2 / 1749.7$ ratio. We have also examined the day 107

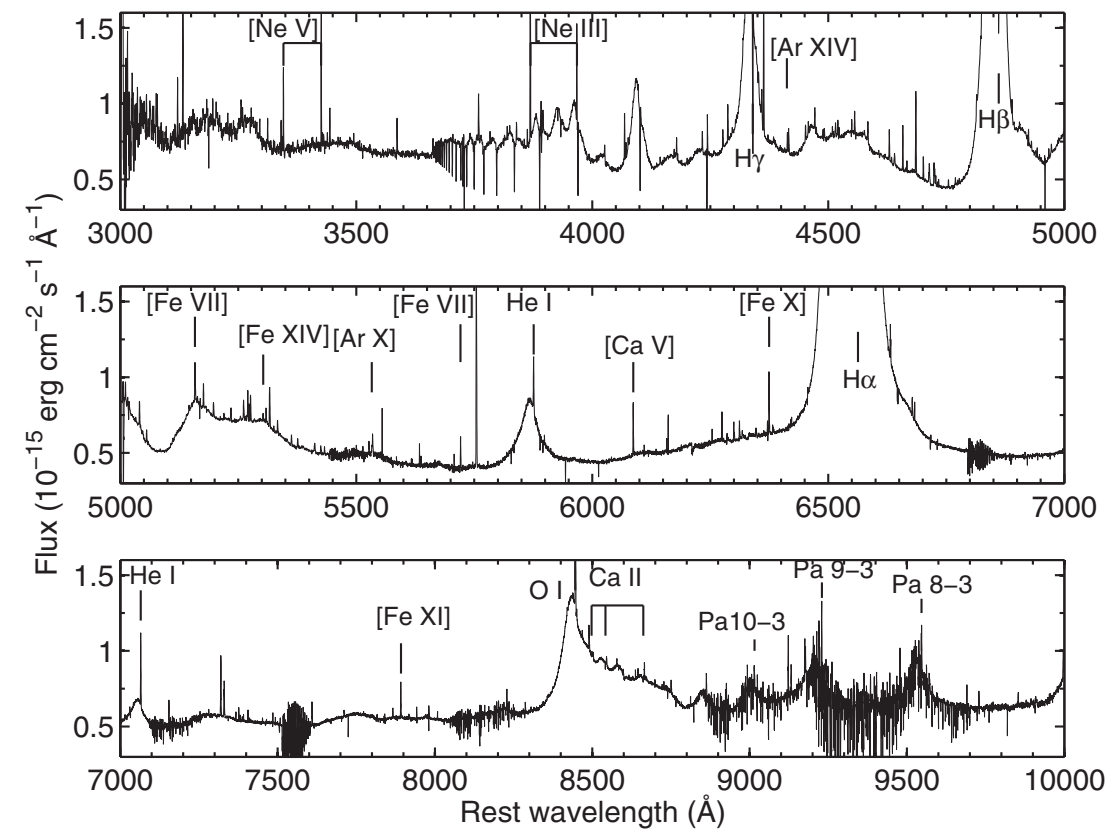

Figure 21. Optical part of the X-shooter spectrum from 2012 January 14 (day 461). A number of the most important lines from the CSM have been marked. 


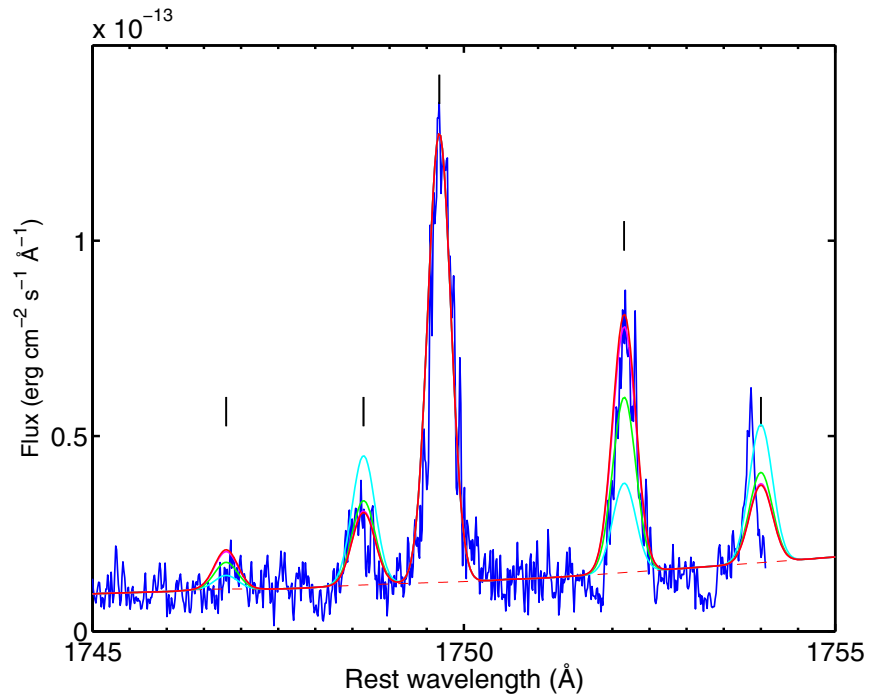

Figure 22. Fits to the $\mathrm{N}$ III] $\lambda \lambda 1746.8,1748.7,1749.7,1752.2,1754.0$ multiplet (marked by the vertical lines) from the COS spectrum from day 44 . The red line is for $10^{7} \mathrm{~cm}^{-3}$, the magenta for $10^{8} \mathrm{~cm}^{-3}$, the green for $10^{9} \mathrm{~cm}^{-3}$, and the cyan for $10^{10} \mathrm{~cm}^{-3}$. The dashed red line marks the continuum. The dip at $1753.3 \AA$ is an interstellar absorption line.

(A color version of this figure is available in the online journal.)

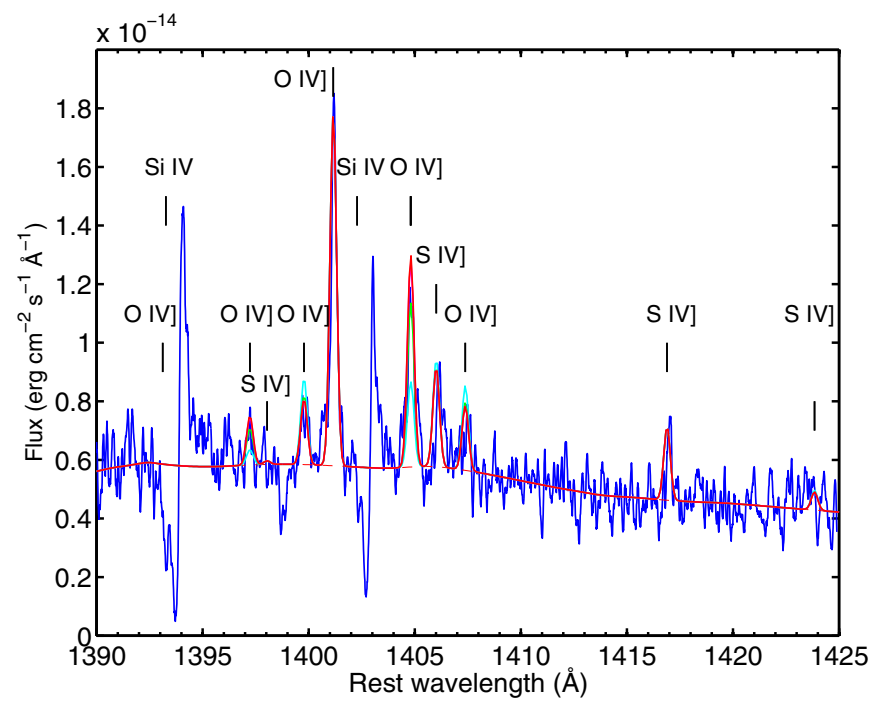

Figure 23. Same as Figure 22 for the O IV] $\lambda \lambda 1393.1,1397.2,1399.8,1401.2$, 1404.8, 1407.4 and S IV] $\lambda \lambda 1398.0,1404.8,1406.0,1416.9,1423.8$ multiplets. The red line is for $10^{7} \mathrm{~cm}^{-3}$, the magenta for $10^{8} \mathrm{~cm}^{-3}$, the green for $10^{9} \mathrm{~cm}^{-3}$, and the cyan for $10^{10} \mathrm{~cm}^{-3}$. The solid red line marks the continuum. We have not attempted a fit of the Si IV $\lambda \lambda 1393.3,1402.3$ P-Cygni lines.

(A color version of this figure is available in the online journal.)

and day 621 COS spectra and find essentially the same density constraint, although the $\mathrm{S} / \mathrm{N}$ is somewhat lower.

Figure 23 shows a similar analysis of the O IV] $\lambda \lambda 1393.1,1397.2,1399.8,1401.2,1404.8,1407.4$ and S IV] $\lambda \lambda 1398.0,1404.8,1406.0,1416.9,1423.8$ multiplets. As the red curve shows, the lines are fit with the same density interval, $10^{5}-10^{8} \mathrm{~cm}^{-3}$, as the $\mathrm{N} \mathrm{III]} \mathrm{lines.} \mathrm{This} \mathrm{is} \mathrm{not} \mathrm{surprising} \mathrm{because}$ these ions are expected to arise in the same region.

The ratio of the N IV] lines at $1482.9 \AA$ and $1486.1 \AA$ is sensitive to density in the range $10^{4}-10^{8} \mathrm{~cm}^{-3}$ (Keenan et al. 1995), with a ratio of $\sim 1.6$ below $10^{4} \mathrm{~cm}^{-3}$ and decreasing above this density. From the 2012 June 20 spectrum we estimate a $\lambda \lambda 1482.9 / 1486.1$ flux ratio of $0.029 \pm 0.05$. From Keenan

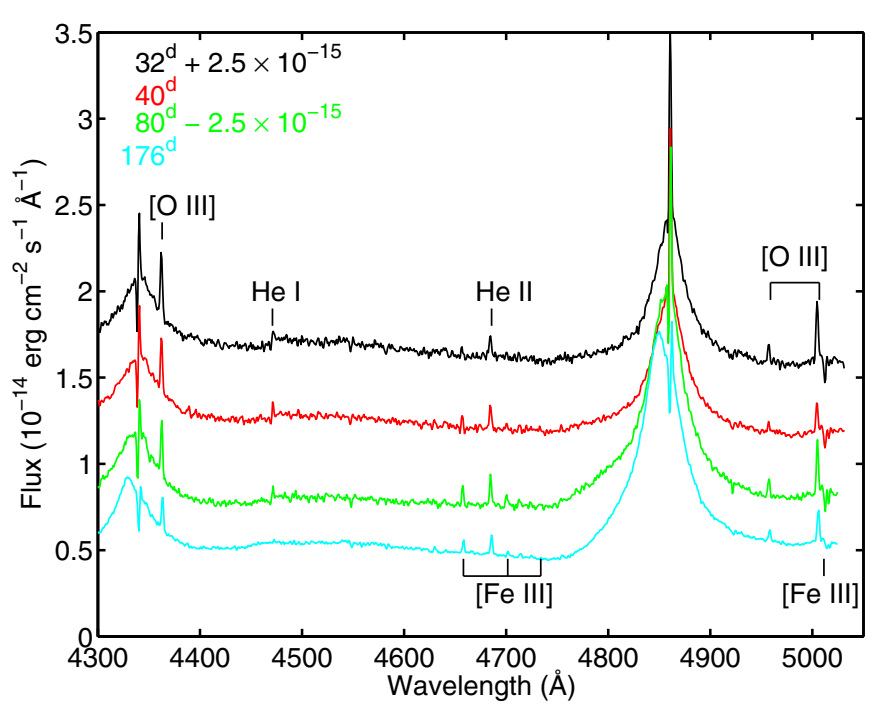

Figure 24. [O III] and [Fe III] region from observations with NOT from day 32 to day 176. Note the strong narrow [O III] $\lambda 4363$ line compared to the $\lambda 5007$ line. For clarity, the day 32 and day 80 spectra have been shifted in flux by the amount indicated in the figure.

(A color version of this figure is available in the online journal.)

et al. (1995, their Figure 1) we find that this corresponds to an electron density $(3-6) \times 10^{6} \mathrm{~cm}^{-3}$ for temperatures in the range $10^{4}-2 \times 10^{4} \mathrm{~K}$.

The O III] $\lambda \lambda 1660.8,1666.2$, and [O III] $\lambda \lambda 4363.2,4958.9$, 5007.0 lines are especially useful as density and temperature diagnostics Crawford et al. (2000). Figure 10 shows the [O III] $\lambda \lambda 4363.2,4958.9,5007.0$ region for the two first STIS spectra, and all three [O III] lines are easily identified. This is even clearer in the ground-based spectra in Figure 24. The first thing to note is the very strong $\lambda 4363$ line, which is usually faint compared to the $\lambda \lambda 4949,5007$ lines. Typical [O III] $\lambda \lambda 5007 / 4363$ ratios for $\mathrm{H}$ II regions are $\gtrsim 50$ (Osterbrock \& Ferland 2006). Pilyugin $\&$ Thuan (2007) determine [O III] $\lambda \lambda 4959,5007 / 4363$ ratios of 166 and 140 for UGC 5189 at different radii, which is much larger than the ratio observed for SN 2010jl and strongly argues for a circumstellar origin of these lines.

In Figure 25 we give the [O III] $\lambda \lambda 5007 / 4363$ ratio and the [O III] $\lambda 4363$ flux for the different dates for selected mediumresolution spectra with NOT and MMT (2010 November 15), as well as the STIS observations. The limited spectral resolution, as well as the $\mathrm{S} / \mathrm{N}$, makes the fluxes of the STIS spectra uncertain. They do, however, have the advantage of minimizing the background contamination of especially the $\lambda 5007$ line.

We note that the flux of the $\lambda 4363$ line shows a smooth decay by a factor of $\sim 2$ during the period. However, the $\lambda \lambda 5007 / 4363$ ratio varies by a large factor from observation to observation. The reason for this is that the $\lambda 5007$ line is severely affected by nearby $\mathrm{H}$ II regions in some of the ground-based observations. Observations with poor seeing $\left(\gtrsim 1^{\prime \prime}\right)$ therefore have a much larger $\lambda 5007$ flux than the ones with good seeing. This is also consistent with the STIS observations, which, in spite of a low $\mathrm{S} / \mathrm{N}$, show a low $\lambda 5007$ flux and a low $\lambda \lambda 5007 / 4363$ ratio. Because of the high $\lambda \lambda 5007 / 4363$ ratio in the nearby H II regions (see above), the $\lambda 4363$ line is only marginally affected by this. For the nebular analysis below we only use the lowest $\lambda \lambda 5007 / 4363$ ratios, which are in the range $0.5-0.8$.

The region of O III] $\lambda \lambda 1660.8,1666.2$ is noisy in the STIS spectra, but it does show a clear line above the noise (Figure 10). The flux of the line is consequently uncertain. However, the 


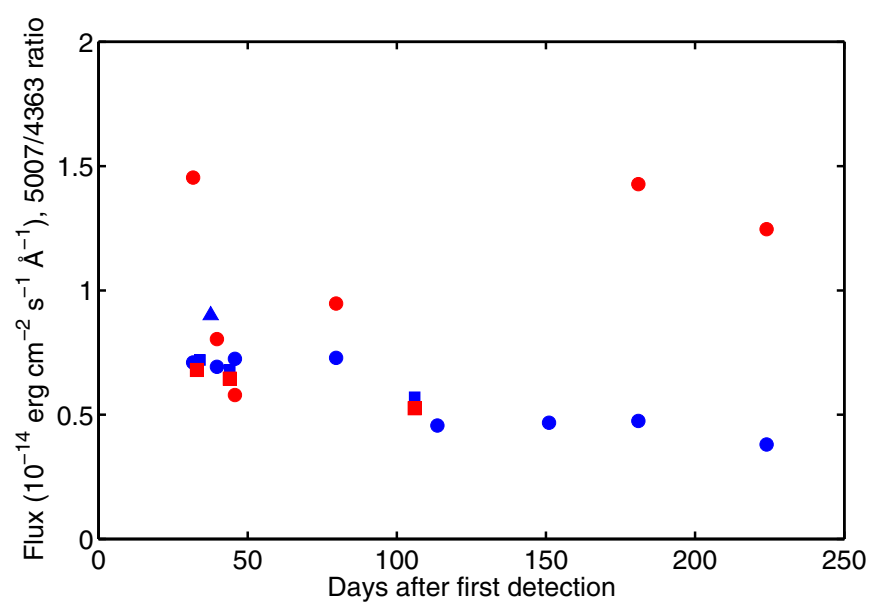

Figure 25. Flux of the narrow [O III $\lambda 4363$ line (blue) and the [O III] $\lambda \lambda 5007 / 4363$ ratio (red). Observations with STIS are marked with squares, MMT with triangles, and NOT/grism 16 with circles. The errors in the $\lambda 4363$ fluxes are $\sim 25 \%$, and the ground-based $\mathrm{O}$ III] $\lambda \lambda 5007 / 4363$ ratios are systematically overestimated because of background contamination from nearby $\mathrm{H}$ II regions. The observations with the best seeing give the lowest line ratios, as is confirmed with the STIS observations, although the S/N of these are lower. (A color version of this figure is available in the online journal.)

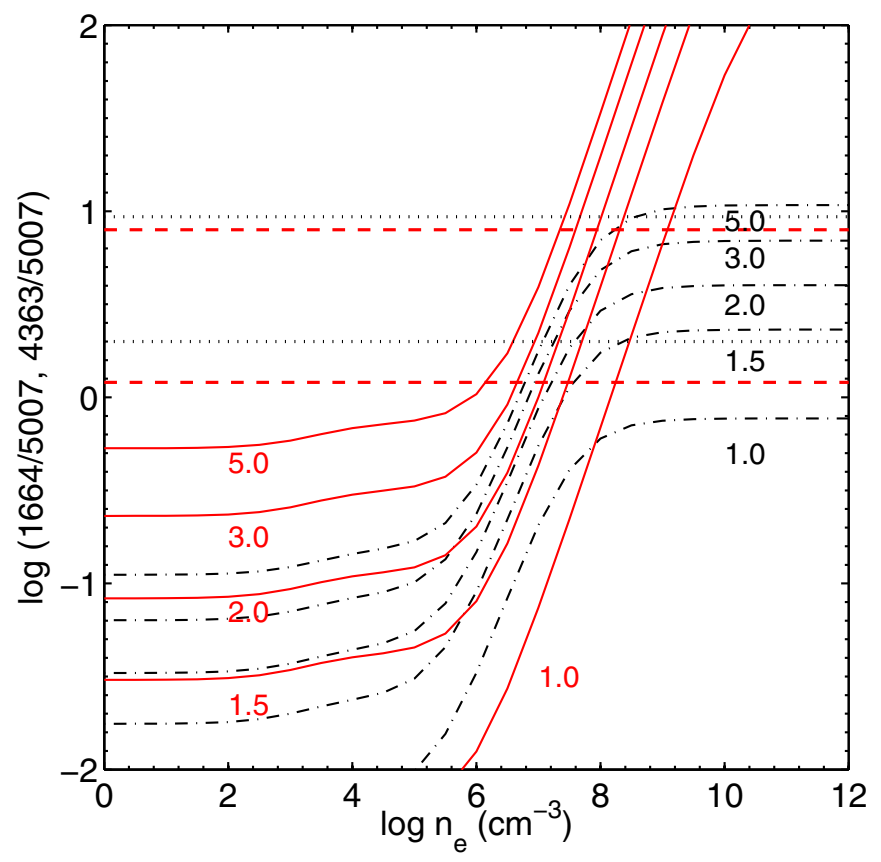

Figure 26. [O III] $\lambda \lambda 4363 / 5007$ (black dash-dotted lines) and $\lambda \lambda 1666 / 5007$ (red solid lines) ratios as a function of density and temperature (in $10^{4} \mathrm{~K}$ ). The corresponding observed ranges are plotted as horizontal dotted lines for the $\lambda \lambda 4363 / 5007$ ratio and dashed lines for the $\lambda \lambda 1666 / 5007$ ratio.

(A color version of this figure is available in the online journal.)

fluxes in the COS and STIS spectra are consistent with each other for the dates of the two COS observations. The observation from day 44 gives a total $\mathrm{O}$ III] $\lambda \lambda 1661+1666$ flux of $1.8 \times$ $10^{-14} \mathrm{erg} \mathrm{s}^{-1}$, close to that of the STIS observation for the same date. We conclude that the O III] $\lambda \lambda 1661+1664$ flux is accurate to at least $\sim 50 \%$. The expected line ratio of $\mathrm{O}$ III] $\lambda 1666.2 / \lambda 1660.8$ is 2.5 , consistent with the measured ratio of 2.3 from COS. As a very conservative estimate we take $\mathrm{O}$ III] $\lambda \lambda(1661+1666) / 5007=1.2-8$.

Figure 26 shows the [O III] $\lambda \lambda 4363 / 5007$ and $\lambda \lambda 1666 / 5007$ ratios as a function of density and temperature. We have also plotted the observed ranges of these ratios as horizontal lines in the figure. The atomic data are taken from Crawford et al. (2000) and Palay et al. (2012). From this figure we see that for reasonable temperatures, i.e., $\lesssim 50,000 \mathrm{~K}$, a $\lambda \lambda 5007 / 4363$ ratio in the range $0.5-0.8$ requires an electron density $\gtrsim 3 \times$ $10^{6} \mathrm{~cm}^{-3}$. Further, the $\lambda \lambda 1664 / 5007$ ratio results in a range $3 \times$ $10^{6}-10^{9} \mathrm{~cm}^{-3}$ for $T_{\mathrm{e}}=(1-3) \times 10^{4} \mathrm{~K}$. A higher reddening than we have assumed would only increase this range marginally.

We identify faint but clear narrow lines of [Fe III] at $4657 \pm 1 \AA$ and $4701 \pm 1 \AA$ in the high S/N NOT spectra (Figure 24). These lines can be seen at all epochs with approximately the same fluxes. In addition, there may also be a weak line at $\sim 5010 \AA$, but this is disturbed by the [O III] $\lambda 5007$ line, as well as noise. These lines are interesting in that they can provide some independent diagnostics of the narrow line gas. Keenan et al. (1993) find that the [Fe III] $\lambda \lambda 4702 / 4658$ ratio varies from $\sim 0.30$ for $n_{\mathrm{e}} \lesssim 10^{3} \mathrm{~cm}^{-3}$ to $0.46-0.52$ for $n_{\mathrm{e}} \gtrsim 10^{5} \mathrm{~cm}^{-3}$. The observed ratio is $\sim 0.5$, in agreement with the high-density limit. The $\lambda \lambda 4881 / 4658$ ratio increases from $\sim 0.2$ for $n_{\mathrm{e}} \approx 10^{2} \mathrm{~cm}^{-3}$ to $\sim 0.5$ for $n_{\mathrm{e}} \approx 10^{5} \mathrm{~cm}^{-3}$; at higher densities it rapidly decreases to $\lesssim 0.1$. There is no sign of the $\lambda 4881$ line in the spectra, consistent with a density $n_{\mathrm{e}} \gtrsim 10^{5} \mathrm{~cm}^{-3}$. The $\lambda 5011.3$ line is, on the other hand, probably present. Together, these lines are consistent with $n_{\mathrm{e}} \gtrsim 10^{6} \mathrm{~cm}^{-3}$.

The final diagnostics come from the [Fe VII] lines in the $\mathrm{X}$-shooter spectrum from day 461 (Figure 21). The [Fe VII] $\lambda \lambda 4988.6 / 5720.7$ ratio is 0.16 . Using the diagnostic diagram from Keenan et al. (2001), we find that this corresponds to an electron density of $\sim 5 \times 10^{5} \mathrm{~cm}^{-3}$. We note, however, that the derived density is sensitive to the uncertain collision strengths, as is discussed in Keenan et al. (2001).

Summarizing these different density diagnostics, i.e., the $\mathrm{N}$ III], Niv], [O III], O IV], [Fe III] and [Fe VII] ratios, as well as the presence of P-Cygni absorptions in the Balmer lines, we find that these lines indicate a density $3 \times 10^{6}-10^{8} \mathrm{~cm}^{-3}$ for the region emitting the narrow lines in SN 2010jl.

\subsection{CNO Abundances}

The strong [N II], N III], N IV], and N v lines in the STIS and COS spectra (Figures 9 and 10) indicate a nitrogen enrichment in the narrow-line region of the CSM of the SN.

To estimate the relative $\mathrm{CNO}$ abundances, we note that for a photoionized plasma the ionization zones of $\mathrm{N}$ III and $\mathrm{C}$ III coincide closely with each other, and similarly for the C IV, N IV, and O III zones (Kallman \& McCray 1982, their Figure 6(a)). The Nv and O IV zones overlap, but not to the same extent as the lower ionization ions. In addition, the temperature in these zones is nearly constant. Using the line fluxes for these ions allows us to derive the relative ionic abundances and, with some assumptions, elemental abundances. Because the excitation energies are similar, the line ratios are insensitive to the temperature for reasonable ranges, $(1-3) \times 10^{4} \mathrm{~K}$. Here we use $T_{\mathrm{e}}=2 \times 10^{4} \mathrm{~K}$. They do depend on the electron density, but this is mainly important above $\sim 10^{9} \mathrm{~cm}^{-3}$, where the lines are suppressed, and densities this high were excluded through the analysis in the previous section. The narrow range of wavelengths also makes the results insensitive to the reddening.

The most interesting lines for the CNO analysis are $\mathrm{N}$ IV] $\lambda 1486.5, \mathrm{C}$ IV $\lambda \lambda 1548.2,1550.8, \mathrm{O}$ III] $\lambda \lambda 1660.8,1666.2, \mathrm{~N}$ III] $\lambda \lambda 1746.8-1754.0$, and $\left.\mathrm{C}_{\mathrm{III}}\right] \lambda 1908.7$. With the exception of the $\mathrm{C}$ III] lines, all are within the range of our COS spectra, and accurate relative fluxes can be derived (within a few percent). The C III] line, however, poses a special problem because of 
Table 8

Observed and Derived Ionic Abundances

\begin{tabular}{|c|c|c|c|}
\hline Date & $\mathrm{N}$ III/C III & $\mathrm{N}$ IV/C IV & $\mathrm{N}$ IV/O III \\
\hline \multicolumn{4}{|c|}{ Line fluxes used } \\
\hline 34 days & $13.5 \pm 2.0 / 2.99 \pm 1.2$ & $\ldots$ & $\ldots$ \\
\hline 44 days & $11.5 \pm 2.0 / 2.96 \pm 1.2$ & $\begin{array}{l}5.47 \pm 0.18 / 1.62 \pm 0.28^{\mathrm{a}} \\
5.47 \pm 0.18 / 2.48 \pm 0.22^{\mathrm{b}}\end{array}$ & $5.47 \pm 0.18 / 1.76 \pm 0.05$ \\
\hline 107 days & $5.57 \pm 0.8 / 2.24 \pm 1.0$ & $\begin{array}{l}5.17 \pm 0.15 / 0.85 \pm 0.13^{\mathrm{a}} \\
5.17 \pm 0.15 / 1.39 \pm 0.11^{\mathrm{b}}\end{array}$ & $5.17 \pm 0.15 / 1.25 \pm 0.05$ \\
\hline \multicolumn{4}{|c|}{ Observed line ratios } \\
\hline 34 days & $4.51 \pm 1.93$ & $\ldots$ & $\ldots$ \\
\hline 44 days & $3.89 \pm 1.71$ & $\begin{array}{l}3.37 \pm 0.59^{\mathrm{a}} \\
2.21 \pm 0.21^{\mathrm{b}}\end{array}$ & $3.11 \pm 0.14$ \\
\hline 107 days & $2.49 \pm 1.16$ & $\begin{array}{l}6.08 \pm 0.95^{\mathrm{a}} \\
3.71 \pm 0.31^{\mathrm{b}}\end{array}$ & $4.14 \pm 0.20$ \\
\hline 573 days & $2.49 \pm 1.16$ & $\begin{array}{l}6.08 \pm 0.95^{\mathrm{a}} \\
3.71 \pm 0.31^{\mathrm{b}}\end{array}$ & $4.14 \pm 0.20$ \\
\hline 621 days & $\cdots$ & $\begin{array}{l}6.08 \pm 0.95^{\mathrm{a}} \\
3.71 \pm 0.31^{\mathrm{b}}\end{array}$ & $4.14 \pm 0.20$ \\
\hline \multicolumn{4}{|c|}{ Derived ionic ratios $^{\mathrm{c}}$} \\
\hline Nov 11 & $37.9 \pm 16.2-30.3 \pm 13.0$ & $\ldots$ & $\ldots$ \\
\hline Nov 22 & $32.7 \pm 14.4-26.1 \pm 11.5$ & $\begin{array}{l}22.2 \pm 3.9-22.9 \pm 4.0^{\mathrm{a}} \\
14.6 \pm 1.4-15.0 \pm 1.4^{\mathrm{b}}\end{array}$ & $0.74 \pm 0.04-0.70 \pm 0.04$ \\
\hline Jan 23 & $20.9 \pm 9.8-16.7 \pm 7.8$ & $\begin{array}{l}40.1 \pm 6.3-41.3 \pm 6.5^{\mathrm{a}} \\
24.5 \pm 1.0-25.9 \pm 1.2^{\mathrm{b}}\end{array}$ & $0.99 \pm 0.05-0.93 \pm 0.05$ \\
\hline
\end{tabular}

Notes.

${ }^{\text {a }}$ Corrected for scattering.

b No correction for scattering.

${ }^{c}$ Assuming $n_{\mathrm{e}}$ in the range $10^{6}-10^{9} \mathrm{~cm}^{-3}$ and $T_{\mathrm{e}}=2 \times 10^{4} \mathrm{~K}$ and $E_{B-V}=0.027 \mathrm{mag}$.

the lower S/N and low spectral resolution of the STIS spectra (Figure 10). An additional complication is that the line sits on top of a broad electron-scattering feature centered on the $\mathrm{Si}$ III] $\lambda 1892.0$ line. This is also the case for the $\mathrm{N}$ III] $\lambda \lambda 1746.8-1754.0$ lines, but in this case it is easy to determine the background flux from the high-resolution COS spectra. An estimate of the systematic error in the N III] flux from STIS can consequently be obtained if one compares the COS and STIS fluxes for the same epoch. For the day 44 observation we get for the total N III] flux $(11.5 \pm 2.0) \times 10^{-14} \mathrm{erg} \mathrm{cm}^{-2} \mathrm{~s}^{-1}$ in the STIS observation, depending on where the "continuum" level is set, compared to $7.9 \times 10^{-14} \mathrm{erg} \mathrm{cm}^{-2} \mathrm{~s}^{-1}$ for the COS spectrum. For the day 107 spectrum, the corresponding numbers are $(5.57 \pm$ $0.8) \times 10^{-14} \mathrm{erg} \mathrm{cm}^{-2} \mathrm{~s}^{-1}$ and $(5.33 \pm 0.1) \times 10^{-14} \mathrm{erg} \mathrm{cm}^{-2} \mathrm{~s}^{-1}$, respectively. There is a tendency to overestimate the flux from the STIS observations, mainly from the difficulty in determining the continuum level. The same is likely to be the case for the C III] line.

As a consequence of this discussion, to minimize the systematic error we use the $\mathrm{N}$ III]/C III] ratio from the STIS observations, in spite of the lower $\mathrm{S} / \mathrm{N}$ in the STIS observations for $\mathrm{N}$ III] compared to the COS flux. To estimate the systematic error in the $\mathrm{C}$ III] flux, we have calculated the line flux for a range of assumptions about the background "continuum."The error may still be up to $\sim 50 \%$ and dominates the error budget in the relative $\mathrm{N}$ III/C III ratio. For the other line ratios, $\mathrm{N}$ IV]/C IV and $\mathrm{N}$ IV]/O III], we use fluxes from the COS spectra. For the $\mathrm{C}$ IV resonance doublet, we assume two limiting cases. In the first case we subtract the full absorption component from the emission component to correct for the scattering, as discussed in Section 3.4.2. This may underestimate the net emission by up to $\sim 60 \%$. In the other case, we neglect this correction and use the observed flux in the emission component. This is likely to overestimate the net emission. These limits should bracket the thermal excitation emission and is the best we can do without knowledge about the radius of the emission region. The adopted line ratios with errors are given in Table 8 . There is a fairly large scatter in both the $\left.\left.\mathrm{N}_{\mathrm{III}}\right] / \mathrm{C} \mathrm{III}\right]$ ratio and the $\left.\mathrm{N}_{\mathrm{IV}}\right] / \mathrm{C}_{\mathrm{IV}}$ ratio, reflecting the difficulties discussed above.

Using these line ratios, we can now derive relative ionic abundances. For the collision strengths and radiative transition rates, we use data from the Chianti database (Landi et al. 2012). In Table 8 we show the derived ionic abundances for the different dates and $T_{\mathrm{e}}=2 \times 10^{4} \mathrm{~K}$, typical of an X-ray-heated plasma. As discussed above, the results are not very sensitive to the temperature. To show the dependence on the assumed density we give the abundance ratios for both $n_{\mathrm{e}}=10^{6} \mathrm{~cm}^{-3}$ and $n_{\mathrm{e}}=10^{9} \mathrm{~cm}^{-3}$.

There are several things to note here. The scatter and large errors in the STIS fluxes are reflected in the $\mathrm{N}$ III/C III ratio. Taking an average of the three epochs, we get $n(\mathrm{~N}$ III $) / n(\mathrm{C}$ III $)=$ $30.5 \pm 13.7$ for $10^{6} \mathrm{~cm}^{-3}$ and $n(\mathrm{~N}$ III $) / n(\mathrm{C}$ III $)=24.3 \pm 11.0$ for $10^{9} \mathrm{~cm}^{-3}$. Also, the $\mathrm{N}$ IV/C IV ratio varies greatly with both epoch and the assumption about the scattering contribution. The scattering introduces an uncertainty of $\sim 60 \%$, and the variation between the different epochs amounts to 70\%-80\%. These variations are probably related to each other. A decrease in the continuum flux means that the scattering contribution will decrease. There may therefore be an evolution from a line dominated by scattering to a line where scattering is less important. The NIV/O III ratio does not have any of these complications because this ratio involves two intercombination lines. Consequently, it is relatively constant between the two epochs and also has small individual errors.

In addition to the observational errors and the uncertainty of the scattering correction, there is some uncertainty in the correspondence of the ionization zones. Although both the $\mathrm{C}$ III and $\mathrm{N}$ III zones, as well as the $\mathrm{CIV}, \mathrm{N}$ IV, and O III zones, 
nearly coincide in the X-ray photoionized models by Kallman \& McCray (1982), this is not obviously so for our case with a different ionizing spectrum and density, although it is likely that the narrow lines are indeed excited by X-rays (Section 4.1).

In spite of these caveats, the need for a very high $\mathrm{N} / \mathrm{C}$ ratio is clear. From the $\mathrm{N}$ III/C III and N IV/C IV ratios we find a "best value" of $n(\mathrm{~N}) / n(\mathrm{C})=25 \pm 15$. The $\mathrm{N} / \mathrm{O}$ ratio is better constrained to be $n(\mathrm{~N}) / n(\mathrm{O})=0.85 \pm 0.15$. For comparison, the corresponding solar ratios are $n(\mathrm{~N}) / n(\mathrm{C})=0.25$ and $n(\mathrm{~N}) / n(\mathrm{O})=0.14$ (Asplund et al. 2009).

\section{DISCUSSION}

\subsection{The Narrow Circumstellar Lines}

Our analysis in Section 3 provides several pieces of important information about the SN and its CSM that provide clues to the nature of the progenitor and its evolutionary status. In particular, we have evidence for a CSM of the progenitor with a velocity of $\sim 100 \mathrm{~km} \mathrm{~s}^{-1}$ and a density of $3 \times 10^{6}-10^{8} \mathrm{~cm}^{-3}$. Further, the derived $\mathrm{CNO}$ abundances indicate a large nitrogen enrichment, $n(\mathrm{~N}) / n(\mathrm{C})=25 \pm 15$ and $n(\mathrm{~N}) / n(\mathrm{O})=0.85 \pm 0.15$, typical of $\mathrm{CNO}$ processed gas.

A large $\mathrm{CNO}$ enrichment has been observed for a number of $\mathrm{SNe}$, either in the ejecta itself or in the CSM. This includes SN 1979C (IIL), SN 1995N (IIn), SN 1998 S (IIn), SN 1993J (IIb), and SN 1987A (IIpec) (see Fransson et al. 2005 for a summary of these with references). As for potential progenitors, CNO enrichment is observed for several types of evolved massive stars. In particular, this is consistent with an LBV scenario for the progenitor. The best-studied cases are for Eta Carinae and AG Carinae, which we discuss below.

Hillier et al. (2001) make a detailed analysis of STIS observations of the central source in Eta Carinae. The mass-loss rate is determined to be $\sim 10^{-3} \quad M_{\odot} \mathrm{yr}^{-1}$ for a wind velocity of $\sim 500 \mathrm{~km} \mathrm{~s}^{-1}$. From the weakness of the electron scattering wings, they conclude that the wind has to be clumpy, with a filling factor of $\sim 10 \%$. Hillier et al. also find that the $\mathrm{N}$ abundance in the primary star is at least a factor of 10 higher than solar, while $\mathrm{C}$ and $\mathrm{O}$ are severely depleted. In the nebula of Eta Carinae, the CNO abundances can be determined more reliably. In particular, Dufour et al. (1997) and Verner et al. (2005) find that $\mathrm{N}$ is enhanced by a factor of $10-20$, and $\mathrm{C}$ and $\mathrm{O}$ are depleted by a factor of 50-100. Smith \& Morse (2004) also find a strong radial gradient in the $\mathrm{N} / \mathrm{O}$ ratio in Eta Carinae, with a high $\mathrm{N} / \mathrm{O}$ ratio close to the star, decreasing outward.

Most of the mass in the CSM of Eta Carinae is in a molecular shell surrounding the central star. Smith (2006) estimates a total mass of $\sim 11 M_{\odot}$. The distance from the star to this hourglass-shaped shell is between $3 \times 10^{16} \mathrm{~cm}$ and $3 \times 10^{17} \mathrm{~cm}$. Especially interesting is that Smith (2006) derives a density of $\gtrsim 3 \times 10^{6} \mathrm{~cm}^{-3}$ for the molecular shell around Eta Carinae. This density bound is similar to what we find for the CSM of SN 2010jl. In addition, gas with similar densities are seen even closer, at distances $\sim 10^{15} \mathrm{~cm}$ from the star, as [Fe II] emission.

Besides Eta Carinae, AG Carinae is one of the most extensively studied LBV stars, showing a variation of the S Doradus type. Over the two periods studied, the mass-loss rate varied in the range $(1.5-3.7) \times 10^{-5} M_{\odot} \mathrm{yr}^{-1}$, and the wind velocity varied in the range $300-105 \mathrm{~km} \mathrm{~s}^{-1}$, in antiphase with the mass loss (Groh et al. 2009). It has clearly undergone a more intense mass loss period earlier, as is apparent from the fact that it has a circumstellar nebula with an ionized mass of $\sim 4.2 M_{\odot}$ and a dy- namical age of $\sim 8.5 \times 10^{3} \mathrm{yr}$ (Nota et al. 1995). An even higher mass of $\sim 25 M_{\odot}$ in the neutral medium has been estimated based on IR imaging, assuming a standard dust-to-gas ratio of 1:100 (Voors et al. 2000). From a spectral analysis of the central star, Groh et al. (2009) find a $\mathrm{N}$ mass fraction of $11.5 \pm 3.4$ times solar, while $\mathrm{C}$ is only $0.11 \pm 0.03$ times solar, and $\mathrm{O}$ is $0.04 \pm 0.2$ times solar, clearly indicating $\mathrm{CNO}$ processed material. The abundances in the nebula are more uncertain, with an $\mathrm{N} / \mathrm{O}$ ratio of $6 \pm 2$, compared to $39_{-18}^{+28}$ for the star. It is likely that the surface of the star has undergone more $\mathrm{CNO}$ processing than the nebula.

The wind velocity we find for SN 2010j1, $\sim 100 \mathrm{~km} \mathrm{~s}^{-1}$, is not that of a red supergiant, whose velocities are in the range $10-40 \mathrm{~km} \mathrm{~s}^{-1}$ (Jura \& Kleinmann 1990), so we rule these out as progenitors. Here we differ from Zhang et al. (2012), who find a wind velocity of $28 \mathrm{~km} \mathrm{~s}^{-1}$, but this is a result of their use of the velocity of maximum absorption in the P-Cygni profile and not the maximum blue velocity. The maximum absorption velocity we observe is also considerably higher than theirs (Figure 17). Their conclusion that the progenitor was a red supergiant is based on a wind velocity that is too low. We find a velocity that is more typical of LBV stars, which have velocities in the range 100-1000 $\mathrm{km} \mathrm{s}^{-1}$ (Smith et al. 2011b). The velocity we find, $\sim 100 \mathrm{~km} \mathrm{~s}^{-1}$, is on the low end of this range, which may be a potential problem with the LBV interpretation. However, the expansion velocity of the molecular shell in Eta Carinae is highly anisotropic, ranging from $\sim 60 \mathrm{~km} \mathrm{~s}^{-1}$ at the equator to $\sim 650 \mathrm{~km} \mathrm{~s}^{-1}$ at the poles (Smith 2006), and is likely to vary substantially with time. As we noted above, the velocity in the high mass loss phase of AG Carinae is very similar to the one we derive for SN 2010j1.

Although it is clear that the narrow lines originate in fairly dense circumstellar gas, it is not obvious what excites them. The recombination time is $\sim 1 / \alpha n_{\mathrm{e}} \sim 5.8\left(n_{\mathrm{e}} / 10^{7} \mathrm{~cm}^{-3}\right)^{-1}$ days for $\mathrm{H}$ and $T_{\mathrm{e}} \sim 10^{4} \mathrm{~K}$. The gas is therefore in near equilibrium with the ionizing radiation. An early, undetected UV burst is therefore not likely to be important for the excitation. The widths of the lines, $\lesssim 100 \mathrm{~km} \mathrm{~s}^{-1}$, also make it unlikely that the gas is shock ionized.

Instead, we believe that the lines are excited by the same $\mathrm{X}$-rays as are observed with the Chandra satellite (Chandra et al. 2012b). As we discuss in Section 4.3, these are likely to come from fast shocks connected to an anisotropic, high-velocity ejection. We can estimate the state of ionization from the models in Kallman \& McCray (1982). With an X-ray luminosity of $\sim 3 \times 10^{41} \mathrm{erg} \mathrm{s}^{-1}$, corresponding to the absorbed flux of $\sim 10^{-12}$ erg $\mathrm{cm}^{-2} \mathrm{~s}^{-1}$ (Chandra et al. $2012 \mathrm{~b}$ ), the ionization parameter, $\zeta=L / n_{\mathrm{e}} r^{2}$, becomes $\zeta \sim 300\left(n_{\mathrm{e}} / 10^{7} \mathrm{~cm}^{-3}\right)^{-1}\left(r / 10^{16} \mathrm{~cm}\right)^{-2}$. From the optically thin Model 1 in Kallman \& McCray (1982), we find that the presence of $\mathrm{N}$ III-N v requires $\log \zeta \sim 0-1$. Optically thick models with a density of $10^{11} \mathrm{~cm}^{-3}$ have the same ions at a somewhat higher ionization parameter, $\log \zeta \sim 1.5$. The presence of [Fe XIV] (Section 3.4.3) is also consistent with this ionization parameter. The observed X-ray luminosity indicates a distance of $\sim(2-20) \times 10^{16}\left(n_{\mathrm{e}} / 10^{7} \mathrm{~cm}^{-3}\right)^{-1 / 2} \mathrm{~cm}$. The radius of the shock at 500 days after outburst is $\sim 1.3 \times$ $10^{16}\left(V_{\mathrm{s}} / 3000 \mathrm{~km} \mathrm{~s}^{-1}\right)(t / 500$ days $) \mathrm{cm}$, where $V_{\mathrm{s}}$ is the shock velocity. There is therefore a rough consistency between the estimated distance of the narrow line emitting region and the location of the SN shock wave.

This estimate of the ionization parameter is approximate for several reasons. First, the density has a considerable uncertainty. 
Further, as we discuss below, the shock velocity, and therefore the shock radius, is also uncertain and may be in the range 500-3000 $\mathrm{km} \mathrm{s}^{-1}$. Finally, the X-ray models in Kallman \& McCray (1982) are not completely appropriate because these assume a $10 \mathrm{keV}$ bremsstrahlung spectrum, and the X-ray spectrum of SN 2010jl is heavily absorbed, having a deficit of photons below $\sim 1 \mathrm{keV}$. The Kallman \& McCray (1982) models were also calculated for a density of $10^{11} \mathrm{~cm}^{-3}$, while the narrow lines in SN 2010jl indicate a considerably lower density. Nevertheless, this estimate shows that it is likely that the narrow lines originate in X-ray ionized gas at a distance and density comparable to the molecular shell of Eta Carinae discussed above. In Section 4.7 we discuss more detailed photoionization calculations that confirm these estimates.

The fact that we observe fairly "normal" P-Cygni profiles with both a deep absorption and a red emission wing implies that the CSM at the distance where the narrow lines arise must be fairly symmetrically distributed, unlike the distribution for the broad component as described below. This is discussed further in Section 4.7.

\subsection{Origin of the Dust Emission}

The NIR excess seen in the photometry in Figures 2 and 4, as well as the X-shooter spectrum in Figure 20, is most likely a result of hot dust. From the photometric light curve in Figure 3 we see that by day 400 the flux in the NIR is higher than that in the optical. This NIR/optical ratio increases with time, and at the last epochs the NIR luminosity dominates completely.

In Section 3.2 we used simple blackbody fits of the optical and NIR photometry. We have investigated this in more detail following Kotak et al. (2009). Here we have used the grain emissivities from Draine \& Lee (1984) and Laor \& Draine (1993) and the escape probability formalism from Osterbrock \& Ferland (2006). For the grain sizes we use the MRN distribution with $d n / d a \propto a^{-3.5}$ (Mathis et al. 1977) with a minimum grain size $0.005 \mu \mathrm{m}$ and a maximum size $0.05 \mu \mathrm{m}$. We then fit the sum of the dust spectrum with a given optical depth in the $V$ band, $\tau_{\mathrm{V}}$, and a photospheric blackbody spectrum, minimizing the chisquare with respect to the photospheric effective temperature and radius $T_{\text {eff }}$ and $R_{\text {phot }}$ and the corresponding parameters for the dust shell, $T_{\text {dust }}, R_{\text {dust }}$. A more detailed modeling, calculating the temperature of the dust as a function of radius, as in Andrews et al. (2011), requires assumptions about the geometry and is outside the scope of this paper.

In Figure 27 we show an example of this kind of fit for day 465 for which we have observations at $3.6 \mu \mathrm{m}$ and $4.5 \mu \mathrm{m}$.

From this figure it is clear that without any observations longward of $4.5 \mu \mathrm{m}$ it is impossible to discriminate between the different compositions and only marginally between different optical depths for the dust. This is in agreement with the modeling by Andrews et al. (2011).

The main difference between the optically thin and thick models is the larger radius of the former in order to produce the required luminosity. The dust temperature is lower in the optically thin models. At 465 days the best-fit silicate model in Figure 27 with an optical depth in the $V$ band of $\tau_{\mathrm{V}}=0.04$ had $T_{\text {dust }}=1680 \mathrm{~K}$ and $R_{\text {dust }}=3.3 \times 10^{17} \mathrm{~cm}$. The photospheric values were less affected with $T_{\text {eff }}=8900 \mathrm{~K}$ and $R_{\text {phot }}=6.0 \times 10^{14} \mathrm{~cm}$. The corresponding graphite model had $T_{\text {dust }}=1360 \mathrm{~K}, R_{\text {dust }}=7.8 \times 10^{17} \mathrm{~cm}, T_{\text {eff }}=8600 \mathrm{~K}$, and $R_{\text {phot }}=6.6 \times 10^{14} \mathrm{~cm}$. These values should be compared to $T_{\text {dust }}=2040 \mathrm{~K}, R_{\text {dust }}=2.2 \times 10^{16} \mathrm{~cm}, T_{\text {eff }}=9200 \mathrm{~K}$, and

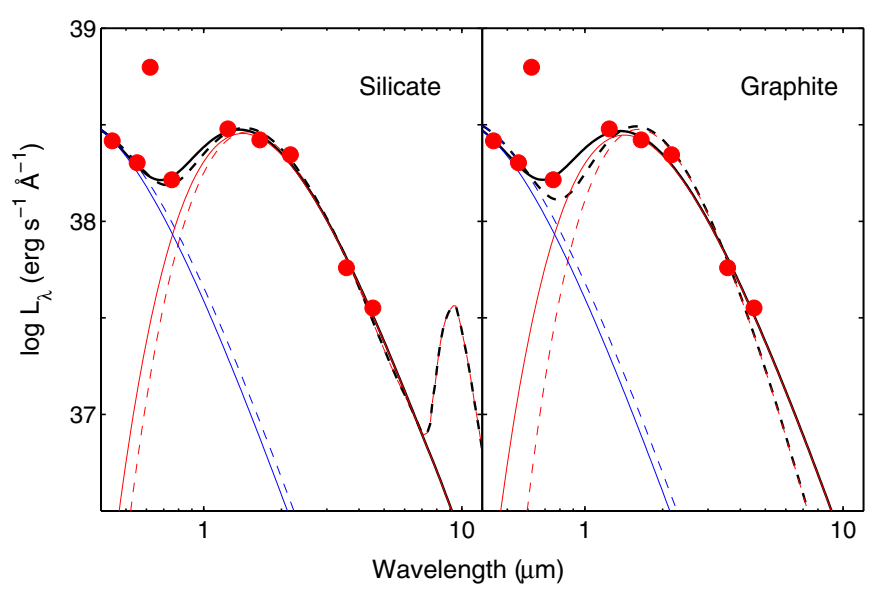

Figure 27. SED fits to the extinction-corrected photometry at 465 days for two different dust compositions, silicates, and graphite. The blue curves show the photospheric contribution, the red show the dust component, and the black curves the total. The dashed lines show the optically thin models with $\tau_{\mathrm{V}}=0.04$, and the solid lines show the corresponding optically thick models. As discussed in the text, the $R$ band, which is dominated by the $\mathrm{H} \alpha$ line, is not included in the fits. We note the $10 \mu \mathrm{m}$ silicate feature for the optically thin model, which is the main discriminator for the dust composition.

(A color version of this figure is available in the online journal.)

$R_{\text {phot }}=5.6 \times 10^{14} \mathrm{~cm}$ for the optically thick model (Table 5 ). We find similar results for the other epochs. As Figure 27 shows, the separation between the dust and photospheric components are only marginally affected. The light curves of the photospheric and dust components based on the blackbody fits in Section 3.2 should therefore be accurate.

As an independent check we find from a fit to the NIR continuum of the X-shooter spectrum at 461 days in Figure 20 a dust temperature $\sim 1870 \mathrm{~K}$ and radius $R_{\text {dust }} \sim 2.5 \times 10^{16} \mathrm{~cm}$ for the optically thick model, in good agreement with that derived from the broadband photometry. For the dates with only broadband photometry, we give $R_{\text {dust }}$ in Figure 5 .

It should be noted that the blackbody radius only represents a lower limit to the NIR-emitting region. A covering factor, $f$, less than unity will increase the radius by $R_{\text {dust }} \propto f^{-1 / 2}$. As discussed above, an optically thin model would increase this further.

The blackbody radius can be compared to the shock radius, given by $R_{\mathrm{s}} \sim V_{\mathrm{s}} t \sim 1.0 \times 10^{16}\left(V_{\mathrm{s}} / 3000 \mathrm{~km} \mathrm{~s}^{-1}\right)(\mathrm{t} / 400$ days $) \mathrm{cm}$, where we have scaled the velocity to what we believe is the maximum velocity of the shock (Section 4.3). With this velocity, or higher, the blackbody radius is comparable to the shock radius. In addition, a lower shock velocity is likely in the directions where the column density is larger than that inferred from the X-rays, which would give a smaller shock radius (Section 4.7).

The blackbody and shock radii can also be compared to the evaporation radius, given by $R_{\text {evap }}=\left[L_{\max } Q_{\text {abs }} /\right.$ $\left.\left(16 \pi \sigma T_{\text {evap }}^{4} Q_{\text {emiss }}\left(T_{\text {evap }}\right)\right)\right]^{1 / 2}$ (Draine \& Salpeter 1979). Here $L_{\max }$ is the maximum bolometric luminosity of the $\mathrm{SN}, Q_{\mathrm{abs}}$ is the wavelength-averaged dust absorption efficiency over the $\mathrm{SN}$ spectrum, $Q_{\mathrm{emiss}}$ is the Planck-averaged dust emission efficiency, $\sigma$ is the Stefan-Boltzmann constant, and $T_{\text {evap }}$ is the evaporation temperature; $Q_{\mathrm{abs}} \propto a$, where $a$ is the size of the dust grain. The parameter $Q_{\text {abs }} / Q_{\text {emiss }}$ depends upon the evaporation temperature of the dust, the grain size of the dust, and the effective temperature of the $\mathrm{SN}, T_{\text {eff }}$. For our calculations of $R_{\text {evap }}$ we adopted values of $T_{\text {evap }}=1900 \mathrm{~K}$ for graphite and 
Table 9

Dust Evaporation Radii for $L_{\mathrm{bol}}=10^{43} \mathrm{erg} \mathrm{s}^{-1}$

\begin{tabular}{|c|c|c|c|}
\hline $\begin{array}{l}a \\
(\mu \mathrm{m})\end{array}$ & $\begin{array}{l}T_{\text {eff }} \\
(\mathrm{K})\end{array}$ & $\begin{array}{c}R_{0} \text { evap } \\
\left(10^{17} \mathrm{~cm}\right) \\
\text { Silicates }^{\mathrm{a}}\end{array}$ & $\begin{array}{c}R_{0 \text { evap }} \\
\left(10^{17} \mathrm{~cm}\right) \\
\text { Graphite }^{\mathrm{a}}\end{array}$ \\
\hline 0.001 & 10,000 & 2.59 & 1.43 \\
\hline 1.0 & & 1.30 & 0.34 \\
\hline 0.001 & 6,000 & 0.86 & 0.86 \\
\hline 1.0 & & 0.72 & 0.35 \\
\hline
\end{tabular}

Note. ${ }^{\text {a }}$ Assuming $T_{\text {evap }}=1500 \mathrm{~K}$ for silicates and $T_{\text {evap }}=$ $1900 \mathrm{~K}$ for graphite.

$T_{\text {evap }}=1500 \mathrm{~K}$ for silicates and assumed $T_{\mathrm{SN}}=6000 \mathrm{~K}$ and $10,000 \mathrm{~K}$. For both graphite and for silicates we use grain emissivities from Draine \& Lee (1984) and Laor \& Draine (1993). Because $R_{\text {evap }} \propto L_{\max }^{1 / 2}$, we give values for $L_{\max }=10^{43} \mathrm{erg} \mathrm{s}^{-1}$ in Table 9, which can then be scaled to different luminosities.

We note that $R_{\text {evap }}$ is not very sensitive to $T_{\text {eff }}$, except for very small dust grains. As a typical value at shock breakout we use $T_{\text {eff }}=10^{4} \mathrm{~K}$ for our estimates below. The peak luminosity of the $\mathrm{SN}$ was $L_{\max } \sim 3 \times 10^{43} \mathrm{erg} \mathrm{s}^{-1}$ (Figure 3). Using a grain size of $\sim 0.001 \mu \mathrm{m}$, we get $R_{\text {evap }} \sim 2.5 \times 10^{17} \mathrm{~cm}$ for graphite and $R_{\text {evap }} \sim 4.5 \times 10^{17} \mathrm{~cm}$ for silicates. For a grain size of $\sim 1 \mu \mathrm{m}$, the corresponding values are $R_{\text {evap }} \sim 6.0 \times 10^{16} \mathrm{~cm}$ and $R_{\text {evap }} \sim 2.3 \times 10^{17} \mathrm{~cm}$, respectively.

The dust temperatures we find at $\lesssim 500$ days, $1600-2000 \mathrm{~K}$, are close to the evaporation (or formation) temperature for dust. It may therefore either be newly formed dust, close to the shock, or dust heated too close to the evaporation temperature by either the radiation from the $\mathrm{SN}$ or the shock wave. We now discuss different scenarios for the origin of the dust emission.

The cool, dense shell formed behind a radiative shock has often been mentioned as a favorable place for dust formation. The formation and survival of dust in this environment is, however, difficult to explain at epochs earlier than $\sim 320$ days. Dust formation in the dense postshock gas requires temperatures of $\lesssim 1900 \mathrm{~K}$. At these epochs, the shock is most likely in the optically thick region of the $\mathrm{SN}$. The photospheric temperature at these epochs is $\gtrsim 7000 \mathrm{~K}$ (Table 5). Even if the shock is radiative, it is therefore unlikely to cool to less than this temperature and may be higher. A related argument comes from noticing that the shock radius is well within the evaporation radius, even at $\sim 200$ days. As Table 9 shows, $R_{\text {evap }} \gtrsim 3 \times 10^{16} \mathrm{~cm}$ for a luminosity of $\sim 10^{43} \mathrm{erg} \mathrm{s}^{-1}$ at 200 days, independent of dust size or composition for $T_{\text {eff }} \gtrsim 6000 \mathrm{~K}$. It is therefore difficult to see how any dust could form close to the shock region for a realistic shock velocity.

It is also difficult to see how the large IR luminosity at late epochs can be produced by such dust. The photospheric luminosity is then low, and the X-ray luminosity is likely to be less than that at the last observation by Chandra et al. (2012b) on day $373, \sim 7 \times 10^{41} \mathrm{erg} \mathrm{s}^{-1}$, which is a factor of $\sim 5$ lower than the NIR luminosity at 500 days. This agrees with the conclusions by Andrews et al. (2011), although with somewhat different arguments.

Reprocessing of radiation from the SN, possibly resulting in evaporation of preexisting dust or heating and evaporation of the dust by the shock, is more likely. As Andrews et al. conclude, there is evidence for preexisting dust around the progenitor star. The dust heating mechanism is, however, not clear. Previous treatments of dust in Type IIn SNe have discussed both heating by the radiation from the $\mathrm{SN}$ in combination with an echo and collisional heating by the hot gas behind the shock (e.g., Gerardy et al. 2002; Fox et al. 2010).

We first discuss the case where the dust emission comes from dust that is collisionally evaporated by the shock of the SN. As discussed in the next section, there is also direct evidence for such a velocity component in other Type IIn SNe. Massive dust shells into which the shock is propagating are also natural if the progenitor is an LBV. However, if we compare the shock radius with the evaporation radius at early epochs, we find that even the lowest values of the latter, $R_{\text {evap }} \sim 7 \times 10^{16} \mathrm{~cm}$, are considerably larger than the shock radius at the first epochs, $R_{\mathrm{s}} \sim 2.6 \times 10^{15}\left(V_{\mathrm{s}} / 3000 \mathrm{~km} \mathrm{~s}^{-1}\right)(\mathrm{t} / 100$ days $) \mathrm{cm}$, where we have scaled to the velocity derived from the temperature of the $\mathrm{X}$-rays. This radius should be seen as an upper limit to the shock radius. We therefore exclude this possibility.

A more attractive alternative is based on an echo from radiatively heated dust, as has been discussed for other Type IIns. The plateau in the IR light curve between $\sim 200$ and $\sim 450$ days and a slow decline thereafter (Figures 2 and 3 ) would then be expected. Also, the energetics may be consistent with this. The integrated optical luminosity from the SN during the first $\sim 400$ days was $\sim 3.2 \times 10^{50} \mathrm{erg}$. In addition, there is a considerable luminosity in the UV, as well as at shorter wavelengths. This can be compared to the integrated luminosity in the dust component, which we estimate from the blackbody fits in Figure 5; we find a total energy of $\sim 2.7 \times 10^{50} \mathrm{erg}$. There is therefore reasonable consistency between the energy emitted from the central source and that emitted by the dust. It does, however, require a large covering factor of the dust, as well as a high optical depth in these directions, which is consistent with the blackbody shape of the spectrum. As Andrews et al. discuss, this may indicate an anisotropic geometry; they discuss torus models with different inclinations.

To explain the plateau as an echo, the inner radius would have to be $\geq 450 / 2$ light days or $\sim 6 \times 10^{17} \mathrm{~cm}$, depending on the inclination of a disk or torus. At the same time the high temperature indicates that the inner radius is close to the evaporation radius. A radius of $\sim 6 \times 10^{17} \mathrm{~cm}$ is, however, considerably larger than the earlier estimates for $R_{\text {evap }}$, even if the luminosity used for this estimate may be an underestimate, considering the fact that only the observed wavelengths were included and also that the peak luminosity may have been before the first observations. A solution to this discrepancy may be that most of the emission comes from very small grains. Some support for the presence of small grains in winds comes from observations and modeling of the emission from some Orich asymptotic giant branch stars in the LMC, where a grain distribution with a minimum size of $0.01 \mu \mathrm{m}$ to $0.1 \mu \mathrm{m}$ gave the best fit (Sargent et al. 2010). Although the most appealing model, this scenario has some difficulties in simultaneously explaining both the light curve and the high dust temperature, unless one stretches the parameters.

We have calculated light curves from an echo from a spherically symmetric dust shell of inner radius $6 \times 10^{17} \mathrm{~cm}$. We, however, find that the rise of the light curve occurs too rapidly compared to the observed NIR light curve in Figure 3 and the total dust luminosity in Figure 5. Emmering \& Chevalier (1988) have studied echoes from asymmetric dust distributions. As shown in their Figures 2 and 3, more disk-like distributions give a considerably slower rise for low values of the inclination of the disk or torus. A low value of the inclination also agrees with the low reddening in the spectrum of the $\mathrm{SN}$, while a high optical 
depth in the disk is needed for the high dust to SN luminosity, discussed above. Even more asymmetric distributions with most of the dust behind the $\mathrm{SN}$ relative to our LOS may also be consistent with the light curve, although we have not studied this quantitatively. The increase of the dust-emitting area and simultaneous decrease of the dust temperature (Figure 5) are, however, qualitatively consistent with an echo, as the light echo paraboloid expands to larger radii into dust with decreasing temperature.

In the discussion about different dust emission mechanisms for Type IIn SNe Fox et al. (2011; see also Gerardy et al. 2002) proposed a scenario where preexisting dust was radiatively heated by the radiation from ongoing circumstellar interaction. Although the heating of the dust is radiative, echo effects are less important, and the shell can be at a smaller evaporation radius, without invoking small grains. If the late luminosity, including all wavelengths, is of the same order as the maximum bolometric luminosity, this may account for the high temperature observed. The main problem is that the luminosity of the late IR emission is high, $\gtrsim 5 \times 10^{42} \mathrm{erg} \mathrm{s}^{-1}$ at 500 days, while the optical flux from the $\mathrm{SN}$ is decreasing rapidly. This scenario would therefore require a very large EUV-X-ray luminosity. There may be some indication of this from the $\mathrm{H} \alpha$ luminosity, as discussed in Section 4.7.

\subsection{The Broad Emission Lines}

The profiles of the broad lines give important information about the structure of the envelope and the dynamics of the shock wave. As we discuss in the previous and next sections, dust formation has severe problems in both the ejecta and in connection to the shock at early epochs due to the high luminosity and temperature of the radiation. Instead, the blueshift of the lines is more easily explained as a result of the line formation and dynamics in the outer parts of the extensive envelope/CSM of the progenitor star, as was also suggested as a possible alternative by Smith et al. (2012b). As discussed in detail by, e.g., Chevalier \& Irwin (2011), for a radiationdominated shock in an extended envelope, the radiation starts to escape when $\tau_{\mathrm{e}} \lesssim c / V_{\mathrm{s}}$. The region outside the shock is therefore optically thick, and line radiation emitted here will be Compton scattered and can give rise to the broad wings observed, even if the emission is coming from regions that have not yet been accelerated by the shock radiation. Only when the region with $\tau_{\mathrm{e}} \lesssim 1$ becomes accelerated will the macroscopic velocity become dominant.

An emission line undergoing electron scattering in a hot medium at rest will give rise to a symmetric line profile because the velocity broadening is provided by the microscopic, thermal velocities of the electrons (Münch 1948). However, if the medium is moving with a bulk velocity toward us, this can introduce a blueshift of the line profile. For a spherically symmetric expansion one expects a redshift of the line peak (Auer \& van Blerkom 1972; Fransson \& Chevalier 1989). This is clearly not the case here. However, if the scattering medium is primarily moving coherently toward us, one may instead get a blueshift of the peak, although it is still symmetric around the expansion velocity. We believe that this is the case here.

We can test this interpretation quantitatively by first shifting each line in Figure 12 by a macroscopic velocity, $V_{\text {bulk }}$, to the red. We adjust this so that a reflection about zero velocity gives a best $\chi^{2}$ fit for Doppler shifts in the range $\sim 1000-5000 \mathrm{~km} \mathrm{~s}^{-1}$, $\left.\chi^{2}=\sum_{i}\left[F_{\lambda}\left(V-V_{\text {bulk }}\right)-F_{\lambda}\left(-\left(V-V_{\text {bulk }}\right)\right)\right]^{2}\right] / \sigma^{2}$, where $V$ is the velocity rest wavelength and $\sigma$ is the rms of the flux.
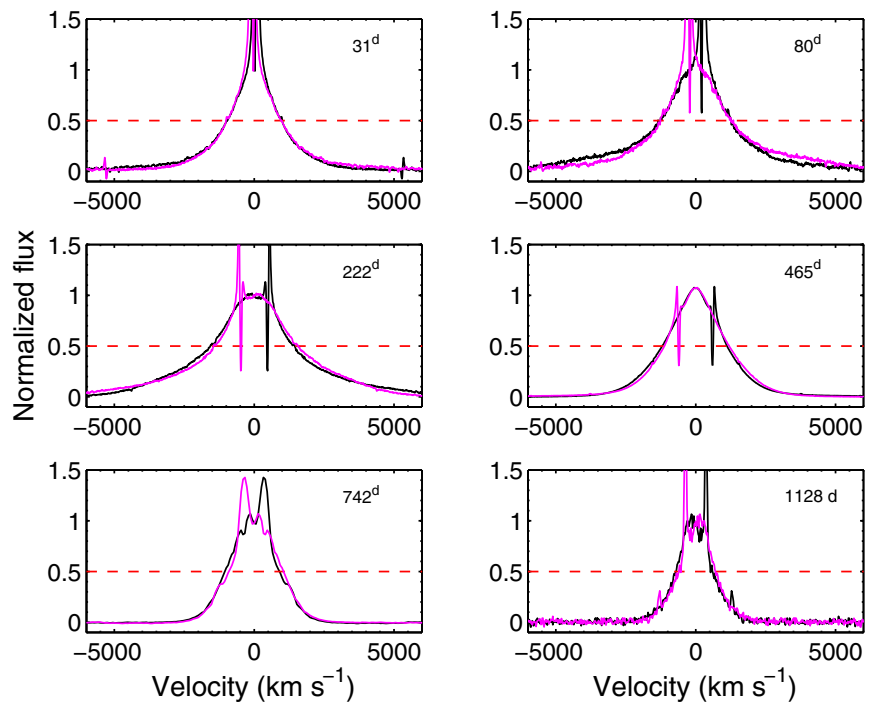

Figure 28. Broad $\mathrm{H} \alpha$ line for selected dates after shifting the line to the red by a velocity given in the upper panel of Figure 29 (black line). The magenta line gives the reflected line profile. Note the nearly perfect symmetry between the red and blue wings, characteristic of electron scattering. The hornsclose to the center of the line are due to the P-Cygni absorption from the narrow component coming from the CSM, which is close to the rest velocity of the host galaxy. The separation between the hornsis therefore a measure of the velocity shift of the broad line component.

(A color version of this figure is available in the online journal.)

The lower velocity limit is chosen to avoid influence on the line profile by the narrow P-Cygni absorption and emission and the scattering wings of this emission. We treat $V_{\text {bulk }}$ as a free parameter representing the macroscopic velocity of the emitting gas. The result of this procedure for a selection of days is shown in Figure 28.

From this figure we see that this simple, linear transformation results in nearly perfectly symmetric line profiles between the red and blue wings for essentially all epochs. This therefore argues that the blueshift in Figure 12 is due to a macroscopic velocity. In addition, the symmetric line profiles are a natural result of electron scattering. Other processes, like dust or other absorption processes, would result in asymmetric lines about the peak in flux, with a time-dependent line shape (e.g., Lucy et al. 1989). Electron scattering in an expanding medium that is optically thick, however, naturally results in symmetric profiles, as demonstrated explicitly below.

In the upper panel of Figure 29 we show with blue markers the $V_{\text {bulk }}$ as a function of time. In this panel we also show the velocity of the peak of the broad component as red markers. For the early epochs this agrees well with $V_{\text {bulk, but it is }}$ somewhat higher for the later epochs. The broad agreement of these two determinations is another way of showing the symmetry of the line around the velocity-shifted peak of the broad component. Because the peak velocity is more difficult to determine in the late spectra and is somewhat influenced by the narrow component, we prefer to use $V_{\text {bulk }}$ as the macroscopic velocity for the rest of the paper. To quantify the width of the line profiles we use the FWHM as measured from the continuumsubtracted line profiles. This is shown in the lower panel of Figure 29.

During the first 300-400 days there is an increase in $V_{\text {bulk }}$ to $\sim 700 \mathrm{~km} \mathrm{~s}^{-1}$. After this epoch the velocity decreases slightly and then becomes nearly constant at $\sim 400-500 \mathrm{~km} \mathrm{~s}^{-1}$. Considering the errors in the last observations, the significance of this 

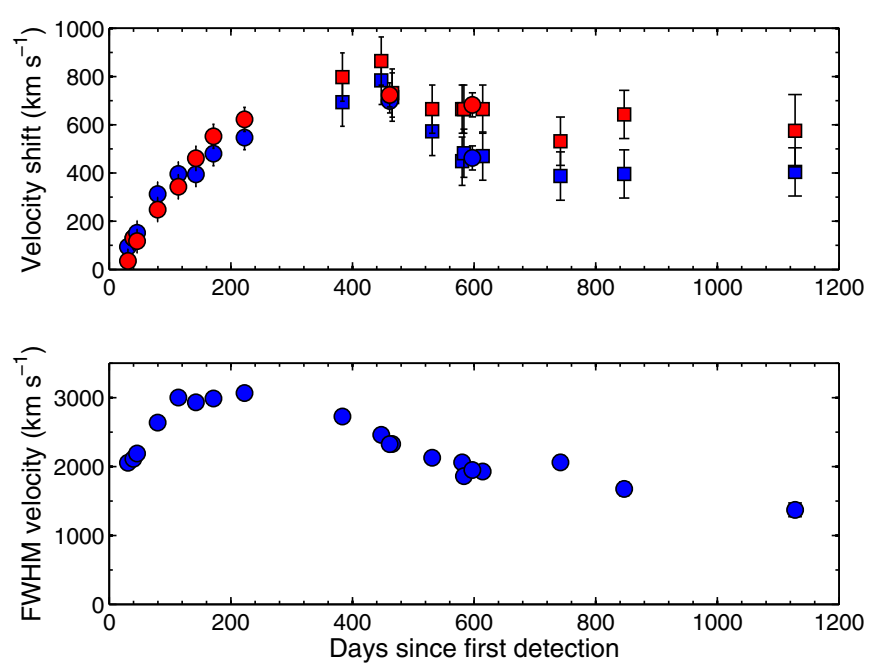

Figure 29. Upper panel: velocity shift, representing a macroscopic velocity of the $\mathrm{H} \alpha$-emitting region as a function of time. Blue markers represent $V_{\text {bulk }}$ from measurements of the symmetry of the lines, as discussed in the text, and red markers represent the peak velocity of the broad component. Circles represent measurements with medium-resolution spectra, and the squares are measurements from low-resolution spectra. Lower panel: FWHM of $\mathrm{H} \alpha$ as a function of time.

(A color version of this figure is available in the online journal.)

decrease is marginal. The FWHM increases from $\sim 2000 \mathrm{~km} \mathrm{~s}^{-1}$ to $\sim 3000 \mathrm{~km} \mathrm{~s}^{-1}$ during the first $\sim 200$ days and then decreases slowly to $\sim 1500 \mathrm{~km} \mathrm{~s}^{-1}$ on day 1128 .

Before discussing this result in more detail, we first test the electron scattering interpretation further by fitting the line profiles for a few different dates. This is done by a Monte Carlo code developed for this purpose, which is similar to that developed by Auer \& van Blerkom (1972) and Chugai (2001). The details of this are discussed at length in, e.g., Pozdniakov et al. (1977) and Gorecki \& Wilczewski (1984). Except for the Compton recoil, which is negligible at these wavelengths, this calculates the line profile for an arbitrary temperature and spatial distribution of electrons. In this calculation, relativistic effects are unimportant and are ignored. Because the macroscopic bulk velocity can be transformed away in the way described above, we only consider scattering in a static medium.

For photons injected at a specific optical depth, $\tau_{\mathrm{e}}$, the parameters determining the line shape are only $\tau_{\mathrm{e}}$ and temperature $T_{\mathrm{e}}$. The optical depth determines the average number of scatterings, $N_{\mathrm{sc}} \approx \tau_{\mathrm{e}}^{2}$. The thermal velocity of the electrons is $v_{\text {rms }}=674\left(T_{\mathrm{e}} / 10^{4} \mathrm{~K}\right)^{1 / 2} \mathrm{~km} \mathrm{~s}^{-1}$. The FWHM of the line is $\sim N_{\mathrm{sc}}^{1 / 2} v_{\mathrm{rms}} \approx 674 \tau_{\mathrm{e}}\left(T_{\mathrm{e}} / 10^{4} \mathrm{~K}\right)^{1 / 2} \mathrm{~km} \mathrm{~s}^{-1}$. For a constant electron temperature medium with no internal emission, our Monte Carlo calculations show that the FWHM can be fitted more accurately with $\Delta v_{\text {FWHM }}=900\left(T_{\mathrm{e}} / 10^{4} \mathrm{~K}\right)^{1 / 2} \tau_{\mathrm{e}} \mathrm{km} \mathrm{s}^{-1}$. A similar scaling also applies if the photons are internally generated. This means that there is some degeneracy in temperature and optical depth for a given $\Delta v_{\text {HWHM }}$. There is, however, a constraint on $\tau_{\mathrm{e}}$ : the luminosity of the unscattered fraction of photons relative to those scattered is approximately $\exp \left(-\tau_{\mathrm{e}}\right)$.

In Figure 30 we show fits of the $\mathrm{H} \alpha$ profile for two different dates, day 31 and day 222, covering both the very early and late phases. In this model we used an $n_{\mathrm{e}} \propto r^{-2}$ density and assumed that the photons are injected in the scattering region with an emissivity $\propto n_{\mathrm{e}}^{2}$, as applies if recombination dominates the $\mathrm{H} \alpha$ emission. Note that the narrow component of the $\mathrm{H} \alpha$ line is at the rest velocity of the galaxy and does not shift in velocity as the broad line becomes blueshifted, showing that these photons
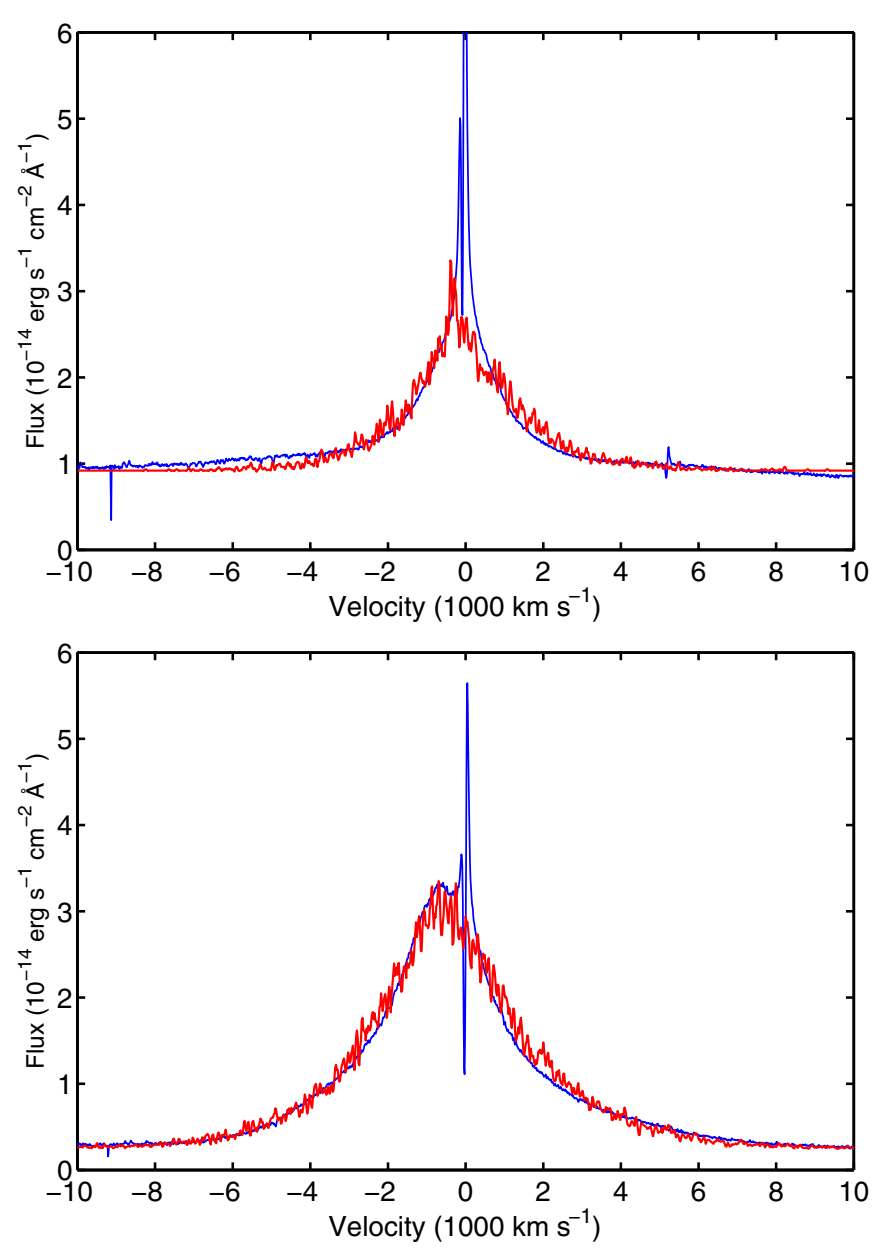

Figure 30. Electron scattering line profiles for day 31 (top) and day 222 (bottom). The observed profiles (from NOT) are in blue, and the calculated profiles from the Monte Carlo simulations are in red.

(A color version of this figure is available in the online journal.)

are not the dominant source for the scattering. The injected photons span a velocity range of $0-700 \mathrm{~km} \mathrm{~s}^{-1}$, as expected for radiative acceleration (see Section 4.4.3), which decreases the unscattered narrow peak, in agreement with the observations. The temperature is taken to decrease from $2 \times 10^{4} \mathrm{~K}$ to $1 \times 10^{4} \mathrm{~K}$, similar to what is found in detailed simulations of shocks (e.g., Figure 6 in Moriya et al. 2011), as well as from our photoionization calculations in Section 4.7. However, the profile is not sensitive to these assumptions.

As can be seen from the figure, we get excellent fits for both dates, except at zero velocity, where the P-Cygni profile from the CSM affects the profile. This confirms that electron scattering dominates the line formation. There is no need for two different velocity components in the broad lines as advocated by Zhang et al. (2012). The same conclusion is reached by Borish et al. (2014) for the day $36 \mathrm{~Pa} \beta$ line.

The fact that the line profile can be explained by electron scattering plus a linear velocity transformation is surprising. As was pointed out above, one expects a redshifted rather than blueshifted line profile if the expansion of the ejecta/CSM is spherically symmetric (see Fransson \& Chevalier 1989, Figure 6). Instead we propose that the $\mathrm{H} \alpha$ emission comes from a highly asymmetric, almost planar region expanding toward us (although possibly at some inclination relative to the LOS), as discussed in Section 4.7. 


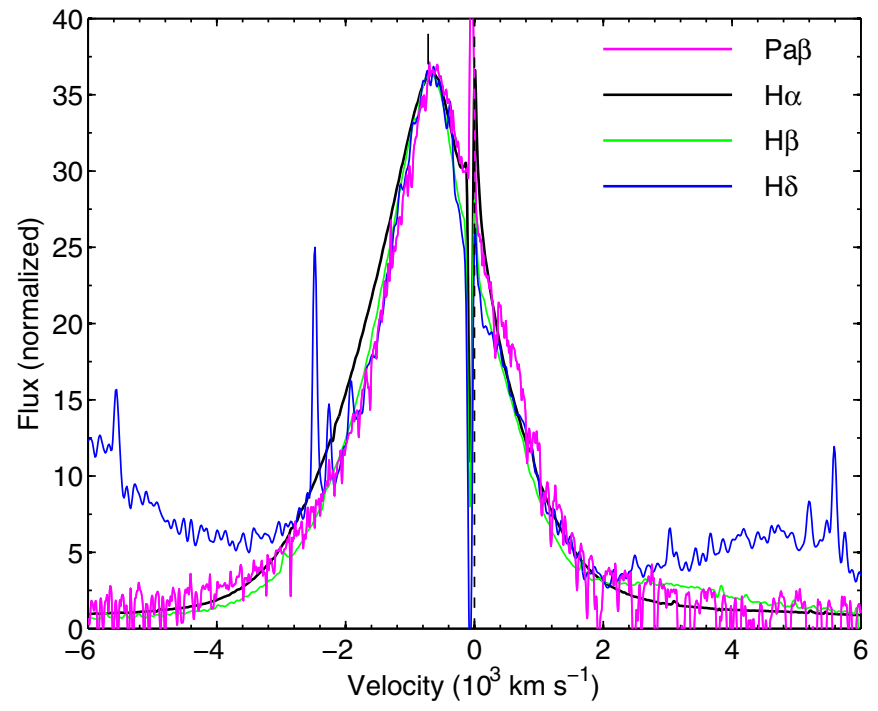

Figure 31. Comparison of the scaled line profiles of $\mathrm{H} \alpha, \mathrm{H} \beta, \mathrm{H} \delta$, and $\mathrm{Pa} \beta$ from the X-shooter spectrum from day 461 . The dashed line gives zero velocity, and the solid line gives the $-720 \mathrm{~km} \mathrm{~s}^{-1}$ blueshift of the $\mathrm{H} \alpha$ peak. Note the near coincidence of the peaks of all lines with that of $\mathrm{H} \alpha$.

(A color version of this figure is available in the online journal.)

\subsection{Origin of the Line Shifts}

\subsubsection{Dust Absorption from the Ejecta}

In Figure 29 we see a gradual shift of the $\mathrm{H} \alpha$ peak. We have already in Sections 4.2 and 4.3 argued against dust in the ejecta or at the reverse shock as a reason for this. Nevertheless, this has been discussed by several authors, so we consider additional arguments on this topic.

Smith et al. (2012b) analyze the line profiles during the first 236 days and discuss two different scenarios for the observed blueshift. From a comparison of the optical and nearIR hydrogen line profiles at $\sim 100$ days, they claim to see a wavelength-dependent asymmetry, which they interpret as a sign of wavelength-dependent extinction, indicative of dust formation in the postshock gas. However, Smith et al. also discuss electron scattering as an explanation for the line profile.

Maeda et al. (2013) argue for a dust origin based on the line shift, drop in luminosity, and IR excess. However, their analysis has several limitations, which we discuss based on our observations. The line shifts discussed by Maeda et al. are based on a single spectrum on day 513 with a limited $\mathrm{S} / \mathrm{N}$ for lines other than $\mathrm{H} \alpha$ (see their Figures 4 and 5). The line shifts derived from these observations are therefore highly uncertain and do not include the red wings of the lines. As we show in Figures 28 and 30 , we can get a satisfactory fit of both wings with an electron scattering profile.

Maeda et al. also claim to observe a systematic shift of the velocity with wavelength that is characteristic of dust extinction. In Figure 31 we compare the line profiles of our high $\mathrm{S} / \mathrm{N}$ $\mathrm{X}$-shooter spectrum for $\mathrm{H} \alpha, \mathrm{H} \beta, \mathrm{H} \gamma$, and $\mathrm{Pa} \beta$ at 461 days, an epoch not very different from that of Maeda et al. For these lines we have applied a smoothing of $\sim 50 \mathrm{~km} \mathrm{~s}^{-1}$ to the spectra and normalized the fluxes to the same peak flux for the broad component and the same "continuum" level in the range $\pm(6000-7000) \mathrm{km} \mathrm{s}^{-1}$. This is somewhat lower than for the determination of the continuum level of the $\mathrm{H} \alpha$ line in Figure 12, but it is necessary to avoid line contamination for $\mathrm{H} \beta$ and $\mathrm{Pa} \beta$. As seen in Figure 31, this is even more serious for $\mathrm{H} \gamma$, and we have therefore scaled this line to the level of the other lines at the peak and at the maximum velocity where the line dominates, $\sim 2000 \mathrm{~km} \mathrm{~s}^{-1}$. The high spectral resolution makes it possible to isolate the narrow P-Cygni component of the lines, which contaminates the low-resolution line profiles of Maeda et al. Both the spectral resolution and the $\mathrm{S} / \mathrm{N}$ are crucial for a reliable determination of the peak velocity as well as the general line profile. Although the day 461 spectrum is the best one in terms of $\mathrm{S} / \mathrm{N}$ and coverage to the NIR, we do not see any significant difference in the wavelength shifts for any other epochs. This includes the UV lines (Figures 14 and 15), which should be most sensitive to extinction.

As can be seen from the figure, there is no notable difference in the velocity of the broad lines. The peak of $\mathrm{H} \alpha$ is shifted by $\sim 720 \mathrm{~km} \mathrm{~s}^{-1}$ for this date. The $\mathrm{H} \delta, \mathrm{H} \beta, \mathrm{Pa} \beta$, and $\mathrm{He} \mathrm{I}$ $\lambda 10,830$ lines have their peaks at $640 \mathrm{~km} \mathrm{~s}^{-1}, 670 \mathrm{~km} \mathrm{~s}^{-1}$, $700 \mathrm{~km} \mathrm{~s}^{-1}$, and $690 \mathrm{~km} \mathrm{~s}^{-1}$, respectively. From variations of the smoothing we estimate an error of $\pm 50 \mathrm{~km} \mathrm{~s}^{-1}$. For $\mathrm{H} \alpha$, with a very high $\mathrm{S} / \mathrm{N}$, the error is $\lesssim 30 \mathrm{~km} \mathrm{~s}^{-1}$. We therefore do not find any significant trend of the peak velocity or line shape with wavelength.

Although they are distorted by the interstellar absorptions, one can also compare the Ly $\alpha$ line profile to the $\mathrm{H} \alpha$ and $\mathrm{H} \beta$ lines in Figure 14 for days 44, 107, and 621. It is clear that there is no significant difference in these in spite of the large wavelength difference and large time span. This is in contrast to the changing line profiles of the $\mathrm{Ly} \alpha, \mathrm{Mg}$ II, and $\mathrm{H} \alpha$ in $\mathrm{SN}$ 1998S (e.g., Figure 8 in Fransson et al. 2005), where such a difference was indeed seen, indicative of dust formed behind the reverse shock. The line profiles in SN 1998S were also very asymmetric. Thus we find that the main argument for ejecta dust in Maeda et al. is doubtful.

Another indicator of ejecta dust discussed by Maeda et al., the NIR excess, has already been discussed as the probable result of an echo. The third, the drop in the light curve, has a natural explanation in terms of the shock either exiting the circumstellar shell or transiting to a momentum-driven phase, as proposed by Ofek et al. (2014) and discussed in Section 4.5.

After this paper was first submitted Gall et al. (2014, in the following G14) published an analysis of the line shift based on dust absorption. In contrast to Andrews et al. (2011) and this paper, G14 interpret the NIR dust emission as well as the blueshifts as being caused by dust formed behind the reverse shock at an initial radius of $\sim 2 \times 10^{16} \mathrm{~cm}$. There are, however, a number of problems with this interpretation. First, it requires the shock to have reached this radius at their first observation, 26 days after peak or 66 days after first detection. This requires a shock velocity of $\sim 35,000 \mathrm{~km} \mathrm{~s}^{-1}$. To support this, G14 claim to see velocities up to $\sim 20,000 \mathrm{~km} \mathrm{~s}^{-1}$ from an assumed P-Cygni absorption in $\mathrm{H} \beta$ extending to this velocity. If this would be real, there would in that case also be an emission component up to a similar velocity, because the photosphere, assumed to be at $\sim 7500 \mathrm{~km} \mathrm{~s}^{-1}$, will only occult a small fraction of the ejecta. Such a broad emission component is not seen. The only way around this is to assume that the ejecta is extremely asymmetric. The P-Cygni absorptionis instead likely to be the result of contributions by weaker emission lines blueward of $\mathrm{H} \beta$. Further, there are no indications of similar high velocities from any other lines. In particular, the $\mathrm{Ly} \alpha$ line, being a resonance line, should show such absorption and emission if real. As seen in Figure 14, this is not the case. Nor do any of the other UV resonance lines show such a component (Figure 15). There are also other observations disfavoring similar high velocities. 
The analysis of the X-rays imply a velocity of $\lesssim 6000 \mathrm{~km} \mathrm{~s}^{-1}$ (Ofek et al. 2014). The only direct evidence for high expansion velocities comes from the NIR spectra, where the He I $\lambda 10,830$ line shows evidence for expansion velocities of $\sim 5500$ between 100 and 200 days toward us (Borish et al. 2014).

There are additional problems with the scenario in G14. If the emission lines would be coming from the cool dense shell and absorbed by the dust formed in this, the lines would be expected to have a boxy profile, not a symmetric profile peaked at low velocities. Boxy profiles are indeed seen in objects like SN 1993J (Matheson et al. 2000) and SN 1995N (Fransson et al. 2002), where the cool dense shell is believed to dominate the emission. Also, in objects where there are indeed strong indications for dust formation, like SN 1998S and SN 2006jc, the line profiles are less centrally peaked and more irregular (Leonard et al. 2000; Pozzo et al. 2004; Fransson et al. 2005; Smith et al. 2008b). As we have shown, the line profiles are instead well characterized as resulting from electron scattering. The mechanism behind the blueshift is not discussed in the G14 paper. It would, however, be surprising if this gave an intrinsically symmetric profile a simple shift in velocity, as we find, rather than a more irregular shape, given the expected clumping in a cold dense shell (Chevalier \& Blondin 1995).

Taking these results together, we therefore conclude that a cold dense shell at $\sim 35,000 \mathrm{~km} \mathrm{~s}^{-1}$ is unlikely and that the dust emission is instead coming from preexisting dust, as concluded by Andrews et al. (2011). If the shock velocity is much lower than $\sim 35,000 \mathrm{~km} \mathrm{~s}^{-1}$, the shock will be well inside the evaporation radius, even for the large grains G14 propose, as Table 9 and also the estimates in G14 show. Formation of the dust is then very difficult, as discussed before.

We are also surprised that G14 exclude the $\mathrm{H} \alpha$ line in their analysis, given that this is by far the highest $\mathrm{S} / \mathrm{N}$ line and therefore best suited for this kind of analysis. In addition, it should be noted that the asymmetry of $\mathrm{H} \alpha$ and other lines is sensitive to the assumed continuum level. A continuum subtraction has apparently not been done, as can be seen in Figure 5 of G14. The claims for a strong asymmetry in $\mathrm{H} \alpha$ are therefore doubtful. Instead, it can be seen from our Figure 10 that $\mathrm{H} \beta$ is considerably more complicated to analyze, with a number of interfering lines.

G14 further argue against preexisting dust based on the high dust temperature observed. It is not clear what this is based on, and on the contrary this is a natural consequence of dust evaporation by the strong flux from the $\mathrm{SN}$. The same conclusion is reached by Borish et al. (2014).

Taken together, we therefore think the results regarding dust formation and the derived properties of this in G14 are highly questionable.

Based on this and our earlier discussion, we exclude the dust alternative, and instead we believe that the line shifts are due to a bulk velocity of the scattering material. There are at least two possibilities for this.

\subsubsection{Gradual Contribution from Postshock Gas}

One alternative is that the velocity shift is caused by an increasing contribution of emission from the cooling gas behind the outgoing viscous shock. A possible scenario would be that at the first epochs the scattering optical depth in front of the shock is large enough that most of the line photons are emitted from unshocked gas with low velocities. As the optical depth decreases, an increasing contribution of the emitted photons will come from shocked gas behind the radiative forward shock. That this shock is indeed cooling is shown in Section 4.5. These photons will be scattered by electrons both in front of and behind the shock, producing the symmetric wings of the lines. There will therefore be a gradual shift from zero velocity to the velocity of the shock of the line profiles.

The main problem with this model is the high density in the postshock gas. The forward shock is expected to be radiative. The temperature immediately behind the shock will be $\sim 10^{8} \mathrm{~K}$, but it will cool down to $(1-2) \times 10^{4} \mathrm{~K}$. The compression behind the shock will therefore be a factor of $10^{3}-10^{4}$ in density, implying a density of $\gtrsim 10^{13} \mathrm{~cm}^{-3}$ where the gas is cool enough to emit $\mathrm{H} \alpha$ and other low-ionization lines. The calculations discussed in Section 4.7 show that at densities $\gtrsim 10^{11} \mathrm{~cm}^{-3}$ the emission is in LTE and thermalized, with a very low efficiency of conversion into line emission. Most radiation will emerge as a blackbody continuum. The large $\mathrm{H} \alpha$ luminosity is very difficult to explain in this scenario.

In principle, the $\mathrm{H} \alpha$ emission could come from the unshocked ejecta, where the density is lower. From the swept-up mass derived from the light curve (Equation (12) below), the column density of the cool shell is $\sim 10^{25} \mathrm{~cm}^{-2}$, meaning that all X-rays below $\sim 15 \mathrm{keV}$ will be absorbed in the shell. Only the reverse shock will therefore be able to ionize this region. The luminosity of the reverse shock is only $\sim 10 \%$ of the forward shock, so there will be a serious problem with the energy. Finally, it is not clear that a superposition of the emission from the preshock and postshock gas will result in the observed symmetric profiles.

Taken together, we believe that this explanation for the line shifts is unlikely.

\subsubsection{Radiative Acceleration}

A different alternative is that the line shift is the result of acceleration of the preshock gas by the extremely energetic radiation field from the shock and thermalized optical radiation from the ejecta and cool shell. This has been discussed earlier in different contexts (e.g., Chevalier 1976; Fransson 1982), but no conclusive observational evidence has been found. As a simple model, we consider an optically thin shell at a radius $r(t)$ illuminated from below by the radiation field of the $\mathrm{SN}$, having a luminosity $L(t)$. Assuming that we can consider the radiation as radially free streaming, which should be a reasonable approximation at small optical depths close to the surface, the velocity is given by

$$
V(t)=\frac{\kappa}{4 \pi c} \int_{0}^{t} \frac{L\left(t^{\prime}\right)}{r\left(t^{\prime}\right)^{2}} d t^{\prime} .
$$

For $\kappa$ we use the electron scattering opacity, $\sim 0.35$, applicable for an ionized medium with a $\mathrm{He} / \mathrm{H}$ ratio of 0.1 .

For the bolometric luminosity from the $\mathrm{SN}$, we use the result from Figure 6, shown on a linear scale in the upper panel of Figure 32. The total radiated energy from the SN (excluding the echo) was $\sim 6.5 \times 10^{50} \mathrm{erg}$. Using this energy and assuming a radius constant with time, we can estimate the final velocity as $V \approx 670\left(r / 3 \times 10^{15} \mathrm{~cm}\right)^{-2} \mathrm{~km} \mathrm{~s}^{-1}$. This estimate is, however, only approximate. The reason is that the hydrodynamic timescale of this gas is comparable to the length over which most of the energy is emitted, $t_{\text {hydro }}=$ $580\left(r / 3 \times 10^{15} \mathrm{~cm}\right) /\left(V / 700 \mathrm{~km} \mathrm{~s}^{-1}\right)$ days. The increase of the radius of the shell must therefore be taken into account when calculating the velocity. This is really a hydrodynamic problem, which includes the interaction of the shell with the surrounding 


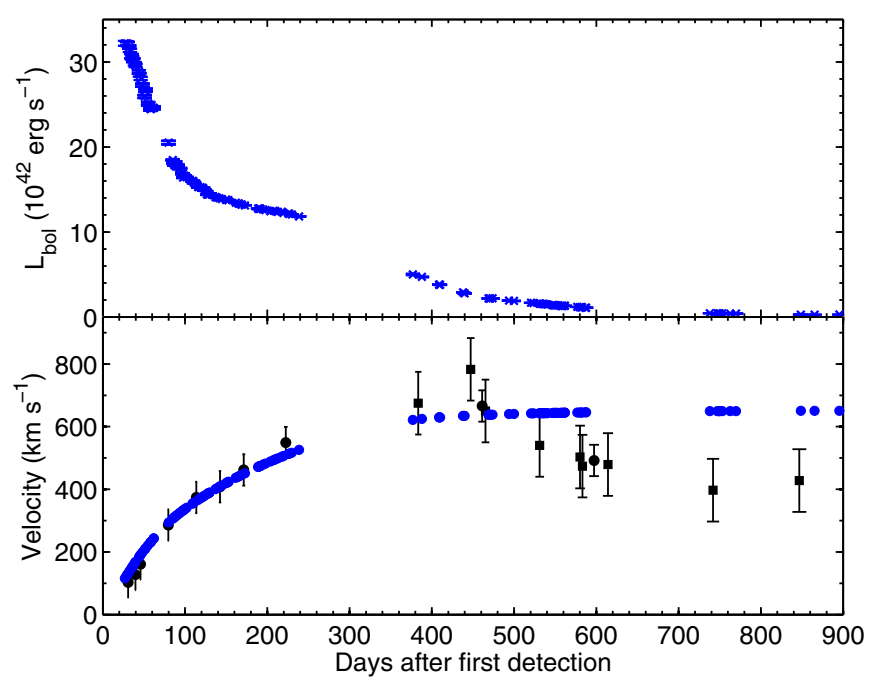

Figure 32. Upper panel: bolometric light curve from the $\mathrm{SN}$, excluding the IR dust echo, but including the UV and IR contributions from the direct SN emission. Note the linear luminosity scale in this figure, most relevant for the radiative acceleration, compared to the logarithmic one in Figure 3. Lower panel: the velocity shift found from $\mathrm{H} \alpha$ together with the velocity predicted from the acceleration by the $\mathrm{SN}$ radiation from Equation (1).

(A color version of this figure is available in the online journal.)

gas. We ignore the latter effects and treat the expansion as ballistic, calculating the radius as $r=\int_{t_{0}}^{t} v\left(t^{\prime}\right) d t^{\prime}+r_{0}$. The only free parameter is the initial radius, $r_{0}$, of the emitting shell, which gives the normalization of the velocity.

For the best fit we find $r_{0}=2.6 \times 10^{15} \mathrm{~cm}$, resulting in a velocity as a function of time shown by the blue dots in the lower panel of Figure 32. Given that there are uncertainties in the estimate of the bolometric luminosity, we find a good agreement with the observed velocity shift from Figure 29 and shown as black dots in Figure 32. At epochs later than 500 days, there is considerable scatter in the observed line shift, and it is difficult to judge if there is a slight decrease in the velocity, as may happen if there is a braking effect by the swept-up CSM. Apart from the general agreement with the evolution of the velocity, it is also interesting that the initial radius that gives the best fit, $r_{0}=2.6 \times 10^{15} \mathrm{~cm}$, is close to that which gives the best fit to the photospheric radius from the blackbody fitting of the SED at the early epochs, $R_{\text {phot }} \approx 3.2 \times 10^{15} \mathrm{~cm}$ (Table 5). The final radius after $\sim 900$ days is $7 \times 10^{15} \mathrm{~cm}$, and the velocity is $650 \mathrm{~km} \mathrm{~s}^{-1}$.

As we discussed in Section 4.3, an important constraint on the source of the $\mathrm{H} \alpha$ emission is the relation of the narrow unscattered line and the scattered wings, with the fraction of the former approximately given by $\propto \exp \left(-\tau_{\mathrm{e}}\right)$ for a planar geometry. Because the typical optical depth required for the wings is $2-5$, depending on the temperature, this would in general give a strong narrow line. This is indeed observed at early time, but it disappears at later epochs at the same time as the blueshift starts (Figure 12). In this scenario, this may be explained by the fact that the source of the $\mathrm{H} \alpha$ photons become increasingly spread out in velocity, reflecting the local, accelerated velocity, but now spanning an increasing velocity range. The peak flux will decrease with the increasing velocity of this material, as observed. One therefore obtains a consistency check between the flux of the unscattered emission and the velocity shift. Note that the narrow CSM line most likely comes from a different, more distant component than the broad lines, based on its constant velocity and different flux evolution (Figure 18).
The velocity of this radiatively accelerated material is considerably lower than the $\sim 3000 \mathrm{~km} \mathrm{~s}^{-1}$ one infers from the $\mathrm{X}$-rays. After a time of $t \approx r_{0} /\left(V_{s}-V_{\text {acc }}\right) \approx 1.0\left(r_{0} / 2.6 \times\right.$ $\left.10^{15} \mathrm{~cm}\right) /\left(V_{s} / 650 \mathrm{~km} \mathrm{~s}^{-1}-1\right) \mathrm{yr}$, the shock will sweep up the accelerated shell. For this not to happen too early, the shock velocity interior to the $\mathrm{H} \alpha$-emitting shell has to be below $\sim 1500 \mathrm{~km} \mathrm{~s}^{-1}$, depending on the velocity of the accelerated shell, corrected for the LOS angle. As discussed earlier, a reason for a lower shock velocity may be that the shell is anisotropic with the highest column density where the broad lines arise. The shock velocity will then be lower than that inferred from the Xrays, which are likely to come from directions of lower column density (see the next section).

The mass of the accelerated gas does not need to be large and is likely to be small. The scattering layer only has to have a $\tau_{\mathrm{e}} \gtrsim 1$, corresponding to $\sim 0.14\left(r / 3 \times 10^{15} \mathrm{~cm}\right)^{2}(\Omega / 4 \pi) M_{\odot}$, where $r$ is the radius of the shell and $\Omega$ the solid angle of the shell. To accelerate it to $\sim 1000 \mathrm{~km} \mathrm{~s}^{-1}$ only takes $\sim 1.5 \times 10^{48}(\Omega / 4 \pi) \mathrm{erg}$, which is small compared to the total radiated energy. Most of the mass in the CSM is instead in the optically very thick region inside the accelerated layer.

\subsection{Shock Breakout, Circumstellar Interaction, and Energy Source}

The high luminosity, $\sim 3 \times 10^{43} \mathrm{erg} \mathrm{s}^{-1}$ at maximum, and the large total radiated energy, $\gtrsim 6.5 \times 10^{50}$ erg (Section 3.2), require an efficient conversion of kinetic to radiative energy. In Section 4.2 we argued that the flux from the optical and NIR up to $\sim 350$ days is dominated by the direct flux from the $\mathrm{SN}$, and most of the NIR flux is from the echo after this epoch. We now discuss the energetics in terms of the shock propagation through a dense envelope.

The shock breakout for extended SNe has been discussed by several authors (Falk \& Arnett 1977; Grasberg \& Nadyozhin 1987; Moriya et al. 2011, 2013; Chevalier \& Irwin 2011; Ginzburg \& Balberg 2012). For large optical depths the shock will be radiation dominated with a width $\tau_{\mathrm{e}} \sim c / v$ (Weaver 1976). Chevalier \& Irwin (2011) estimate the diffusion timescale of the CSM, assumed to have $\rho \propto r^{-2}$, as

$$
\begin{aligned}
t_{\mathrm{diff}} \approx & 6.6\left(\frac{\dot{M}}{0.1 M_{\odot} \mathrm{yr}^{-1}}\right)\left(\frac{u_{\mathrm{w}}}{100 \mathrm{~km} \mathrm{~s}^{-1}}\right)^{-1} \\
\times & \left(\frac{\kappa}{0.34 \mathrm{~cm}^{2} \mathrm{~g}^{-1}}\right) \text { days. }
\end{aligned}
$$

Here $\dot{M}$ is the mass-loss rate, $u_{w}$ is the wind velocity, and $\kappa$ is the opacity. For the mass-loss rates we derive below, this is likely to be short for the epochs of interest here. As the shock approaches the surface at $\tau_{\mathrm{e}} \lesssim c / v$, a viscous shock will form (for a discussion, see Chevalier \& Irwin 2011). If the circumstellar density is high, the viscous shock will be radiative, implying an efficient conversion of kinetic to radiative energy.

The cooling time for the forward shock is given by $t_{\text {cool }}=$ $3 k T / n_{\mathrm{e}} \Lambda$, where the cooling rate can be approximated by $\Lambda=2.4 \times 10^{-23} T_{8}^{0.5} \mathrm{erg} \mathrm{s}^{-1} \mathrm{~cm}^{3}$ for temperatures above $\sim 2 \times 10^{7} \mathrm{~K}$. For close to solar abundances, the shock temperature is given by $T_{\mathrm{e}}=1.2 \times 10^{8}\left(V_{\mathrm{s}} / 3000 \mathrm{~km} \mathrm{~s}^{-1}\right)^{2} \mathrm{~K}$. For an ejecta profile given by $\rho_{\text {ejecta }} \propto r^{-n}$, the shock radius is

$R_{\mathrm{s}}=9.47 \times 10^{15} \frac{(n-2)}{(n-3)}\left(\frac{V_{\mathrm{s}, 320}}{3000 \mathrm{~km} \mathrm{~s}^{-1}}\right)\left(\frac{t}{\text { years }}\right)^{(n-3) /(n-2)}$ 


$$
=1.23 \times 10^{16}\left(\frac{V_{\mathrm{s}, 320}}{3000 \mathrm{~km} \mathrm{~s}^{-1}}\right)\left(\frac{t}{\text { years }}\right)^{0.82} \mathrm{~cm},
$$

for $n=7.6$ as we find below. Based on the shock velocity derived by Ofek et al. (2014), we scale this and the following estimates to a shock velocity at 320 days, $V_{\mathrm{s}, 320}$, of $3000 \mathrm{~km} \mathrm{~s}^{-1}$. Note that this velocity may vary with the direction if the massloss rate is anisotropic (see below), as we argue. This gives a density immediately behind the forward shock of

$$
\begin{aligned}
n_{\mathrm{s}, \mathrm{e}}= & 8.1 \times 10^{8}\left(\frac{\dot{M}}{0.1 M_{\odot} \mathrm{yr}^{-1}}\right)\left(\frac{u_{\mathrm{w}}}{100 \mathrm{~km} \mathrm{~s}^{-1}}\right)^{-1} \\
& \times\left(\frac{V_{\mathrm{s}, 320}}{3000 \mathrm{~km} \mathrm{~s}^{-1}}\right)\left(\frac{t}{\text { years }}\right)^{-1.64} \mathrm{~cm}^{-3}
\end{aligned}
$$

which leads to

$$
\begin{aligned}
t_{\text {cool }}= & 26.6\left(\frac{\dot{M}}{0.1 M_{\odot} \mathrm{yr}^{-1}}\right)^{-1}\left(\frac{u_{\mathrm{w}}}{100 \mathrm{~km} \mathrm{~s}^{-1}}\right) \\
& \times\left(\frac{V_{\mathrm{s}, 320}}{3000 \mathrm{~km} \mathrm{~s}^{-1}}\right)^{3}\left(\frac{t}{\text { years }}\right)^{1.46} \text { days. }
\end{aligned}
$$

Therefore, the forward shock should be radiative for several years as long as the shock is in the high-density shell. Further, assuming that the ingoing X-ray flux from the shock is thermalized in the ejecta and cool shell behind the shock, the blackbody luminosity is given by $(1 / 4) \dot{M} V_{\mathrm{s}}^{3} / u_{\mathrm{w}}$. An equal amount will partly be absorbed and ionize the preshock gas and partly escape as the observed X-ray flux. Using this luminosity for the radiation density, the ratio of Compton to free-free cooling is then

$$
\frac{P_{\text {Compton }}}{P_{\text {free-free }}}=7.4 \tau_{e}\left(\frac{V_{\mathrm{s}}}{10^{4} \mathrm{~km} \mathrm{~s}^{-1}}\right)^{4}
$$

(Chevalier \& Irwin 2012). Therefore, for shocks with velocity below $\sim 5000 \mathrm{~km} \mathrm{~s}^{-1}$, free-free cooling dominates, unless the optical depth is very high.

For a general circumstellar density given by $\rho=$ $\dot{M} /\left(4 \pi u_{\mathrm{w}} r_{0}^{2}\right)\left(r_{0} / r\right)^{s}$, the ejecta velocity at the shock depends on the mass-loss rate and time as

$$
V_{\mathrm{s}} \propto\left(\frac{\dot{M}}{v_{\mathrm{w}}}\right)^{-1 /(n-s)} t^{-(3-s) /(n-s)},
$$

(e.g., Fransson et al. 1996), assuming the interaction can be described by a similarity solution. If the mass-loss rate is a function of polar angle, $V_{\mathrm{s}}$ will therefore depend on angle as well. For a radiative shock we find for the luminosity

$$
\begin{aligned}
L=2 \pi \rho V_{\mathrm{s}}^{3} r^{2}= & \frac{1}{2} \frac{\dot{M}}{u_{\mathrm{w}}}\left(\frac{r_{0}}{r}\right)^{s-2} V_{\mathrm{s}}^{3} \\
& \propto\left(\frac{\dot{M}}{u_{\mathrm{w}}}\right)^{(n-5) /(n-s)} t^{-[15+s(n-6)-2 n] /(n-s)} .
\end{aligned}
$$

For $s=2$ we obtain $L \propto t^{-3 /(n-2)}$.

The fit of Equation (9) to the data in Figure 6 implies a value of $n=7.6$ for $s=2$, as shown by the dashed line in this figure. The break at $\sim 320$ days in the light curve can either be caused by the breakout of the shock through the dense shell, or as a result of a transition to a momentum-conserving phase, occurring when the swept-up mass is comparable to the ejecta mass (Ofek et al. 2014). The latter explanation has, however, been contested by Moriya (2014), who find that the transition to the momentum-driven phase gives too smooth and slow a decrease of the luminosity. As we discuss in Section 4.7, the breakout in a bipolar shell may be compatible with this.

For $s=2$ (consistent with the X-ray light curve, see below) we get

$$
\begin{aligned}
L= & 8.51 \times 10^{42}\left(\frac{\dot{M}}{0.1 M_{\odot} \mathrm{yr}^{-1}}\right)\left(\frac{u_{\mathrm{w}}}{100 \mathrm{~km} \mathrm{~s}^{-1}}\right)^{-1} \\
& \times\left(\frac{V_{\mathrm{s}, 320}}{3000 \mathrm{~km} \mathrm{~s}^{-1}}\right)^{3}\left(\frac{t}{320 \text { days }}\right)^{-0.537} \mathrm{erg} \mathrm{s}^{-1}
\end{aligned}
$$

For a luminosity at the break at $\sim 320$ days of $9.33 \times 10^{42} \mathrm{erg} \mathrm{s}^{-1}$ we then find

$$
\dot{M}=0.11\left(\frac{u_{\mathrm{w}}}{100 \mathrm{~km} \mathrm{~s}^{-1}}\right)\left(\frac{V_{\mathrm{s}, 320}}{3000 \mathrm{~km} \mathrm{~s}^{-1}}\right)^{-3} M_{\odot} \mathrm{yr}^{-1}
$$

The total mass swept up is then

$$
\Delta M=\dot{M} \frac{V_{\mathrm{s}}}{u_{\mathrm{w}}} t=2.89\left(\frac{V_{\mathrm{s}, 320}}{3000 \mathrm{~km} \mathrm{~s}^{-1}}\right)^{-2} M_{\odot} .
$$

Both the mass loss and total mass are sensitive to the expansion velocity of the ejecta. In this estimate we assume that the ejecta with a velocity of $\sim 3000 \mathrm{~km} \mathrm{~s}^{-1}$ dominates the energy input to the optical light curve. In reality, there should be a range in velocities, and there is therefore a considerable uncertainty in this estimate. The fact that we observe both hard $\mathrm{X}$-rays with a temperature corresponding to a shock velocity of $\sim 3000 \mathrm{~km} \mathrm{~s}^{-1}$ and with a column density corresponding to an electron scattering depth of $\tau_{\mathrm{e}} \lesssim 1$, as well as high column density gas with $\tau_{\mathrm{e}} \gtrsim 3$, argues for a highly anisotropic CSM. This is further strengthened by the blue shoulder seen by Borish et al. (2014) in the He I $\lambda 10,830$ line in the span $\sim 100-200$ days after explosion. The velocity of this decreases during this interval from $\sim 6000 \mathrm{~km} \mathrm{~s}^{-1}$ to $\sim 5000 \mathrm{~km} \mathrm{~s}^{-1}$, indicating deceleration of this material. The shock velocity in the X-ray obscured regions may, however, be considerably lower (Equation (8)). The mass-loss rate above, as well as the total mass lost, should therefore be considered as lower limits. Note, however, that while the mass-loss rate scales with the velocity of the CSM, the total mass lost is independent of this parameter.

Ofek et al. (2014) find a considerably higher mass-loss rate of $\sim 0.8 M_{\odot} \mathrm{yr}^{-1}$. The main reason for this is that they are scaling to a higher wind velocity of $u_{\mathrm{w}}=300 \mathrm{~km} \mathrm{~s}^{-1}$, while we find $u_{w} \approx 100 \mathrm{~km} \mathrm{~s}^{-1}$. They also assume an efficiency of $\sim 0.25 \mathrm{in}$ converting the shock energy into the observed luminosity. The reason for this is not clear. Another important difference is that they assume an ejecta profile $n=10$, close to that found by Matzner \& McKee (1999) for the ejecta of a radiative envelope, while we derive $n \approx 7.6$ from our bolometric light curve. Their larger value of $n$ is a result of the flatter light curve they derive from the $R$ band only, $L \propto t^{-0.38}$ compared to our $L \propto t^{-0.54}$ and using $L \propto t^{-3 /(n-2)}$ (Equation (9)).

Even considering the above uncertainties, the mass-loss rate and the total mass lost are very large, but as discussed in Section 4.1, these are of the same magnitude as that inferred from the CSM of local LBVs, like Eta and AG Carinae. We discuss the implications of this further in Section 4.7, but we first place it in relation to other Type IIn SNe. 


\subsection{Comparison to Other Type IIn Supernovae}

Although interesting as a case by itself, it is at least as interesting to put SN 2010jl into the context of other Type IIn SNe. There have been several Type IIn SNe that show similarities to SN 2010jl to various degrees, although in most cases these events are less extreme in terms of luminosity or total radiated energy. As we show in this section, most of these, SNe $1995 \mathrm{~N}, 1998 \mathrm{~S}, 2005 \mathrm{ip}$, and 2006jd, although observationally quite different, are related to SN 2010j1, and two others recently discussed, SN 2009ip and SN 2010mc, while having some properties in common, show major differences from SN 2010jl.

SN 1998S is one of the best-observed Type IIn SNe and is interesting as a less extreme case of a Type IIn SN. Initially it showed typical Type IIn signatures with narrow symmetric lines (Leonard et al. 2000; Fassia et al. 2001) dominated by electron scattering of $\mathrm{H}$ and $\mathrm{He}$ I lines (Chugai 2001). The symmetric lines disappeared after about a week, and instead broad P-Cygni profiles with an expansion velocity of $\sim 7000 \mathrm{~km} \mathrm{~s}^{-1}$ appeared. Later optical spectra at $\gtrsim 70$ days showed increasingly box-like profiles typical of circumstellar interaction, with $\mathrm{H} \alpha$ by far the strongest and a steep Balmer decrement. Spectra later than a year showed a strong suppression of the red wing of $\mathrm{H} \alpha$, as well as $\operatorname{Ly} \alpha$, indicating dust formation in the ejecta or reverse shock (Leonard et al. 2000; Pozzo et al. 2004; Fransson et al. 2005). As was remarked earlier, these line profiles were very different from those in SN 2010jl.

High-velocity ejecta profiles of $\mathrm{H} \alpha$ were also seen for $\mathrm{SN}$ 1995N (Fransson et al. 2002), SN 2005ip, and 2006jd (Smith et al. 2009; Stritzinger et al. 2012). For the latter two, maximum ejecta velocities of $16,000-18,000 \mathrm{~km} \mathrm{~s}^{-1}$ were seen at early times (Smith et al. 2009; Stritzinger et al. 2012). For SN $2006 j$ a high-velocity wing at $\sim 7000 \mathrm{~km} \mathrm{~s}^{-1}$ could be seen even at 1540 days. In addition, an intermediate-velocity component with an FWHM of $\sim 1800 \mathrm{~km} \mathrm{~s}^{-1}$ was seen in both SNe. There were no strong indications of electron scattering wings in these $\mathrm{SNe}$, although this may have contributed to the intermediate component or faded away before their discovery, as for SN 1998S.

In the last HST spectra of SN 1998S, broad features from O I $\lambda \lambda 1302,1356$ were seen, indicating processed material (Fransson et al. 2005). Recently, Mauerhan \& Smith (2012) found that at $14 \mathrm{yr}$ the spectrum of SN 1998S was dominated by strong lines of [O I-III]. This shows that the reverse shock has now propagated close to the core of the SN and that newly processed gas is dominating the spectrum. There is therefore no doubt that a core collapse has taken place. For SN 2010j1 this phase has not yet occurred.

Hard X-rays with $k T \sim 10 \mathrm{keV}$ and a luminosity of $\sim(5-8) \times$ $10^{39} \mathrm{erg} \mathrm{s}^{-1}$ were observed for SN 1998S two to three years after explosion (Pooley et al. 2002). The X-ray light curve of SN 2006jd stayed nearly flat with an unabsorbed luminosity of (3-4) $\times 10^{41} \mathrm{erg} \mathrm{s}^{-1}$ in the span 400-1600 days (Chandra et al. 2012a), lower than for SN 2010jl. Also, the column densities of these $\mathrm{SNe}, \lesssim 1.5 \times 10^{21} \mathrm{~cm}^{-2}$, were considerably lower than for SN 2010jl and did not show any strong time evolution as for SN 2010jl.

High-resolution spectra of SN 1998S by Fassia et al. (2001) exhibited a low-velocity P-Cygni line with a velocity of $40-50 \mathrm{~km} \mathrm{~s}^{-1}$ and a higher velocity extension with velocity $350 \mathrm{~km} \mathrm{~s}^{-1}$. The low-velocity component argues for a red supergiant progenitor. The origin of the higher velocity component is not clear. Variations of the wind velocity are also seen in LBVs and $\mathrm{S}$ Doradus stars, and such a progenitor is probably not excluded. In the case of SN 2005ip, the narrow component was only marginally resolved with FWHM $\sim 120 \mathrm{~km} \mathrm{~s}^{-1}$ (Smith et al. 2009).

The very different optical spectra of SN 1998S and the other mentioned SNe compared to SN 2010jl can naturally be explained as a result of the different CSM densities and shock velocities. The mass-loss rate of SN 1998S was from late observations estimated to be quite modest by Type IIn standards, $\sim 2 \times 10^{-5} \quad M_{\odot} \mathrm{yr}^{-1}$ (Fassia et al. 2001). The time of optical depth unity to electron scattering is for a steady wind (for simplicity assumed to extend to infinity) given by

$$
\begin{aligned}
t\left(\tau_{\mathrm{e}}=1\right)= & \frac{\kappa_{\mathrm{T}} \dot{M}}{4 \pi u_{\mathrm{w}} V_{\mathrm{s}}} \\
= & 680\left(\frac{\dot{M}}{0.1 M_{\odot} \mathrm{yr}^{-1}}\right)\left(\frac{u_{\mathrm{w}}}{100 \mathrm{~km} \mathrm{~s}^{-1}}\right)^{-1} \\
& \times\left(\frac{V_{\mathrm{s}}}{3000 \mathrm{~km} \mathrm{~s}^{-1}}\right)^{-1} \text { days }
\end{aligned}
$$

where we have scaled the parameters to SN 2010j1. Note, however, that in Section 4.5 we argue that $V_{\mathrm{s}}$ is considerably lower in the directions we observe. For SN 1998 S with $\dot{M} \approx$ $2 \times 10^{-5} M_{\odot} \mathrm{yr}^{-1}, u_{\mathrm{w}} \approx 50 \mathrm{~km} \mathrm{~s}^{-1}$, and $V_{\mathrm{s}} \approx 7000 \mathrm{~km} \mathrm{~s}^{-1}$, we find that $t\left(\tau_{\mathrm{e}}=1\right) \approx 0.1$ days. The fact that electron scattering wings were observed for several days argues for a denser shell at $\sim 10^{15} \mathrm{~cm}$ from the progenitor with $\dot{M} \approx$ $3 \times 10^{-3}\left(u_{\mathrm{w}} / 10 \mathrm{~km} \mathrm{~s}^{-1}\right) M_{\odot} \mathrm{yr}^{-1}$ (Chugai 2001).

Although there are uncertainties in these quantities, one can therefore conclude that the fast disappearance of the electron scattering wings and the transparency to the processed core in SN $1998 S$ (and the other discussed medium-luminosity Type IIns) compared to SN 2010jl is consistent with the much lower mass-loss rate of the former. Because the X-ray column density is proportional to the electron-scattering optical depth, this also explains the much lower X-ray column densities for these $S N e$. This argument also applies to the even higher luminosity "superluminous" Type II SNe (e.g., Gal-Yam 2012).

The UVOIR luminosity of SN 1998S was at the peak $\sim 2 \times 10^{43} \mathrm{erg} \mathrm{s}^{-1}$, but a blackbody fit gave a considerably higher luminosity of $\sim 6 \times 10^{43} \mathrm{erg} \mathrm{s}^{-1}$ (Fassia et al. 2000). This is similar to the peak luminosity of SN 2010jl. The initially much higher effective temperature, $\sim 18,000 \mathrm{~K}$, compared to $\sim 7300 \mathrm{~K}$ for SN 2010jl (Zhang et al. 2012), however, had the consequence that the $R$-band magnitude at maximum was only $\sim-19.1$, compared to $\sim-20.0$ for SN 2010j1. A main difference compared to SN 2010jl was that the decay was nearly exponential up to $\sim 100$ days with a fast decay rate of $\sim 25$ days. Including only the UVOIR luminosity, we estimate an uncertain total energy of $\sim 1.1 \times 10^{50} \mathrm{erg}$. Using instead the bolometric luminosity from the blackbody fits by Fassia et al., one gets a factor of two to three higher radiated energy.

As Pozzo et al. (2004) argue, the early light curve is likely to be dominated by the released shock energy, and the main reason for the high initial luminosity was the large extent of the progenitor, as indicated by the dense CSM during the first days, discussed above. The fast decay and the lower radiated total energy, however, indicate a lower total mass of the CSM compared to SN 2010jl. Chugai (2001) estimate a total mass of $\sim 0.1 M_{\odot}$ in the CSM of SN 1998S, $\sim 2$ orders of magnitude smaller than for SN 2010jl. 
SN 2005ip had a moderate peak luminosity for a Type IIn SN, $\sim 4 \times 10^{42} \mathrm{erg} \mathrm{s}^{-1}$ (Smith et al. 2009; Stritzinger et al. 2012). It then decayed on roughly the ${ }^{56}$ Co timescale to a nearly constant level at $\sim 200$ days, dominated by the NIR. The optical luminosity during this plateau phase was $\sim 2.5 \times 10^{41} \mathrm{erg} \mathrm{s}^{-1}$, decaying slowly as $t^{-0.3}$ from $\sim 200$ to $\gtrsim 1600$ days. From blackbody fits, Stritzinger et al. find a luminosity of $\sim 8 \times 10^{41} \mathrm{erg} \mathrm{s}^{-1}$ for the NIR warm component. Stritzinger et al. also discuss optical and IR observations of another interesting Type IIn, SN 2006jd. This SN had a similar early luminosity but considerably higher optical luminosity in the plateau phase, $\sim 8 \times 10^{41} \mathrm{erg} \mathrm{s}^{-1}$, and nearly constant from $\sim 200$ to $\gtrsim 1600$ days.

From Stritzinger et al. (2012) we estimate the total radiated energy of SN 2005 ip to be $\sim 4 \times 10^{49}$ erg from the photospheric emission and $\sim 7 \times 10^{49} \mathrm{erg}$ in the dust component, and for SN 2006jd to be $\sim 10^{50}$ erg from the photospheric emission and $\sim 2 \times 10^{50} \mathrm{erg}$ in the dust component. Although the luminosity and energy are high, both of these $\mathrm{SNe}$ are therefore considerably less extreme in terms of mass loss and degree of interaction compared to SN 2010j1.

An independent indicator of strong mass loss is the large observed $\mathrm{N} / \mathrm{C}$ ratio, characteristic of $\mathrm{CNO}$ processed material in the CSM. This is consistent with the fact that high $\mathrm{N} / \mathrm{C}$ ratios have been observed for all Type IIn, IIb, and IIL SNe observed with HST: SN 1979C, 1993J, 1995N, 1998S, and SN 2010j1 (Section 4.1).

A common feature of all these $\mathrm{SNe}$ is also the presence of high-ionization lines from the CSM. Ultraviolet spectra of SN 1998S from day 28 to day 485 showed increasingly strong lines from C III-IV and N III-V originating in the CSM. Also, SN 2005ip and 2006jd revealed a number of narrow highionization lines from the CSM. For SN 2006jd, this included lines from [Fe X-XI] and [Ar X], and SN 2005ip showed even higher ionization lines, including [Fe XIV] $\lambda 5302.9$ and [Ar XIV] $\lambda 4412.3$ (Smith et al. 2009). High-ionization lines were also seen in SN $1995 \mathrm{~N}$, which showed a spectrum very similar to SN 2006jd (Fransson et al. 2002). We note that Hoffman et al. (2008) observed similar high-ionization coronal lines in the Type IIn SN 1997eg. Nearly all of these SNe had a strong X-ray flux. The connection between this and the presence of circumstellar lines therefore indicates that the latter are excited by the X-rays in most of these cases. Conversely, the presence of circumstellar lines may be used as a diagnostic of a strong X-ray flux.

Near-IR spectra and photometry of SN 1998 S by Fassia et al. (2000) revealed a dust excess already at 136 days. Later observations by Pozzo et al. (2004) showed a comparatively hot dust spectrum in the first observations, with a dust temperature $\sim 1000-1250 \mathrm{~K}$ at $\sim 1 \mathrm{yr}$, decreasing to $\sim 750-850 \mathrm{~K}$ at 1198 days. Pozzo et al. argue for a dust echo for the first epochs, while the later emission may have an origin in the condensed dust in the cool dense shell. In contrast to SN 2010jl, there is no problem with dust evaporation at the shock radius for epochs later than $\sim 1 \mathrm{yr}$ because the bolometric luminosity from the SN was then $\lesssim 10^{41} \mathrm{erg} \mathrm{s}^{-1}$ (Pozzo et al. 2004). The dust evaporation radius is then $(1-2) \times 10^{16} \mathrm{~cm}$ (Table 9), and the shock radius is $\sim 3 \times 10^{16} \mathrm{~cm}$ for a shock velocity of $10^{4} \mathrm{~km} \mathrm{~s}^{-1}$.

Also, the Type Ibn SN 2006jc showed evidence for dust formation behind the reverse shock (Smith et al. 2008b; Mattila et al. 2008). Even at maximum, the luminosity of this SN was $\lesssim 10^{42} \mathrm{erg} \mathrm{s}^{-1}$, declining to $\lesssim 2 \times 10^{41} \mathrm{erg} \mathrm{s}^{-1}$ later than 100 days (Mattila et al. 2008). The expansion velocity was estimated to be $\gtrsim 8000 \mathrm{~km} \mathrm{~s}^{-1}$, indicating a shock radius of $\gtrsim 7 \times 10^{15} \mathrm{~cm}$ at 100 days. The high dust temperature, $\sim 1800 \mathrm{~K}$ at maximum, argues for carbon dust. From Table 9 we then find a dust evaporation radius of $\sim 5.6 \times 10^{15} \mathrm{~cm}$ at 100 days for carbon dust with a size of $1 \mu \mathrm{m}$. Because of the much lower luminosity compared to SN 2010jl, dust formation behind the reverse shock is therefore possible.

Gerardy et al. (2002) discuss NIR photometry and spectra for several Type IIn SNe. The best observed of these is SN $1995 \mathrm{~N}$ with NIR observations from 730 to 2435 days after explosion. During the first observations, the NIR luminosity was $\sim(7-10) \times 10^{41} \mathrm{erg} \mathrm{s}^{-1}$, depending on the assumed dust emissivity. It then slowly decayed by a factor of $\sim 10$ at the time of the last observations. The dust temperature was 700-900 K during most of the evolution. The other SNe observed also showed similar high temperatures. From the fading of the red wing of the $\mathrm{H} \alpha$ line in $\mathrm{SN} 1995 \mathrm{~N}$, late dust formation at an age of $\sim 1000$ days was indicated (Fransson et al. 2002).

Evidence for dust formation for SN 2005ip was first presented for this SN based on NIR photometry by Fox et al. (2009). Further observations in the NIR and with Spitzer showed evidence for two dust components with different temperatures (Fox et al. 2010). Fox et al. argue that the hot component comes from newly formed dust in the ejecta or reverse shock, and the cool emission comes from preexisting dust heated by the late circumstellar interaction. Independent evidence for dust came from line profile asymmetries (Smith et al. 2010a).

Both SN 2005ip and 2006jd showed strong IR excesses already shortly after the explosion. The fit to the warm components reveals for both $\mathrm{SNe}$ a maximum dust temperature of $\sim 1600 \mathrm{~K}$ at $100-200$ days, decreasing to $\sim 1000 \mathrm{~K}$ at 1000 days. The luminosity of the dust component of SN 2006jd increased to $\sim 3 \times 10^{42} \mathrm{erg} \mathrm{s}^{-1}$ at $\sim 500$ days and then decayed slowly to $\sim 5 \times 10^{41} \mathrm{erg} \mathrm{s}^{-1}$ at $\sim 1700$ days. The fraction of the bolometric luminosity in this warm component increased already at $\sim 100$ days to $\sim 80 \%$ and to even higher values at later times. SN 2005ip showed a similar behavior, although the luminosity of the warm component was only $\sim 5 \times 10^{41} \mathrm{erg} \mathrm{s}^{-1}$ and at a more constant level.

While we believe that an echo from preexisting dust may dominate the NIR emission in SN 2010j1, the other discussed mechanisms may well be important for other Type IIn SNe. All of these are physically plausible, and their relative importance depends on the specific case in terms of progenitor mass-loss rate, dust shell geometries, $\mathrm{SN}$ luminosity, including the $\mathrm{X}$-rays, and viewing direction.

Besides the discussed SNe, two recent Type IIn SNe, SN 2009ip (Smith et al. 2010b; Foley et al. 2011; Pastorello et al. 2013; Mauerhan et al. 2013) and SN 2010mc (Ofek et al. 2013), have received considerable attention. Both $\mathrm{SNe}$ showed minor outbursts with $M_{\mathrm{R}} \sim-14-15$ before the last major eruption. The main outburst had an absolute magnitude of $M_{R} \sim-18$, corresponding to a luminosity of $\sim(5-8) \times 10^{42} \mathrm{erg} \mathrm{s}^{-1}$, a factor of 4-6 lower than SN 2010jl. Another difference is that the flux decayed considerably faster during the first $\sim 60$ days, with an e-folding decay timescale of $\sim 20$ days. From the bolometric light curve we estimate a total radiated energy of $\sim 2 \times 10^{49} \mathrm{erg}$ for SN 2009ip and a similar energy for SN 2010mc. This is more than an order of magnitude lower than what we estimate for SN 2010jl.

Both narrow lines with broad electron scattering wings and broad ejecta lines were seen. Pastorello et al. (2013) stress the important observation that for SN 2009ip, already in the minor outbursts a year before the most recent large outburst, 
expanding material at a velocity of $\sim 12,500 \mathrm{~km} \mathrm{~s}^{-1}$ was seen. It is therefore clear that high velocities are not a unique signature of a core collapse, but they can also occur in stages before this. In connection to the 2012 September eruption, both an electron scattering profile with FWHM $\sim 550-800 \mathrm{~km} \mathrm{~s}^{-1}$ and a broad absorption extending to $14,000-15,000 \mathrm{~km} \mathrm{~s}^{-1}$ were seen.

Based on the large velocities and other arguments, Mauerhan et al. (2013) propose a core collapse scenario, which is, however, challenged by Pastorello et al. (2013). From the fact, mentioned above, that the previous outbreaks also showed similar high velocities, high luminosity, and long term variability, they propose that SN 2009ip is instead a pulsational pair-instability event and that the star may have survived the 2012 September outburst.

From these comparisons we conclude that there are similarities between SN 2010jl and these two objects. All three objects could be classified as Type IIn SNe, based on the narrow lines with profiles typical of electron scattering. It is clear that they all have very dense CSM into which the shock waves are propagating. At least for SN 2009ip there is evidence for preexisting dust that may have been formed a few years before the last eruption (Foley et al. 2011; Smith et al. 2013), like SN 2010j1.

The main differences between these objects and SN 2010j1 is that the latter showed a considerably higher peak luminosity, slower decay, and an order of magnitude higher total radiated energy than did SNe 2009ip and 2010mc. We therefore believe that the basic mechanism for these objects is different from that in SN 2010jl. The fact that no very broad lines with velocities typical of core collapse SNe were seen for SN 2010jl is less important. As we have already argued, this is mainly an effect of different mass-loss rates and total mass lost.

\subsection{Putting it All Together}

In this section we summarize the main points of the previous discussion and discuss how this information can be put together into a coherent scenario for SN 2010jl.

Most of the bolometric luminosity is produced by the radiation from the radiative shock with a velocity of up to $\sim 3000 \mathrm{~km} \mathrm{~s}^{-1}$, which propagates through the dense CSM resulting from a mass-loss rate of $\gtrsim 0.1 M_{\odot} \mathrm{yr}^{-1}$ and a velocity of $\sim 100 \mathrm{~km} \mathrm{~s}^{-1}$, which may be the result of a previous LBV-like eruption. The total mass lost is $\gtrsim 3 M_{\odot}$. The ingoing X-rays from the shock will be thermalized at early epochs in the dense shell behind the shock and later in the ejecta. This will there be converted into UV and optical continuum radiation with a spectrum close to a blackbody. Most of the outgoing X-rays will be absorbed by the preshock wind and will there give rise to UV and optical emission lines.

An important issue is the relative location of the source of the UV and optical line emission and the electron scattering region. The fact that the strong high-ionization UV lines, like the N IV], C IV, and N III] lines, at early epochs had strong electron scattering wings besides the narrow component shows that at least a large fraction of these arise in or interior to the electron scattering region. The same is true for the Balmer lines. As we have already discussed in Section 4.1, the narrow lines most likely arise in a more extended region outside the denser part of the CSM. Because the velocity to which the gas is accelerated is $\propto r^{-2}$ (Equation (1)), the absence of line shifts of the narrow component is consistent with a distance $\gtrsim 2 \times 10^{16} \mathrm{~cm}$ of this (Section 4.1).

To explain the broad wings of the lines, the electron scattering optical depth of this CSM has to be $\gtrsim 3$. The mass-loss rates we find correspond to a total optical depth to electron scattering of a wind with outer radius $R_{\text {shell }}$

$$
\begin{aligned}
\tau_{\mathrm{tot}}= & 1.56\left(\frac{\dot{M}}{0.1 M_{\odot} \mathrm{yr}^{-1}}\right)\left(\frac{u_{\mathrm{w}}}{100 \mathrm{~km} \mathrm{~s}^{-1}}\right)^{-1} \\
& \times\left(\frac{V_{\mathrm{s}, 320}}{3000 \mathrm{~km} \mathrm{~s}^{-1}}\right)^{-1}\left(\frac{t}{\text { years }}\right)^{-0.82}\left(1-\frac{R_{s}}{R_{\text {shell }}}\right)
\end{aligned}
$$

if completely ionized. From this it is clear that to get large enough $\tau_{\text {tot }}$ either the shock velocity has to be lower than that corresponding to the X-ray temperature, $\sim 3000 \mathrm{~km} \mathrm{~s}^{-1}$, or the mass-loss rate in these directions is higher than the average, or most likely both, as we discuss in the end of this section.

One can estimate the extent of the ionized region, assuming that most of the X-ray and EUV emission from the cooling shock is absorbed by the CSM in front of the shock and will there give rise to a Strömgren zone (see, e.g., Fransson 1984 for a similar situation). We also assume that the X-rays emitted inward are thermalized by the cool shell and ejecta, resulting in optical and UV emission but only contributing marginally to the ionization. Balancing the number of ionizing photons, $\epsilon_{i} L / h v_{\text {ion }}$, with the number of H II recombinations, $4 \pi \alpha_{B} \int n_{e}(r)^{2} r^{2} d r$, where $\epsilon_{i}$ is the fraction of the energy going into ionizations, and with $\tau_{e}=\sigma_{T} \int n_{e} d r$, one obtains

$$
\tau_{e}=\frac{\sigma_{T}}{\alpha_{B}} \frac{m_{p} u_{\mathrm{w}}}{\dot{M}} \frac{\epsilon_{i} L}{h v_{\text {ion }}} .
$$

With $\alpha_{B}=2 \times 10^{-13} T_{4}^{-0.7} \mathrm{~cm}^{3} \mathrm{~s}^{-1}$ one finds

$$
\begin{aligned}
\tau_{e}= & 4.05 \epsilon_{i}\left(\frac{L}{10^{43} \mathrm{erg} \mathrm{s}^{-1}}\right) T_{4}^{0.7}\left(\frac{\dot{M}}{0.1 M_{\odot} \mathrm{yr}^{-1}}\right)^{-1} \\
& \times\left(\frac{u_{\mathrm{w}}}{100 \mathrm{~km} \mathrm{~s}^{-1}}\right) .
\end{aligned}
$$

Assuming that the X-ray/EUV luminosity is produced by the shock and given by Equation (10) (for $n=7.6$ ), we get

$$
\tau_{e}=1.9 \epsilon_{i} T_{4}^{0.7}\left(\frac{V_{\mathrm{s}, 320}}{3000 \mathrm{~km} \mathrm{~s}^{-1}}\right)^{3}\left(\frac{t}{320 \text { days }}\right)^{-0.54} .
$$

Therefore, one obtains naturally a width of the ionized zone in front of the shock of the order of unity independent of the mass-loss rate, wind velocity, or radius. This assumes that the total optical depth of the wind exceeds this value.

To estimate the conversion efficiency from X-rays to $\mathrm{H} \alpha$, as well as to determine the general UV and optical spectrum expected, we have made some exploratory calculations using CLOUDY (Ferland et al. 2013). For this we have taken a simple free-free X-ray spectrum and a density profile given by a $\rho \propto$ $r^{-2}$ wind. The X-ray temperature was $10 \mathrm{keV}$, and we studied a range of shock luminosities and mass-loss rates. A problem here is that for the most interesting parameters the electron scattering optical depth is larger than unity (Equation (17)), which cannot be handled properly by CLOUDY. As pointed out by Chevalier \& Irwin (2012), one effect of this is that the state of ionization is underestimated because the relevant quantity for this is the ratio of the radiation to matter density. This is given by $\zeta=\tau_{e} L / n r^{2}$, increasing the usual ionization parameter by a factor of $\tau_{e}$. Nevertheless, the qualitative results are interesting.

With regard to the efficiency of X-ray to $\mathrm{H} \alpha$ conversion, we find that it is very difficult to obtain an efficiency larger 
than $\sim 5 \%-7 \%$, and then only for large column densities and for densities less than $\sim 10^{10} \mathrm{~cm}^{-3}$. Above this density the efficiency decreases rapidly due to thermalization of the $\mathrm{H} \alpha$ line. The electron density we infer from the bolometric luminosity in Section 4.5 is for $n(\mathrm{He}) / n(\mathrm{H})=0.1$

$$
\begin{aligned}
n_{e}= & 2.86 \times 10^{9}\left(\frac{\dot{M}}{0.1 M_{\odot} \mathrm{yr}^{-1}}\right)\left(\frac{u_{\mathrm{w}}}{100 \mathrm{~km} \mathrm{~s}^{-1}}\right)^{-1} \\
& \times\left(\frac{r}{3 \times 10^{15} \mathrm{~cm}}\right)^{-2} \mathrm{~cm}^{-3},
\end{aligned}
$$

which is in the range where we expect a high $\mathrm{H} \alpha$ efficiency. Further, the observed maximum $\mathrm{H} \alpha$ luminosity was $\sim 1 \times$ $10^{42} \mathrm{erg} \mathrm{s}^{-1}$ (Figure 13). Although we find that there is some contribution from the photospheric emission from ionizations in the Balmer continuum, the observed $\mathrm{H} \alpha$ luminosity implies a total X-ray luminosity of $\gtrsim 10^{43} \mathrm{erg} \mathrm{s}^{-1}$. There is therefore a rough agreement between the bolometric luminosity, the $\mathrm{H} \alpha$ luminosity, and a high $\mathrm{H} \alpha$ efficiency.

Compared to this, the observed level of X-rays is $\sim 10^{42} \mathrm{erg} \mathrm{s}^{-1}$ (Chandra et al. 2012b). This is by itself no problem because most of the X-rays may be absorbed by the preshock gas, and this is in fact needed to explain the UV emission lines. A major problem is, however, that, as discussed in the Introduction, the X-rays at 59 days indicated a column density of $\sim 10^{24} \mathrm{~cm}^{-2}$, decreasing to $3 \times 10^{23} \mathrm{~cm}^{-2}$ at 373 days. ${ }^{13}$ This column density corresponds to an electron scattering depth of $\tau_{\mathrm{e}} \lesssim 1$, which is too low to explain the line profiles in the optical.

There are several possible explanations for this discrepancy. Ofek et al. (2014) argue that the column density could be decreased by a higher ionization due to a large electronscattering optical depth. As we argue above, the optical depth of the ionized gas is limited, and the effect will not be dramatic. Instead, we believe that the different column densities are due to large-scale asymmetries in the outflow, giving rise to different column densities in different directions. Alternatively, it could be due to small-scale inhomogeneities resulting in a leakage of $\mathrm{X}$-rays in the low-density regions. This is also supported by the low column density that Ofek et al. (2014) found from their $N u S T A R+X M M$ observations at 728-754 days. We discuss further evidence for these possibilities below.

For a wind, most of $\mathrm{H} \alpha$ originates close to the shock, as can be seen from $d L / d r=4 \pi \alpha_{B} r^{2} n_{e}^{2} \propto 1 / r^{2}$. This is also the region of largest optical depth to electron scattering. Emission and scattering therefore take place in the same region, although some of the $\mathrm{H} \alpha$ emission also occurs at low optical depths, resulting in a narrow line core as long as the bulk velocity of the emitting material is low (Section 4.4.3). From our CLOUDY calculations we find that high-ionization lines, like $\left.N_{\text {III }} \lambda 1750, N_{\text {IV }}\right] \lambda 1486$, and $\mathrm{C}$ IV $\lambda \lambda 1548,1551$, arise interior to the $\mathrm{H} \alpha$-emitting region and therefore experience the same electron scattering as $\mathrm{H} \alpha$. We also find that the luminosities of these lines are comparable to $\mathrm{H} \alpha$, as is observed. For constant X-ray luminosity, the $\mathrm{H} \alpha / \mathrm{H} \beta$

\footnotetext{
13 Chandra et al. used a metallicity of 0.3 times solar in agreement with that derived for the host galaxy. The connection between the hydrogen column density and the X-ray absorption, which is dominated by metals, depends on the metallicity. The main indication of synthesized material in the outer layers is the CNO processing discussed in Section 3.6. This only results in a redistribution between the $\mathrm{CNO}$ elements, which have similar cross sections and ionization thresholds. As discussed later, we do not find any other indication of processing. It is therefore reasonable to assume that, except for a different host metallicity, the standard conversion between X-ray absorption column density and $N_{\mathrm{H}}$, and therefore electron scattering depth, applies.
}

ratio increases as the density decreases, which may explain the trend seen in Figure 13. The temperature in the $\mathrm{H} \alpha$-emitting zone is $\sim(1.5-2) \times 10^{4} \mathrm{~K}$.

A related model is discussed by Dessart et al. (2009), who calculate the line formation in Type IIn SNe with application to SN 1994W. Although the authors point out that this is only a preliminary attempt and related to a specific scenario, this is the only self-consistent modeling of the line formation we are aware of. The density of the envelope in the model by Dessart et al. (2009) is $3 \times 10^{9} \mathrm{~cm}^{-3}$, which is similar to what we find for the density in front of the shock. Dessart et al. also point out a number of useful signatures, depending on the relative location of the region where the Balmer emission originates and the electron scattering region. In particular, they find that if the photons are internally generated, $\mathrm{H} \alpha, \mathrm{H} \beta$, and $\mathrm{H} \gamma$ form at increasing optical depth. The electron scattering depth also increases for these lines, leading to increasingly strong wings for $\mathrm{H} \beta$ and $\mathrm{H} \gamma$ compared to $\mathrm{H} \alpha$. If the lines, on the other hand, are created outside the scattering region, they are expected to have similar line profiles.

In principle, these possibilities can be tested with our observations. The limited $\mathrm{S} / \mathrm{N}$ and relatively steep Balmer decrement makes it difficult to see any differences between these line profiles. The best set of line profiles are from our spectrum from $\mathrm{X}$-shooter in Figure 31. This does show some minor differences in the width of the lines, but these are sensitive to the exact way the continuum is subtracted as well as to blending by other lines. The latter is especially important for the $\mathrm{He}_{\mathrm{I}} \lambda 10,830$ line, which is blended with $\mathrm{Pa} \gamma$; the red wing of $\mathrm{H} \beta$ also shows indications of blending. It is therefore premature to draw any firm conclusions.

As argued in Section 4.4.3, we believe that the bulk velocity of $\sim 600-700 \mathrm{~km} \mathrm{~s}^{-1}$ of the Balmer emitting region is a result of the preacceleration of the circumstellar material in front of the main shock by the intense radiation from the thermalized radiation from the shock. This is supported by the agreement between the observed velocity evolution and that predicted from the evolution of the bolometric luminosity. A surprising result is that, to produce the line shift of the $\mathrm{H} \alpha$ line, we required that most of the emitting material was moving with similar velocity relative to us. This in turn requires the flow to subtend a fairly small solid angle. The expansion does, however, not necessarily have to be in our LOS, which would increase the true expansion velocity by $1 / \cos \theta$, where $\theta$ is the angle between these directions.

Both the line shifts and the X-ray observations therefore give evidence for a highly asymmetric ejecta interaction in SN 2010j1. On a larger scale we find evidence for an asymmetric dust distribution (Section 4.2). In addition, the early polarization measurements by Patat et al. (2011) at 14.7 days after discovery showed a large, constant continuum polarization of $1.2 \%-2 \%$, indicating an asphericity with axial ratio $\sim 0.7$. Close to the center of $\mathrm{H} \alpha$ the polarization decreased to a low level. The wings of the line have, however, a polarization close to the continuum level. As suggested by the authors, this is probably an indication of another component dominated by recombination. Our high-resolution observations do show that, especially at early phases, the narrow component from the CSM is strong and will therefore dominate in the line center. Most likely this is produced mainly by recombination and is probably also coming from a more symmetric CSM, as indicated by the strong absorption component of the P-Cygni lines (Section 4.1). 
Asymmetries have been discussed from time to time for Type IIn SNe. This includes the Type IIn SN 1988Z (Chugai \& Danziger 1994), SN 1995N (Fransson et al. 2002), SN 1998S (Leonard et al. 2000; Fransson et al. 2005), and SN 2006jd (Stritzinger et al. 2012). In these cases it has been mainly disklike or clumpy distributions that have been considered. A clumpy model does, however, not have the large-scale asymmetry needed to match the observed bulk velocity of hydrogen lines. A disk-like geometry also has problems because one would then expect the expansion into a disk to be cylindrically symmetric, which would not give the coherent velocity in the LOS indicated by the broad line profiles above.

Instead, we believe that a bipolar outflow may be more compatible with the observations. As was noted in Section 4.1, the expansion velocity of the molecular shell in Eta Carinae is highly anisotropic with a velocity of up to $\sim 650 \mathrm{~km} \mathrm{~s}^{-1}$ at the poles (Smith 2006). Even more interesting is that most of the mass is ejected between $45^{\circ}$ and the pole. Higher velocities up to $\sim 6000 \mathrm{~km} \mathrm{~s}^{-1}$ are also seen, although little mass is involved (Smith 2008).

The initially small shift of the lines in SN 2010j1, however, requires this high-density shell to have a low velocity, $\lesssim 100 \mathrm{~km} \mathrm{~s}^{-1}$, before the explosion, similar to what we see from the narrow lines. The increasing velocity shift in $\mathrm{H} \alpha$ may then be a direct representation of the gradual acceleration of this shell. The column density of the shell in Eta Carinae is $\sim 10^{24} \mathrm{~cm}^{-2}$ located at a radius between $3 \times 10^{16} \mathrm{~cm}$ and $3 \times 10^{17} \mathrm{~cm}$ (Smith 2006). This scales as $N_{\mathrm{H}} \propto r^{-2}$, so if one would observe it at an earlier stage with a radius of a few $\times 10^{15} \mathrm{~cm}$, as in SN 2010j1, the column density would be two to three orders of magnitude higher, more than sufficient for explaining the large electron-scattering optical depths we have evidence for in the broad lines.

The planar geometry of the $\mathrm{H} \alpha$-emitting gas may resemble that seen in Eta Carinae at high latitudes. Smith (2006) find above $\sim 60^{\circ}$ latitude an almost perfectly planar shape of the molecular shell, containing most of the gas (see, e.g., Figure 4 in Smith 2006). If one would observe an explosion in this direction (or higher latitude), the expansion would clearly be planar. The large column density of the shell would also prevent the observation of the rear parts of the CSM.

The scenario we infer from the observations has many qualitative similarities to the one calculated by van Marle et al. (2010). Using two-dimensional hydrodynamical simulations, they have calculated the interaction of a core collapse $\mathrm{SN}$ with a dense circumstellar shell ejected two years before explosion. The total shell mass was in most cases $10 M_{\odot}$, while the ejecta mass for this shell mass was varied between 10-60 $M_{\odot}$. Models with both spherical geometry and a density and mass distribution similar to the bipolar structure observed for Eta Carinae were calculated. Optically thin cooling, but no radiative transfer, was included. At the time the shock collides with the shell, the shock velocity is $\sim 5500 \mathrm{~km} \mathrm{~s}^{-1}$ for the chosen parameters, but it decreases to $1000-2000 \mathrm{~km} \mathrm{~s}^{-1}$ in the shell, depending on mass of ejecta, shell mass, and SN energy. Because of the large density and low velocity, implying a shock temperature of $\lesssim 10^{7} \mathrm{~K}$, the shock becomes radiative. After the shock has penetrated the shell, the velocity again increases, and the shock then in most cases becomes adiabatic. This evolution illustrates what happens when an SN with "normal" energy interacts with a dense CSM. The low shock velocity resulting from this is in line with what is observed for $\mathrm{SN}$ $2010 j 1$.
In their bipolar simulations (models D01-D03), the ejecta first interact with the equatorial region and then later with the polar region. Because of the smaller column density in the equatorial direction, the shock penetrates this region first, at which time the shock temperature increases rapidly. In the polar direction it takes a considerably longer time for the shock to reach the outer boundary of the shell, and the shock velocity is also lower. This may illustrate the range of velocities that may be present in an anisotropic CSM, and it may explain the different column densities inferred from the X-rays and the electron scattering wings, discussed above. These anisotropic models demonstrate nicely how one can have both high shock velocities (in the equatorial directions), giving rise to hard $\mathrm{X}$-rays, and slow shocks still in the optically thick circumstellar shell (in the polar directions) at the same time. The drop in luminosity during the breakout phase in the bipolar models is $L \propto t^{-\alpha}$, where $\alpha=3.6-4.4$ (Figure 16 in van Marle et al. 2010). This depends on the parameters of the models, but it is at least in qualitative agreement with the observations, which show that $L(t) \propto t^{-3.4}$ after the break (Section 3.2).

For the spherical models in van Marle et al. (2010), the light curves in general consist of a rapid rise when the shock encounters the shell and a slowly decreasing plateau. As the shock passes the outer shell, there is a rapid drop as the shock becomes adiabatic. Because of the gradual emergence of the shock with latitude, the bipolar case results in a smoother evolution of the light curve without the same sharp break as the spherical shell. This result depends on the angular dependence of the column density. A stronger concentration to the poles would give a sharper drop of the light curve, in line with what we see in SN 2010j1. The efficiency in converting kinetic energy into radiation was found to be in the range 10-40\%, decreasing with increasing ejecta mass and expansion velocity of the shell (determining the distance of the shell from the $\mathrm{SN}$ ). With an efficiency in this range, the radiated energy of $\sim 6.5 \times 10^{50} \mathrm{erg}$ would correspond to an explosion energy of $\sim(4 \pm 3) \times 10^{51} \mathrm{erg}$.

The fact that we do not see evidence for expansion in the opposite direction is a natural result of the obscuration of this region by the optically thick material in the core of the SN and from the ionized material exterior to this moving toward us. The decline in the flux at late epochs is a result of the decrease in the photospheric radius (see Table 5), which in principle might indicate that the extent of the photosphere is not large enough to obscure the receding side of the SN. However, the photospheric radius is the thermalization radius, which must be deep inside the $\tau_{e}=1$ radius. In our last spectra, at $\sim 1100$ days the optical depth to electron scattering is likely to be $\gtrsim 1$. The obscuration of the back side of the SN by the scattering region even at late epochs is therefore not a problem. As we have already discussed, at late epochs the shock should have penetrated the dense circumstellar shell in the low column density regions close to the equator, while it is still inside the optically thick region in our LOS, although outside the photosphere.

With regard to the narrow lines, we note that a steady wind with a mass-loss rate of $10^{-3} M_{\odot} \mathrm{yr}^{-1}$, as in the quiescent state in Eta Carinae, and wind velocity of $100 \mathrm{~km} \mathrm{~s}^{-1}$ corresponds to a density of $\sim 3 \times$ $10^{6}\left(\dot{M} / 10^{-3} M_{\odot} \mathrm{yr}^{-1}\right)\left(\mathrm{u}_{\mathrm{w}} / 100 \mathrm{~km} \mathrm{~s}^{-1}\right)^{-1}\left(\mathrm{r} / 10^{16} \mathrm{~cm}\right)^{-2} \mathrm{~cm}^{-3}$, which is similar to what we find from the nebular diagnostics of the narrow lines for SN 2010jl, but below that of the Balmer lines. A larger radial extent of the narrow line region,as indicated from the ionization parameter (Section 4.1), is natural in this context. 
The deep absorption component in the P-Cygni profile of the narrow $\mathrm{H} \alpha$ line (Figure 12) means that this material must have an appreciable covering factor of the underlying emission from the SN. The emission component of this, as well as the depth of the absorption, tends to decrease with time. This may be a result of the expansion of the SN relative to the slow CSM. An example of this kind of evolution can be seen in the analytical models in Figure 5 of Fransson (1984), where the relative size of the scattering layer to the "photospheric" emission is varied.

The dust shell, giving rise to the echo, is at a considerable larger distance, $\gtrsim 10^{17} \mathrm{~cm}$. The high temperature and large distance argues for small dust grains. To reproduce the NIR light curve, the dust should have an anisotropic distribution. The dust is likely to have been formed in previous mass loss phases of the progenitor, as seen in Eta Carinae.

Finally, we remark that even in our last full spectrum at $\sim 850$ days there is no clear transition to a nebular stage, although the spectrum is dominated by emission lines, with $\mathrm{H} \alpha$ by far the strongest line, rather than continuum emission (Figure 7). Electron scattering is apparently still important in the line-emitting region, and there are no signs of any processed material in even the last spectrum. This may in principle speak in favor of the pure LBV scenario without core collapse. Because of the extreme mass-loss rate, the hydrogen envelope is, however, likely to still be opaque to the emission from the core (Section 4.6). We will therefore have to wait until the optical depth is low enough for any firm conclusions.

\subsection{Progenitor Scenarios}

As discussed by Miller et al. (2010; see also Smith et al. 2008a, 2010a), the extremely luminous Type IIn SNe, with absolute magnitude brighter than $M_{V}=-20$, may arise from ejections where the ejected shell has not expanded to more than a few $\times 10^{15} \mathrm{~cm}$, resulting in a dense, optically thick shell. This would correspond to SN 2010jl. Ejected shells that have expanded to larger radii, and therefore have lower density and optical depth, would give rise to more "normal" Type IIns, like SNe $1988 Z$ and 2008iy, with absolute magnitudes fainter than -20 . These are also expected to be strong $\mathrm{X}$-ray and radio sources, in contrast to the more luminous Type IIns. As we discuss in Section 4.6, the mass-loss rate and its duration may be the most important parameters distinguishing these properties.

Although we have in this paper mainly discussed our results in terms of conditions around LBVs, the standard LBV scenario has a number of problems in explaining the Type IIn SNe. In particular, the high frequency of Type IIns may be incompatible with very massive progenitors, and the evolutionary state of the LBVs may also be a problem. Recently there have been different suggestions, discussed below, addressing these problems. One should note that the LBV interpretation is mainly phenomenological, based on observational characteristics like the very dense CSM, the CNO processing, and the typical velocities for the CSM. It has also been suggested that a large fraction of Type IIn SNe may arise as a result of explosions in red supergiants with enhanced mass loss (Fransson et al. 2002; Smith et al. 2009), which would alleviate these problems.

A single-star version has recently been put forward by Groh et al. (2013) based on a moderately fast rotating star with a mass 20-25 $M_{\odot}$. The average rotational velocity on the main sequence was $\sim 200 \mathrm{~km} \mathrm{~s}^{-1}$ for these models. The rotation, in combination with mixing, results in a more massive He core than for a nonrotating star. This has the consequence that the star ends up as a hot supergiant, with effective temperature $\sim 2 \times 10^{4} \mathrm{~K}$ and with a thin hydrogen envelope. The heavy mass loss in the red supergiant and final hot phase results in a dense CSM.

The most interesting result in the paper by Groh et al. is that a full NLTE atmospheric calculation of the hydrostatic and wind regions in the final phase gives a spectrum similar to that of an LBV, with a large number of emission lines. Because of heavy mass loss and mixing, CNO products are transported to the surface, with essentially complete CNO burning. Their $20 M_{\odot}$ model has $\mathrm{N} / \mathrm{C}=128$ and $\mathrm{N} / \mathrm{O}=16$ at the end of $\mathrm{C}$ burning. These are considerably higher than our determination for SN 2010jl, including the uncertainty in these. As discussed by Groh et al., this could perhaps be explained by He-burning products mixed to the surface. It may, however, also indicate less complete $\mathrm{CNO}$ burning, as is, e.g., seen in the $15 M_{\odot}$ models by Ekström et al. (2012). These lower mass stars, however, end their life as red supergiants, not LBV-like objects. A dense CSM may nevertheless be present, but the wind velocity would probably be lower than we observe.

The mass-loss rate Groh et al. find for the 20-25 $M_{\odot}$ models in the pre-SN phase is (1-4) $\times 10^{-4} M_{\odot} \mathrm{yr}^{-1}$ and wind velocity $270-320 \mathrm{~km} \mathrm{~s}^{-1}$. The wind velocity is considerably higher than what we find for SN 2010j1. Scaling to a lower wind velocity, this mass-loss rate corresponds to an electron density of $\sim 2.3 \times$ $10^{5}\left(\dot{M} / 10^{-4} M_{\odot} \mathrm{yr}^{-1}\right)\left(\mathrm{u}_{\mathrm{w}} / 300 \mathrm{~km} \mathrm{~s}^{-1}\right)^{-1}\left(\mathrm{r} / 10^{16} \mathrm{~cm}\right)^{-2} \mathrm{~cm}^{-3}$, where $r$ is the radius of the line-emitting material. We have assumed a $\mathrm{He} / \mathrm{H}$ ratio of 0.8 by mass (Groh et al. 2013). Depending on the mass-loss rate and radius of the line-emitting gas, this may be compatible with what we observe for the narrow lines from the CSM in SN 2010jl. However, there are large uncertainties in this estimate. First, as discussed in Sections 3.5 and 4.3 , there is considerable uncertainty in the radius of the lineemitting gas. Also, the mass-loss rate of the models is highly uncertain because, as Groh et al. (2013) remark, these stars are close to the Eddington luminosity. An increased mass loss is therefore not unexpected, as discussed by Gräfener et al. (2012, and references therein). The mass-loss rate could also have been higher in the previous red supergiant phase, further boosting the density of the CSM at large radii. Rotational effects could also make the mass loss highly anisotropic. Overall, we find that given these uncertainties there is at least some rough agreement between the models and our observations. This scenario may therefore solve the evolutionary and rate problems and at the same time at least qualitatively explain the observational LBV characteristics. A major issue is to explain the mechanism behind the outbursts needed to explain the dense inner shell.

As a second alternative, based now on a binary model, Chevalier (2012) has proposed a merger scenario, where a compact companion in the form of either a neutron star or a black hole may merge with a normal companion star. The energy released by the in-spiraling will release a large energy into the envelope of the companion star, resulting in heavy mass loss. A dense CSM will therefore result, mainly concentrated to the orbital plane of the binary. This may have implications for the line profiles, but a more definite comparison is outside the scope of this paper. We also remark that an LBV-like eruption may also be the result of a merger or possibly only a tight binary encounter in a lower mass system. This would then be in better agreement with the relatively high frequency of Type IIn SNe. The high luminosity would, however, be difficult to understand.

A third scenario is discussed by Quataert \& Shiode (2012) based on convective motions connected to the carbon burning and later stages when the core luminosity is super Eddington. This turbulence in turn excites internal gravity waves that may 
convert a fraction of their energy into sound waves. These finally dissipate their energy and cause mass loss from the stellar envelope. The advantage with this model is that it directly connects the stellar explosion and strong mass loss of the progenitor. Shiode \& Quataert (2014) have developed this further and made predictions for the duration of the wavedriven mass loss, as well as the total mass lost. Most of the mass loss occurs in the Ne- and O-burning stages, which limits the duration to $\lesssim 10$ yr before explosion. Only for stars with He-core masses below $\sim 10 M_{\odot}$, or zero-age main sequence masses below $\sim 20 M_{\odot}$, do they find substantial mass loss, with a total mass lost of $0.1-1 M_{\odot}$. Although the wind velocity we find, $\sim 100 \mathrm{~km} \mathrm{~s}^{-1}$, is close to the escape velocities of their models, the total mass lost in the wave-driven phase is substantially lower than what we find for SN 2010jl. With a duration of $\lesssim 10 \mathrm{yr}$, the extent of the dense shell, $\lesssim 3 \times 10^{15} \mathrm{~cm}$, is also on the low side. Although interesting in that this scenario directly connects the heavy mass loss phase with the explosion, many details connected to, e.g., the conversion efficiency, effects of binary interaction and are uncertain and require numerical work along the lines of Meakin \& Arnett (2006) to test this scenario. Smith \& Arnett (2014) have recently discussed the consequences of instabilities from turbulent convection in the latest burning phase, which may trigger strong mass loss just before explosion.

\section{CONCLUSIONS}

SN 2010jl represents the best-observed Type IIn supernova to date, and in addition one of the brightest. In this paper we have combined our optical, NIR, and UV observations with $\mathrm{X}$-ray observations to get a full view of this $\mathrm{SN}$ in both time and wavelength. Our most important conclusions are as follows.

1. We have presented one of the most complete data sets of any Type IIn SN, covering the UV, optical, and IR range. It also represents a nearly complete coverage in time from the explosion to $\sim 1100$ days.

2. We find a large number of narrow UV, optical, and NIR lines from the CSM from both low and very high ionization stages, including coronal lines, most likely excited by the $\mathrm{X}$-ray emission from the SN shock wave. The UV lines provide strong evidence for CNO processed gas in the CSM, with $\mathrm{N} / \mathrm{C}=25 \pm 15$ and $\mathrm{N} / \mathrm{O}=0.85 \pm 0.15$. The density of the CSM and distance to the narrow-line emission are consistent with observations of LBVs in our Galaxy.

3. The expansion velocity of the CSM makes red supergiants unlikely as progenitors, but it is consistent with LBVs.

4. The profiles of the broad lines are symmetrical in the comoving frame up to $\sim 1100$ days, with a shape typical of electron scattering, but they are shifted by an increasing velocity to $\sim 600-700 \mathrm{~km} \mathrm{~s}^{-1}$ along the LOS. The profiles of the broad lines show no strong evidence of wavelength dependence, as would be expected if dust was responsible for the shift of the broad lines. Instead, the shift of the broad lines that develop at late stages is explained as a result of the bulk velocity of the gas, rather than as a result of dust in the ejecta.

5. We find that the most likely explanation for the velocity shift is radiative acceleration by the flux from the $\mathrm{SN}$. This is a natural consequence of the large radiated total energy and slow decline over several hundred days of this $\mathrm{SN}$, resulting in a gradual acceleration, not usually seen for other SNe.
6. The optical depth needed to produce the electron scattering wings, the low and coherent bulk velocity inferred from the velocity shifts, together with the comparatively low X-ray column densities and the high X-ray temperature, provide strong evidence for an asymmetric shock wave, with both varying velocities and column density, consistent with what is inferred from polarization measurements at early epochs. A bipolar outflow from an LBV a few years before the explosion would be compatible with these observations, although we cannot exclude a one-sided asymmetry.

7. The NIR dust excess is likely to originate from an echo at a distance of $\sim 6 \times 10^{17} \mathrm{~cm}$ in the CSM of the SN. The dust is heated by the radiation from the SN to nearly the evaporation temperature. The high dust temperature and large distance may require a small grain size.

8. The expansion velocity of the CSM is $\sim 100 \mathrm{~km} \mathrm{~s}^{-1}$, and the mass-loss rate has to be $\gtrsim 0.1 M_{\odot} \mathrm{yr}^{-1}$ to explain the bolometric light curve by circumstellar interaction. The total mass lost is $\gtrsim 3 M_{\odot}$. Both the mass-loss rate and total mass lost are likely to be lower limits, depending strongly on the uncertain shock velocity and anisotropies in this.

9. There are no indications of a nebular stage, or any processed material, even at $\sim 850$ days. The last spectrum is dominated by a strong $\mathrm{H} \alpha$ line. The core region is, however, likely to still be opaque as a result of the dense CSM.

10. When compared to other Type IIn SNe, we find strong similarities with other well-observed objects, like SNe $1995 \mathrm{~N}, 1998 \mathrm{~S}, 2005 \mathrm{ip}$, and 2006jd, in terms of early electron-scattering line profiles, total radiated energy, CSM properties, and X-ray emission. The main difference is the several order of magnitude higher mass-loss rate in SN 2010j1, which slows down the evolution to the nebular stage.

11. The UV spectrum will add to the small set of nearby UVbright objects, which may dominate future high-redshift SN surveys.

We are grateful to Eran Ofek and Avishay Gal-Yam for useful comments on the draft. This research was supported by the Swedish Research Council and the National Space Board. The Oskar Klein Centre is funded by the Swedish Research Council. The CfA Supernova Program is supported by NSF grant AST-1211196 to the Harvard College Observatory and has also been supported by PHY-1125915 to the Kavli Institute of Theoretical Physics. F.B. acknowledges support from FONDECYT through Postdoctoral grant 3120227 and from the Millennium Center for Supernova Science through grant P10-064-F (funded by Programa Bicentenario de Ciencia y Tecnologa de CONICYT and Programa Iniciativa Cientf'ica Milenio de MIDEPLAN). The research of RAC is supported by NASA grant NNX12AF90G. S.B. is partially supported by the PRIN-INAF 2011 with the project Transient Universe: from ESO Large to PESSTO. Support for Program GO-12242 was provided by NASA through a grant from the Space Telescope Science Institute, which is operated by the Association of Universities for Research in Astronomy, Incorporated, under NASA contract NAS5-26555. Based on observations made with the Nordic Optical Telescope, operated by the Nordic Optical Telescope Scientific Association at the Observatorio del Roque de los Muchachos, La Palma, Spain, of the Instituto de Astrofisica de Canarias under Period P42, P43, P46, and P47 (P.I. Sollerman). The data presented here were obtained in part with ALFOSC, which is provided by the Instituto de Astrofisica de Andalucia (IAA) under a joint agreement with the University 
of Copenhagen and NOTSA, based on observations collected at the European Organisation for Astronomical Research in the Southern Hemisphere, Chile (Program 088.D-0195, P.I. Sollerman).

Facilities: HST (COS,STIS), VLT (X-shooter), FLWO:1.5 m (FAST), MMT, NOT(ALFOSC,NOTCAM), Magellan:Baade Spitzer

\section{REFERENCES}

Andrews, J. E., Clayton, G. C., Wesson, R., et al. 2011, AJ, 142, 45 Asplund, M., Grevesse, N., Sauval, A. J., \& Scott, P. 2009, ARA\&A, 47, 481 Auer, L. H., \& van Blerkom, D. 1972, ApJ, 178, 175 Benetti, S., Bufano, F., Vinko, J., et al. 2010, CBET, 2536, 1 Bessell, M., \& Murphy, S. 2012, PASP, 124, 140 Bohlin, R. C., Savage, B. D., \& Drake, J. F. 1978, ApJ, 224, 132 Borish, H. J., Huang, C., Chevalier, R. A., et al. 2014, arXiv:1406.5531 Calzetti, D., Kinney, A. L., \& Storchi-Bergmann, T. 1994, ApJ, 429, 582 Cardelli, J. A., Clayton, G. C., \& Mathis, J. S. 1989, ApJ, 345, 245 Chandra, P., Chevalier, R. A., Chugai, N., et al. 2012a, ApJ, 755, 110 Chandra, P., Chevalier, R. A., Irwin, C. M., et al. 2012b, ApJL, 750, L2 Chatzopoulos, E., Wheeler, J. C., Vinko, J., et al. 2011, ApJ, 729, 143 Chevalier, R., \& Blondin, J. M. 1995, ApJ, 444, 312

Chevalier, R. A. 1976, ApJ, 207, 872

Chevalier, R. A. 2012, ApJL, 752, L2

Chevalier, R. A., \& Irwin, C. M. 2011, ApJL, 729, L6

Chevalier, R. A., \& Irwin, C. M. 2012, ApJL, 747, L17

Chugai, N. N. 2001, MNRAS, 326, 1448

Chugai, N. N., \& Danziger, I. J. 1994, MNRAS, 268, 173

Crawford, F. L., Keenan, F. P., Aggarwal, K. M., et al. 2000, A\&A, 362, 730

Danforth, C. W., Keeney, B. A., Stocke, J. T., Shull, J. M., \& Yao, Y. 2010, ApJ, 720,976

Dessart, L., Hillier, D. J., Gezari, S., Basa, S., \& Matheson, T. 2009, MNRAS, 394, 21

Doi, M., Tanaka, M., Fukugita, M., et al. 2010, AJ, 139, 1628

Draine, B. T. 2011, in Physics of the Interstellar and Intergalactic Medium, ed. B. T. Draine (Princeton, NJ: Princeton Univ. Press)

Draine, B. T., \& Lee, H. M. 1984, ApJ, 285, 89

Draine, B. T., \& Salpeter, E. E. 1979, ApJ, 231, 438

Dufour, R. J., Glover, T. W., Hester, J. J., et al. 1997, in ASP Conf. Ser. 120 Luminous Blue Variables: Massive Stars in Transition, ed. A. Nota \& H. Lamers (San Francisco, CA: ASP), 255

Ekström, S., Georgy, C., Eggenberger, P., et al. 2012, A\&A, 537, A146 Emmering, R. T., \& Chevalier, R. A. 1988, AJ, 95, 152

Ergon, M., Sollerman, J., Fraser, M., et al. 2014, A\&A, 562, A17

Fabricant, D., Cheimets, P., Caldwell, N., \& Geary, J. 1998, PASP, 110, 79

Falk, S. W., \& Arnett, W. D. 1977, ApJS, 33, 515

Fassia, A., Meikle, W. P. S., Chugai, N., et al. 2001, MNRAS, 325, 907

Fassia, A., Meikle, W. P. S., Vacca, W. D., et al. 2000, MNRAS, 318, 1093

Ferland, G. J., Porter, R. L., van Hoof, P. A. M., et al. 2013, RMxAA, 49, 137

Foley, R. J., Berger, E., Fox, O., et al. 2011, ApJ, 732, 32

Fox, O., Skrutskie, M. F., Chevalier, R. A., et al. 2009, ApJ, 691, 650

Fox, O. D., Chevalier, R. A., Dwek, E., et al. 2010, ApJ, 725, 1768

Fox, O. D., Chevalier, R. A., Skrutskie, M. F., et al. 2011, ApJ, 741, 7

Fox, O. D., Filippenko, A. V., Skrutskie, M. F., et al. 2013, AJ, 146, 2

Fransson, C. 1982, A\&A, 111, 140

Fransson, C. 1984, A\&A, 132, 115

Fransson, C., Challis, P. M., Chevalier, R. A., et al. 2005, ApJ, 622, 991

Fransson, C., \& Chevalier, R. A. 1989, ApJ, 343, 323

Fransson, C., Chevalier, R. A., Filippenko, A. V., et al. 2002, ApJ, 572, 350

Fransson, C., Lundqvist, P., \& Chevalier, R. A. 1996, ApJ, 461, 993

Fraser, M., Inserra, C., Jerkstrand, A., et al. 2013, MNRAS, 433, 1312

Friedman, A. S., Wood-Vasey, W. M., Marion, G. H.., et al. 2014, arXiv: 1408.0465

Gal-Yam, A. 2012, Sci, 337, 927

Gal-Yam, A., \& Leonard, D. C. 2009, Natur, 458, 865

Gal-Yam, A., Mazzali, P., Ofek, E. O., et al. 2009, Natur, 462, 624

Gall, C., Hjorth, J., Watson, D., et al. 2014, Natur, 511, 326 (G14)

Gerardy, C. L., Fesen, R. A., Nomoto, K., et al. 2002, ApJ, 575, 1007

Ginzburg, S., \& Balberg, S. 2012, ApJ, 757, 178

Goldoni, P., Royer, F., François, P., et al. 2006, Proc. SPIE, 6269, 62692K

Gorecki, A., \& Wilczewski, W. 1984, AcA, 34, 141

Gräfener, G., Owocki, S. P., \& Vink, J. S. 2012, A\&A, 538, A40

Grandi, S. A. 1980, ApJ, 238, 10

Grasberg, E. K., \& Nadyozhin, D. K. 1987, AZh, 64, 1199
Green, J. C., Froning, C. S., Osterman, S., et al. 2012, ApJ, 744, 60

Groh, J. H., Hillier, D. J., Damineli, A., et al. 2009, ApJ, 698, 1698

Groh, J. H., Meynet, G., \& Ekström, S. 2013, A\&A, 550, L7

Hicken, M., Challis, P., Kirshner, R. P., et al. 2012, ApJS, 200, 12

Hicken, M., Garnavich, P. M., Prieto, J. L., et al. 2007, ApJL, 669, L17

Hillier, D. J. 1991, A\&A, 247, 455

Hillier, D. J., Davidson, K., Ishibashi, K., \& Gull, T. 2001, ApJ, 553, 837

Hoffman, J. L., Leonard, D. C., Chornock, R., et al. 2008, ApJ, 688, 1186

Immler, S., Milne, P., \& Pooley, D. 2010, ATel, 3012, 1

Jura, M., \& Kleinmann, S. G. 1990, ApJS, 73, 769

Kallman, T. R., \& McCray, R. 1982, ApJS, 50, 263

Kasen, D., \& Bildsten, L. 2010, ApJ, 717, 245

Keenan, F. P., Aller, L. H., Hyung, S., Conlon, E. S., \& Warren, G. A. 1993, ApJ, 410, 430

Keenan, F. P., Aller, L. H., Ryans, R. S. I., \& Hyung, S. 2001, PNAS, 98, 9476

Keenan, F. P., Dufton, P. L., Feibelman, W. A., et al. 1994, ApJ, 423, 882

Keenan, F. P., Ramsbottom, C. A., Bell, K. L., et al. 1995, ApJ, 438, 500

Kiewe, M., Gal-Yam, A., Arcavi, I., et al. 2012, ApJ, 744, 10

Kochanek, C. S., Szczygiel, D. M., \& Stanek, K. Z. 2011, ApJ, 737, 76

Kotak, R., Meikle, W. P. S., Farrah, D., et al. 2009, ApJ, 704, 306

Krolik, J. H., \& McKee, C. F. 1978, ApJS, 37, 459

Kwan, J. 1984, ApJ, 283, 70

Kwan, J. 1986, ApJ, 305, 679

Landi, E., Del Zanna, G., Young, P. R., Dere, K. P., \& Mason, H. E. 2012, ApJ, 744, 99

Laor, A., \& Draine, B. T. 1993, ApJ, 402, 441

Leonard, D. C., Filippenko, A. V., Barth, A. J., \& Matheson, T. 2000, ApJ, 536,239

Lucy, L. B., Danziger, I. J., Gouiffes, C., \& Bouchet, P. 1989, in IAU Colloq. 120: Structure and Dynamics of the Interstellar Medium, ed. G. TenorioTagle, M. Moles, \& J. Melnick (Lecture Notes in Physics, Vol. 350; Berlin: Springer), 164

Maeda, K., Nozawa, T., Sahu, D. K., et al. 2013, ApJ, 776, 5

Martini, P., Stoll, R., Derwent, M. A., et al. 2011, PASP, 123, 187

Massey, P., \& Gronwall, C. 1990, ApJ, 358, 344

Matheson, T., Blondin, S., Foley, R. J., et al. 2005, AJ, 129, 2352

Matheson, T., Filippenko, A. V., Barth, A. J., et al. 2000, AJ, 120, 1487

Mathis, J. S., Rumpl, W., \& Nordsieck, K. H. 1977, ApJ, 217, 425

Mattila, S., Meikle, W. P. S., Lundqvist, P., et al. 2008, MNRAS, 389, 141

Matzner, C. D., \& McKee, C. F. 1999, ApJ, 510, 379

Mauerhan, J., \& Smith, N. 2012, MNRAS, 424, 2659

Mauerhan, J. C., Smith, N., Filippenko, A. V., et al. 2013, MNRAS, 430, 1801

Meakin, C. A., \& Arnett, D. 2006, ApJL, 637, L53

Miller, A. A., Silverman, J. M., Butler, N. R., et al. 2010, MNRAS, 404, 305

Moriya, T., Tominaga, N., Blinnikov, S. I., Baklanov, P. V., \& Sorokina, E. I. 2011, MNRAS, 415, 199

Moriya, T. J. 2014, arXiv:1403.2731

Moriya, T. J., Blinnikov, S. I., Tominaga, N., et al. 2013, MNRAS, 428, 1020

Münch, G. 1948, ApJ, 108, 116

Neill, J. D., Sullivan, M., Gal-Yam, A., et al. 2011, ApJ, 727, 15

Newton, J., \& Puckett, T. 2010, CBET, 2532, 1

Nicholl, M., Smartt, S. J., Jerkstrand, A., et al. 2013, Natur, 502, 346

Nota, A., Livio, M., Clampin, M., \& Schulte-Ladbeck, R. 1995, ApJ, 448, 788

Ofek, E. O., Sullivan, M., Cenko, S. B., et al. 2013, Natur, 494, 65

Ofek, E. O., Zoglauer, A., Boggs, S. E., et al. 2014, ApJ, 781, 42

Osterbrock, D. E., \& Ferland, G. J. 2006, in Astrophysics of Gaseous Nebulae and Active Galactic Nuclei, ed. D. E. Osterbrock \& G. J. Ferland (2nd Sausalito, CA: University Science Books)

Osterman, S., Green, J., Froning, C., et al. 2011, Ap\&SS, 335, 257

Palay, E., Nahar, S. N., Pradhan, A. K., \& Eissner, W. 2012, MNRAS, 423, L35

Pastorello, A., Cappellaro, E., Inserra, C., et al. 2013, ApJ, 767, 1

Pastorello, A., Turatto, M., Benetti, S., et al. 2002, MNRAS, 333, 27

Patat, F., Taubenberger, S., Benetti, S., Pastorello, A., \& Harutyunyan, A. 2011, A\&A, 527, L6

Pilyugin, L. S., \& Thuan, T. X. 2007, ApJ, 669, 299

Pooley, D., Lewin, W. H. G., Fox, D. W., et al. 2002, ApJ, 572, 932

Pozdniakov, L. A., Sobol, I. M., \& Siuniaev, R. A. 1977, SvA, 21, 708

Pozzo, M., Meikle, W. P. S., Fassia, A., et al. 2004, MNRAS, 352, 457

Quataert, E., \& Shiode, J. 2012, MNRAS, 423, L92

Sargent, B. A., Srinivasan, S., Meixner, M., et al. 2010, ApJ, 716, 878

Schlegel, D. J., Finkbeiner, D. P., \& Davis, M. 1998, ApJ, 500, 525

Shiode, J. H., \& Quataert, E. 2014, ApJ, 780, 96

Shirazi, M., \& Brinchmann, J. 2012, MNRAS, 421, 1043

Shull, J. M., France, K., Danforth, C. W., Smith, B., \& Tumlinson, J. 2010, ApJ, 722, 1312

Skrutskie, M. F., Cutri, R. M., Stiening, R., et al. 2006, AJ, 131, 1163

Smith, N. 2006, ApJ, 644, 1151 
Smith, N. 2008, in IAU Symp. 250, Massive Stars as Cosmic Engines ed. F. Bresolin, P. A. Crowther, \& J. Puls (Cambridge: Cambridge Univ. Press), 193

Smith, N., \& Arnett, W. D. 2014, ApJ, 785, 82

Smith, N., Cenko, S. B., Butler, N., et al. 2012a, MNRAS, 420, 1135

Smith, N., Chornock, R., Li, W., et al. 2008a, ApJ, 686, 467

Smith, N., Chornock, R., Silverman, J. M., Filippenko, A. V., \& Foley, R. J. 2010a, ApJ, 709, 856

Smith, N., Foley, R. J., \& Filippenko, A. V. 2008b, ApJ, 680, 568

Smith, N., Li, W., Foley, R. J., et al. 2007, ApJ, 666, 1116

Smith, N., Li, W., Miller, A. A., et al. 2011a, ApJ, 732, 63

Smith, N., Li, W., Silverman, J. M., Ganeshalingam, M., \& Filippenko, A. V. 2011b, MNRAS, 415, 773

Smith, N., Mauerhan, J. C., Kasliwal, M. M., \& Burgasser, A. J. 2013, MNRAS, 434, 2721

Smith, N., \& McCray, R. 2007, ApJL, 671, L17
Smith, N., Miller, A., Li, W., et al. 2010b, AJ, 139, 1451

Smith, N., \& Morse, J. A. 2004, ApJ, 605, 854

Smith, N., \& Owocki, S. P. 2006, ApJL, 645, L45

Smith, N., Silverman, J. M., Chornock, R., et al. 2009, ApJ, 695, 1334

Smith, N., Silverman, J. M., Filippenko, A. V., et al. 2012b, AJ, 143, 17

Stoll, R., Prieto, J. L., Stanek, K. Z., et al. 2011, ApJ, 730, 34

Stone, R. P. S. 1977, ApJ, 218, 767

Stritzinger, M., Hamuy, M., Suntzeff, N. B., et al. 2002, AJ, 124, 2100

Stritzinger, M., Taddia, F., Fransson, C., et al. 2012, ApJ, 756, 173

Umeda, H., \& Nomoto, K. 2008, ApJ, 673, 1014

van Marle, A. J., Smith, N., Owocki, S. P., \& van Veelen, B. 2010, MNRAS, 407, 2305

Verner, E., Bruhweiler, F., \& Gull, T. 2005, ApJ, 624, 973

Voors, R. H. M., Waters, L. B. F. M., de Koter, A., et al. 2000, A\&A, 356, 501

Weaver, T. A. 1976, ApJS, 32, 233

Zhang, T., Wang, X., Wu, C., et al. 2012, AJ, 144, 131 